Pacific

Journal of

Mathematics

UNITARY REPRESENTATIONS OF THE EXTENDED AFFINE LIE ALGEBRA $\mathfrak{g l}_{3}\left(\mathbb{C}_{q}\right)$

ZITING ZENG

Volume 233 No. 2

December 2007 


\title{
UNITARY REPRESENTATIONS OF THE EXTENDED AFFINE LIE ALGEBRA $\widehat{\mathfrak{g l}_{3}\left(\mathbb{C}_{q}\right)}$
}

\author{
ZITING ZENG
}

\begin{abstract}
We present modules for the extended affine Lie algebra $\widetilde{\mathfrak{g l}_{3}\left(\mathbb{C}_{q}\right)}$ by using the idea of free fields. We give a necessary and sufficient condition for the modules to be unitary.
\end{abstract}

\section{Introduction}

Extended affine Lie algebras are a higher dimensional generalization of affine Kac-Moody Lie algebras introduced by Høegh-Krohn and Torrésani [1990] and systematically studied in [Allison et al. 1997; Berman et al. 1996]. It turns out that any extended affine Lie algebra of type $A$ can be coordinatized by a quantum torus (or a nonassociative torus for some small rank cases). Representations for extended affine Lie algebras coordinatized by quantum tori and Lie algebras related to quantum tori have been studied in [Jakobsen and Kac 1989; Berman and Szmigielski 1999; Gao 2000a; 2000b; 2002; Eswara Rao 2004; 2003; Eswara Rao and Batra 2002; Gao and Zeng 2006; Eswara Rao and Zhao 2004; Lin and Tan 2004; 2006; Golenishcheva-Kutuzova and Kac 1998; Varagnolo and Vasserot 1998; Miki 2004; Zhang and Zhao 1996; Billig and Zhao 2004; Su and Zhu 2005; Lau 2005; Baranovsky et al. 2000] and elsewhere.

Wakimoto's free fields construction [1986] provides a remarkable way to realize affine Kac-Moody Lie algebras; see also [Fer̆gin and Frenkel 1990; Etingof et al. 1998]. In [Gao and Zeng 2006], we used Wakimoto's idea to construct a class of representations for $\widehat{\mathfrak{g l}_{2}\left(\mathbb{C}_{q}\right)}$ and found the necessary and sufficient condition for the representations to be unitary. Here, we will continue to construct representations

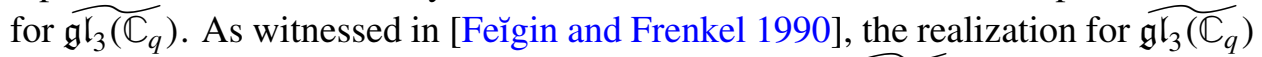

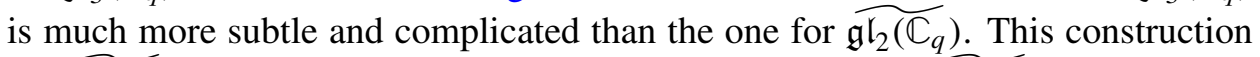
for $\widehat{\mathfrak{g l}_{3}\left(\mathbb{C}_{q}\right)}$ might shed light on the general construction for $\mathfrak{g l}_{n}\left(\mathbb{C}_{q}\right)$ with $n \geq 4$.

We then construct a hermitian form and determine when the form is positive definite (so the representations are unitary). Unlike [Gao and Zeng 2006], in which we defined the form on the monomial basis for the module (this idea goes back to [Wakimoto 1985]), we define the form directly on the basis consisting of certain

MSC2000: primary 06B15; secondary 81R50.

Keywords: extended affine Lie algebra, hermitian form, unitary representation. 
iterated module actions on a "highest weight vector" 1 . This facilitates verifying that the defined form is hermitian.

Let $q$ be a nonzero complex number. A quantum 2-torus associated to $q$ [Manin $1991]$ is the unital associative $\mathbb{C}$-algebra $\mathbb{C}_{q}\left[s^{ \pm 1}, t^{ \pm 1}\right]$ (or, simply $\mathbb{C}_{q}$ ) with generators $s^{ \pm 1}, t^{ \pm 1}$ and relations

$$
s s^{-1}=s^{-1} s=t t^{-1}=t^{-1} t=1 \text { and } t s=q s t .
$$

Define a $\mathbb{C}$-linear function $\kappa: \mathbb{C}_{q} \rightarrow \mathbb{C}$ by

$$
\kappa\left(s^{m} t^{n}\right)=\delta_{(m, n),(0,0)} .
$$

Let $d_{s}, d_{t}$ be the degree operators on $\mathbb{C}_{q}$ defined for $m, n \in \mathbb{Z}$ by

$$
d_{s}\left(s^{m} t^{n}\right)=m s^{m} t^{n}, d_{t}\left(s^{m} t^{n}\right)=n s^{m} t^{n} .
$$

Let $\mathfrak{g l}_{3}\left(\mathbb{C}_{q}\right)$ be the Lie algebra of 3 by 3 matrices with entries in $\mathbb{C}_{q}$. We form a natural central extension of $\mathfrak{g l}_{3}\left(\mathbb{C}_{q}\right)$ as

$$
\widehat{\mathfrak{g l}_{3}\left(\mathbb{C}_{q}\right)}=\mathfrak{g l}_{3}\left(\mathbb{C}_{q}\right) \oplus \mathbb{C} c_{s} \oplus \mathbb{C} c_{t}
$$

with Lie bracket

$$
\begin{aligned}
& {\left[E_{i j}\left(s^{m_{1}} t^{n_{1}}\right), E_{k l}\left(s^{m_{2}} t^{n_{2}}\right)\right]} \\
& \quad=\delta_{j k} q^{n_{1} m_{2}} E_{i l}\left(s^{m_{1}+m_{2}} t^{n_{1}+n_{2}}\right)-\delta_{i l} q^{n_{2} m_{1}} E_{k j}\left(s^{m_{1}+m_{2}} t^{n_{1}+n_{2}}\right) \\
& \quad+m_{1} q^{n_{1} m_{2}} \delta_{j k} \delta_{i l} \delta_{m_{1}+m_{2}, 0} \delta_{n_{1}+n_{2}, 0} c_{s}+n_{1} q^{n_{1} m_{2}} \delta_{j k} \delta_{i l} \delta_{m_{1}+m_{2}, 0} \delta_{n_{1}+n_{2}, 0} c_{t}
\end{aligned}
$$

for $m_{1}, m_{2}, n_{1}, n_{2} \in \mathbb{Z}$ and $1 \leq i, j, k, l \leq 3$, where $E_{i j}\left(s^{m} t^{n}\right)$ is the matrix whose only nonzero entry is $s^{m} t^{n}$ at the $(i, j)$ position, and $c_{s}$ and $c_{t}$ are central elements of $\widehat{\mathfrak{g l}_{3}\left(\mathbb{C}_{q}\right)}$.

The derivations $d_{s}$ and $d_{t}$ can be extended to derivations on $\mathfrak{g l}_{3}\left(\mathbb{C}_{q}\right)$. Now we can define the semidirect product of the Lie algebra $\widehat{\mathfrak{g l}_{3}\left(\mathbb{C}_{q}\right)}$ and those derivations:

$$
\widetilde{\mathfrak{g l}_{3}\left(\mathbb{C}_{q}\right)}=\widehat{\mathfrak{g l}_{3}\left(\mathbb{C}_{q}\right)} \oplus \mathbb{C} d_{s} \oplus \mathbb{C} d_{t} .
$$

The Lie algebra $\widetilde{\mathfrak{g l}_{3}\left(\mathbb{C}_{q}\right)}$ is an extended affine Lie algebra of type $A_{2}$ with nullity 2. See [Allison et al. 1997] and [Berman et al. 1996] for definitions.

\section{Module for $\widetilde{\mathfrak{g l}_{3}\left(\mathbb{C}_{q}\right)}$}

In this section, we use Wakimoto's idea [1985] to construct $\widetilde{\mathfrak{g l}_{3}\left(\mathbb{C}_{q}\right)}$-modules as was done in [Gao and Zeng 2006].

Let $\mathbb{K}_{1}=\{(3 m+1,3 n+1) \mid m, n \in \mathbb{Z}\}$ and $\mathbb{K}_{-1}=\{(3 m-1,3 n-1) \mid m, n \in \mathbb{Z}\}$ so that $\mathbb{K}_{-1}=-\mathbb{K}_{1}$. If $\boldsymbol{A}=(3 m+1,3 n+1) \in \mathbb{K}_{1}$, we always write $\boldsymbol{A}_{1}=m$ and 
$\boldsymbol{A}_{2}=n$, and similarly, if $\boldsymbol{B}=(3 m-1,3 n-1) \in \mathbb{K}_{-1}$, then $\boldsymbol{B}_{1}=m$ and $\boldsymbol{B}_{2}=n$. Let

$$
V=\mathbb{C}\left[x_{\boldsymbol{A}}, x_{\boldsymbol{B}}: \boldsymbol{A} \in \mathbb{K}_{1}, \boldsymbol{B} \in \mathbb{K}_{-1}\right]
$$

be the (commutative) polynomial ring of infinitely many variables. The operators $x_{(m, n)}$ and $\partial / \partial x_{(m, n)}$ act on $V$ by the usual multiplication and differentiation.

Form a family of $2 \times 2$ lower triangular matrices

$$
X_{m, n}=\left(\begin{array}{cc}
a_{(m, n)} & 0 \\
c_{(m, n)} & d_{(m, n)}
\end{array}\right) \in \mathrm{SL}_{2}(\mathbb{C})
$$

for $(m, n) \in \mathbb{K}_{1} \cup \mathbb{K}_{-1}\left(\right.$ so that $\left.a_{(m, n)} d_{(m, n)}=1\right)$. Set

$$
P_{(m, n)}=a_{(m, n)} \frac{\partial}{\partial x_{(m, n)}} \quad \text { and } \quad Q_{(m, n)}=c_{(m, n)} \frac{\partial}{\partial x_{(m, n)}}+d_{(m, n)} x_{(m, n)}
$$

for $(m, n) \in \mathbb{K}_{1} \cup \mathbb{K}_{-1}$. Then for $\boldsymbol{A}, \boldsymbol{A}^{\prime} \in \mathbb{K}_{1}$ and $\boldsymbol{B}, \boldsymbol{B}^{\prime} \in \mathbb{K}_{-1}$,

$$
\begin{aligned}
& {\left[P_{\boldsymbol{A}}, P_{\boldsymbol{A}^{\prime}}\right]=\left[Q_{\boldsymbol{A}}, Q_{\boldsymbol{A}^{\prime}}\right]=\left[P_{\boldsymbol{B}}, P_{\boldsymbol{B}^{\prime}}\right]=\left[Q_{\boldsymbol{B}}, Q_{\boldsymbol{B}^{\prime}}\right]=0} \\
& {\left[P_{\boldsymbol{A}}, P_{\boldsymbol{B}}\right]=\left[P_{\boldsymbol{A}}, Q_{\boldsymbol{B}}\right]=\left[Q_{\boldsymbol{A}}, Q_{\boldsymbol{B}}\right]=\left[P_{\boldsymbol{B}}, Q_{\boldsymbol{A}}\right]=0} \\
& {\left[P_{\boldsymbol{A}}, Q_{\boldsymbol{A}^{\prime}}\right]=\delta_{\boldsymbol{A}, \boldsymbol{A}^{\prime}}} \\
& {\left[P_{\boldsymbol{B}}, Q_{\boldsymbol{B}^{\prime}}\right]=\delta_{\boldsymbol{B}, \boldsymbol{B}^{\prime}} .}
\end{aligned}
$$

Fixing a complex number $\mu$, we define operators on $V$. For the rest of this paper, sums involving $\boldsymbol{A}$ and $\boldsymbol{A}^{\prime}$ (or any other decorated $\boldsymbol{A}$ ) will range over $\mathbb{K}_{1}$. Similarly, the $\boldsymbol{B}$ 's range over $\mathbb{K}_{-1}$. That is, we write $\sum_{\boldsymbol{A} \in \mathbb{K}_{1}}$ simply as $\sum_{\boldsymbol{A}}$, and so on.

$$
\begin{aligned}
& e_{21}^{(\mu)}\left(m_{1}, n_{1}\right)=-q^{-m_{1} n_{1}} \mu P_{-\left(3 m_{1}-1,3 n_{1}-1\right)} \\
& -\sum_{\boldsymbol{A}, \boldsymbol{A}^{\prime}} q^{n_{1} \boldsymbol{A}_{1}^{\prime}+\boldsymbol{A}_{2} m_{1}+\boldsymbol{A}_{2} \boldsymbol{A}_{1}^{\prime}} Q_{\boldsymbol{A}+\boldsymbol{A}^{\prime}+\left(3 m_{1}-1,3 n_{1}-1\right)} P_{\boldsymbol{A}} P_{\boldsymbol{A}^{\prime}} \\
& \quad-\sum_{\boldsymbol{A}, \boldsymbol{B}} q^{n_{1} \boldsymbol{A}_{1}+\boldsymbol{B}_{2} m_{1}+\boldsymbol{B}_{2} \boldsymbol{A}_{1}} Q_{\boldsymbol{A}+\boldsymbol{B}+\left(3 m_{1}-1,3 n_{1}-1\right)} P_{\boldsymbol{A}} P_{\boldsymbol{B}},
\end{aligned}
$$

$$
\begin{aligned}
e_{12}^{(\mu)}\left(m_{1}, n_{1}\right) & =Q_{\left(3 m_{1}+1,3 n_{1}+1\right)}, \\
e_{11}^{(\mu)}\left(m_{1}, n_{1}\right) & =\frac{1}{2} \mu \delta_{\left(m_{1}, n_{1}\right),(0,0)}+\sum_{\boldsymbol{A}} q^{\boldsymbol{A}_{1} n_{1}} Q_{\boldsymbol{A}+\left(3 m_{1}, 3 n_{1}\right)} P_{\boldsymbol{A}}, \\
e_{22}^{(\mu)}\left(m_{1}, n_{1}\right) & =-\frac{1}{2} \mu \delta_{\left(m_{1}, n_{1}\right),(0,0)} \\
& -\sum_{\boldsymbol{A}} q^{\boldsymbol{A}_{2} m_{1}} Q_{\boldsymbol{A}+\left(3 m_{1}, 3 n_{1}\right)} P_{\boldsymbol{A}}-\sum_{\boldsymbol{B}} q^{\boldsymbol{B}_{2} m_{1}} Q_{\boldsymbol{B}+\left(3 m_{1}, 3 n_{1}\right)} P_{\boldsymbol{B}}, \\
e_{23}^{(\mu)}\left(m_{1}, n_{1}\right) & =-q^{-m_{1} n_{1}} \mu P_{-\left(3 m_{1}+1,3 n_{1}+1\right)}
\end{aligned}
$$$$
\begin{aligned}
- & \sum_{\boldsymbol{A}, \boldsymbol{B}} q^{n_{1} \boldsymbol{B}_{1}+\boldsymbol{A}_{2} m_{1}+\boldsymbol{A}_{2} \boldsymbol{B}_{1}} Q_{\boldsymbol{A}+\boldsymbol{B}+\left(3 m_{1}+1,3 n_{1}+1\right)} P_{\boldsymbol{A}} P_{\boldsymbol{B}} \\
& -\sum_{\boldsymbol{B}, \boldsymbol{B}^{\prime}} q^{n_{1} \boldsymbol{B}_{1}^{\prime}+\boldsymbol{B}_{2} m_{1}+\boldsymbol{B}_{2} \boldsymbol{B}_{1}^{\prime}} Q_{\boldsymbol{B}+\boldsymbol{B}^{\prime}+\left(3 m_{1}+1,3 n_{1}+1\right)} P_{\boldsymbol{B}} P_{\boldsymbol{B}^{\prime}}
\end{aligned}
$$ 


$$
\begin{aligned}
& e_{32}^{(\mu)}\left(m_{1}, n_{1}\right)=Q_{\left(3 m_{1}-1,3 n_{1}-1\right)}, \\
& e_{31}^{(\mu)}\left(m_{1}, n_{1}\right)=\sum_{\boldsymbol{A}} q^{\boldsymbol{A}_{1} n_{1}} Q_{\boldsymbol{A}+\left(3 m_{1}-2,3 n_{1}-2\right)} P_{\boldsymbol{A}}, \\
& e_{13}^{(\mu)}\left(m_{1}, n_{1}\right)=\sum_{\boldsymbol{B}} q^{\boldsymbol{B}_{1} n_{1}} Q_{\boldsymbol{B}+\left(3 m_{1}+2,3 n_{1}+2\right)} P_{\boldsymbol{B}}, \\
& e_{33}^{(\mu)}\left(m_{1}, n_{1}\right)=\frac{1}{2} \mu \delta_{\left(m_{1}, n_{1}\right),(0,0)}+\sum_{\boldsymbol{B}} q^{\boldsymbol{B}_{1} n_{1}} Q_{\boldsymbol{B}+\left(3 m_{1}, 3 n_{1}\right)} P_{\boldsymbol{B}}, \\
& D_{1}^{(\mu)}=\sum_{\boldsymbol{A}} \boldsymbol{A}_{1} Q_{\boldsymbol{A}} P_{\boldsymbol{A}}+\sum_{\boldsymbol{B}} \boldsymbol{B}_{1} Q_{\boldsymbol{B}} P_{\boldsymbol{B}}, \\
& D_{2}^{(\mu)}=\sum_{\boldsymbol{A}} \boldsymbol{A}_{2} Q_{\boldsymbol{A}} P_{\boldsymbol{A}}+\sum_{\boldsymbol{B}} \boldsymbol{B}_{2} Q_{\boldsymbol{B}} P_{\boldsymbol{B}} .
\end{aligned}
$$

Although the operators are infinite sums, they are well defined as operators on $V$. Now we have the following result:

Theorem 2.1. The linear map $\pi: \widetilde{\mathfrak{g l}_{3}\left(\mathbb{C}_{q}\right)} \rightarrow$ End $V$ given by

$$
\begin{array}{lll}
\pi\left(E_{i j}\left(s^{m_{1}} t^{n_{1}}\right)\right)=e^{(\mu)}{ }_{i j}\left(m_{1}, n_{1}\right), & \pi\left(d_{s}\right)=D_{1}^{(\mu)}, & \pi\left(c_{s}\right)=0, \\
\pi\left(d_{t}\right)=D_{2}^{(\mu)}, & \pi\left(c_{t}\right)=0,
\end{array}
$$

for $m_{1}, n_{1} \in \mathbb{Z}$ and $1 \leq i, j \leq 3$ is a Lie algebra homomorphism.

Proof. The proof is straightforward. However, we will provide a few details. It suffices to check the Lie bracket (1-1). We will do this systematically so that we won't miss any cases.

First, we have

$$
\begin{aligned}
{\left[e^{(\mu)}\right.} & \left.11\left(m_{1}, n_{1}\right), e^{(\mu)}{ }_{11}\left(m_{2}, n_{2}\right)\right] \\
= & {\left[\sum_{\boldsymbol{A}} q^{\boldsymbol{A}_{1} n_{1}} Q_{\left(3 m_{1}, 3 n_{1}\right)+\boldsymbol{A}} P_{\boldsymbol{A}}, \sum_{\boldsymbol{A}^{\prime}} q^{\boldsymbol{A}_{1}^{\prime} n_{1}} Q_{\left(3 m_{2}, 3 n_{2}\right)+\boldsymbol{A}^{\prime}} P_{\boldsymbol{A}^{\prime}}\right] } \\
= & \sum_{\boldsymbol{A}^{\prime}} q^{\left(m_{2}+\boldsymbol{A}_{1}^{\prime}\right) n_{1}+\boldsymbol{A}^{\prime} n_{2}} Q_{\left(3 m_{1}, 3 n_{1}\right)+\left(3 m_{2}+3 n_{2}\right)+\boldsymbol{A}^{\prime}} P_{\boldsymbol{A}^{\prime}}+\frac{1}{2} q^{m_{2} n_{1}} \mu \delta_{\left(m_{1}+m_{2}, n_{1}+n_{2}\right),(0,0)} \\
& \quad-\sum_{\boldsymbol{A}} q^{\boldsymbol{A}_{1} n_{1}+\left(m_{1}+\boldsymbol{A}_{1}\right) n_{2}} q_{\left(3 m_{1}, 3 n_{1}\right)+\left(3 m_{2}, 3 n_{2}\right)+\boldsymbol{A}} P_{\boldsymbol{A}}-\frac{1}{2} q^{m_{1} n_{2}} \mu \delta_{\left(m_{1}+m_{2}, n_{1}+n_{2}\right),(0,0)} \\
= & q^{m_{2} n_{1}} e^{(\mu)}{ }_{11}\left(m_{1}+m_{2}, n_{1}+n_{2}\right)-q^{m_{1} n_{2}} e^{(\mu)}{ }_{11}\left(m_{1}+m_{2}, n_{1}+n_{2}\right) .
\end{aligned}
$$

The next two brackets are easy.

$$
\begin{aligned}
& {\left[e^{(\mu)}{ }_{11}\left(m_{1}, n_{1}\right), e^{(\mu)}{ }_{12}\left(m_{2}, n_{2}\right)\right]=q^{m_{2} n_{1}} e^{(\mu)}{ }_{12}\left(m_{1}+m_{2}, n_{1}+n_{2}\right),} \\
& {\left[e^{(\mu)}{ }_{11}\left(m_{1}, n_{1}\right), e^{(\mu)}{ }_{13}\left(m_{2}, n_{2}\right)\right]=q^{m_{2} n_{1}} e^{(\mu)}{ }_{13}\left(m_{1}+m_{2}, n_{1}+n_{2}\right) .}
\end{aligned}
$$


UNITARY REPRESENTATIONS OF THE EXTENDED AFFINE LIE ALGEBRA $\widetilde{\mathfrak{g l}_{3}\left(\mathbb{C}_{q}\right)} 485$

Next,

$$
\begin{aligned}
& {\left[e^{(\mu)}{ }_{11}\left(m_{1}, n_{1}\right), e^{(\mu)}{ }_{21}\left(m_{2}, n_{2}\right)\right]} \\
& =\sum_{\boldsymbol{A}}(-\mu) q^{\boldsymbol{A}_{1} n_{1}-m_{2} n_{2}}\left[Q_{\boldsymbol{A}+\left(3 m_{1}, 3 n_{1}\right)} P_{\boldsymbol{A}}, P_{-\left(3 m_{2}-1,3 n_{2}-1\right)}\right] \\
& -\sum_{\boldsymbol{A}, \overline{\boldsymbol{A}}, \overline{\boldsymbol{A}}^{\prime}} q^{\boldsymbol{A}_{1} n_{1}+\overline{\boldsymbol{A}}_{1}^{\prime} n_{2}+\overline{\boldsymbol{A}}_{2} m_{2}+\overline{\boldsymbol{A}}_{2} \overline{\boldsymbol{A}}_{1}^{\prime}}\left[Q_{\boldsymbol{A}+\left(3 m_{1}, 3 n_{1}\right)} P_{\boldsymbol{A}}, Q_{\overline{\boldsymbol{A}}+\overline{\boldsymbol{A}}^{\prime}+\left(3 m_{2}-1,3 n_{2}-1\right)} P_{\overline{\boldsymbol{A}}} P_{\overline{\boldsymbol{A}}^{\prime}}\right] \\
& -\sum_{\boldsymbol{A}, \overline{\boldsymbol{A}}, \boldsymbol{B}} q^{\boldsymbol{A}_{1} n_{1}+n_{2} \overline{\boldsymbol{A}}_{1}+\boldsymbol{B}_{2} m_{2}+\boldsymbol{B}_{2} \overline{\boldsymbol{A}}_{1}}\left[Q_{\boldsymbol{A}+\left(3 m_{1}, 3 n_{1}\right)} P_{\boldsymbol{A}}, Q_{\overline{\boldsymbol{A}}+\boldsymbol{B}+\left(3 m_{2}-1,3 n_{2}-1\right)} P_{\overline{\boldsymbol{A}}} P_{\boldsymbol{B}}\right] \\
& =\mu q^{-\left(m_{1}+m_{2}\right)\left(n_{1}+n_{2}\right)+m_{1} n_{2}} P_{-\left(3\left(m_{1}+m_{2}\right)-1,3\left(n_{1}+n_{2}\right)-1\right)} \\
& -\sum_{\overline{\boldsymbol{A}}, \overline{\boldsymbol{A}}^{\prime}} q^{\left(\overline{\boldsymbol{A}}_{1}+\overline{\boldsymbol{A}}_{1}^{\prime}+m_{2}\right) n_{1}+n_{2} \overline{\boldsymbol{A}}_{1}^{\prime}+\overline{\boldsymbol{A}}_{2} m_{2}+\overline{\boldsymbol{A}}_{2} \overline{\boldsymbol{A}}_{1}^{\prime}} Q_{\left(3 m_{1}, 3 n_{1}\right)+\overline{\boldsymbol{A}}+\overline{\boldsymbol{A}}^{\prime}+\left(3 m_{2}-1,3 n_{2}-1\right)} P_{\overline{\boldsymbol{A}}} P_{\overline{\boldsymbol{A}}^{\prime}} \\
& +\sum_{\boldsymbol{A}, \overline{\boldsymbol{A}}} q^{\boldsymbol{A}_{1} n_{1}+n_{2}\left(m_{1}+\boldsymbol{A}_{1}\right)+\overline{\boldsymbol{A}}_{2} m_{2}+\overline{\boldsymbol{A}}_{2}\left(m_{1}+\boldsymbol{A}_{1}\right)} Q_{\overline{\boldsymbol{A}}+\left(3 m_{1}, 3 n_{1}\right)+\boldsymbol{A}+\left(3 m_{2}-1,3 n_{2}-1\right)} P_{\overline{\boldsymbol{A}}} P_{\boldsymbol{A}} \\
& +\sum_{\boldsymbol{A}, \overline{\boldsymbol{A}}^{\prime}} q^{\boldsymbol{A}_{1} n_{1}+n_{2} \boldsymbol{A}_{1}^{\prime}+\left(n_{1}+\boldsymbol{A}_{2}\right) m_{2}+\left(n_{1}+\boldsymbol{A}_{2}\right) \overline{\boldsymbol{A}}_{1}^{\prime}} Q_{\boldsymbol{A}+\left(3 m_{1}, 3 n_{1}\right)+\overline{\boldsymbol{A}}^{\prime}+\left(3 m_{2}-1,3 n_{2}-1\right)} P_{\overline{\boldsymbol{A}}^{\prime}} P_{\boldsymbol{A}} \\
& +\sum_{\boldsymbol{A}, \boldsymbol{B}} q^{\boldsymbol{A}_{1} n_{1}+n_{2}\left(m_{1}+\boldsymbol{A}_{1}\right)+b d b_{2} m_{2}+\boldsymbol{B}_{2}\left(m_{1}+\boldsymbol{A}_{1}\right)} Q_{\boldsymbol{A}+\left(3 m_{1}, 3 n_{1}\right)+\boldsymbol{B}+\left(3 m_{2}-1,3 n_{2}-1\right)} P_{\boldsymbol{B}} P_{\boldsymbol{A}},
\end{aligned}
$$

(using that the second and fourth term cancel each other)

$$
\begin{aligned}
= & -q^{m_{1} n_{2}}\left(-\mu q^{-\left(m_{1}+m_{2}\right)\left(n_{1}+n_{2}\right)} P_{-\left(3\left(m_{1}+m_{2}\right)-1,3\left(n_{1}+n_{2}\right)-1\right)}\right. \\
& -\sum_{\boldsymbol{A}, \overline{\boldsymbol{A}}} q^{\boldsymbol{A}\left(n_{1}+n_{2}\right)+\overline{\boldsymbol{A}}_{2}\left(m_{1}+m_{2}\right)+\boldsymbol{A}_{1} \overline{\boldsymbol{A}}_{2}} Q_{\left(3\left(m_{1}+m_{2}\right)-1,3\left(n_{1}+n_{2}\right)-1\right)+\boldsymbol{A}+\overline{\boldsymbol{A}}} P_{\overline{\boldsymbol{A}}} P_{\boldsymbol{A}} \\
& \left.-\sum_{\boldsymbol{A}, \boldsymbol{B}} q^{\boldsymbol{A}_{1}\left(n_{1}+n_{2}\right)+\boldsymbol{B}_{2}\left(m_{1}+m_{2}\right)+\boldsymbol{B}_{2}+\boldsymbol{A}_{1}} Q_{\boldsymbol{A}+\boldsymbol{B}+\left(3\left(m_{1}+m_{2}\right)-1,3\left(n_{1}+n_{2}\right)-1\right)} P_{\boldsymbol{B}} P_{\boldsymbol{A}}\right) \\
= & -q^{m_{1} n_{2}} e^{(\mu)}{ }_{21}\left(m_{1}+m_{2}, n_{1}+n_{2}\right) .
\end{aligned}
$$

We easily verify the next seven brackets.

$$
\begin{aligned}
& {\left[e^{(\mu)}{ }_{11}\left(m_{1}, n_{1}\right), e^{(\mu)}{ }_{22}\left(m_{2}, n_{2}\right)\right]=0,} \\
& {\left[e^{(\mu)}{ }_{11}\left(m_{1}, n_{1}\right), e^{(\mu)}{ }_{23}\left(m_{2}, n_{2}\right)\right]=0,} \\
& {\left[e^{(\mu)}{ }_{11}\left(m_{1}, n_{1}\right), e^{(\mu)}{ }_{31}\left(m_{2}, n_{2}\right)\right]=-q^{m_{1} n_{2}} e^{(\mu)}{ }_{31}\left(m_{1}+m_{2}, n_{1}+n_{2}\right),} \\
& {\left[e^{(\mu)}{ }_{11}\left(m_{1}, n_{1}\right), e^{(\mu)}{ }_{32}\left(m_{2}, n_{2}\right)\right]=0,} \\
& {\left[e^{(\mu)}{ }_{11}\left(m_{1}, n_{1}\right), e^{(\mu)}{ }_{33}\left(m_{2}, n_{2}\right)\right]=0,} \\
& {\left[e^{(\mu)}{ }_{12}\left(m_{1}, n_{1}\right), e^{(\mu)}{ }_{12}\left(m_{2}, n_{2}\right)\right]=0,} \\
& {\left[e^{(\mu)}{ }_{12}\left(m_{1}, n_{1}\right), e^{(\mu)}{ }_{13}\left(m_{2}, n_{2}\right)\right]=0 .}
\end{aligned}
$$


Next we have

$$
\begin{aligned}
& {\left[e^{(\mu)}{ }_{12}\left(m_{1}, n_{1}\right), e^{(\mu)}{ }_{21}\left(m_{2}, n_{2}\right)\right] } \\
&=\mu q^{-m_{2} n_{2}} \delta_{\left(m_{1}, n_{1}\right),\left(-m_{2}, n_{2}\right)} \\
&+\sum_{\boldsymbol{A}^{\prime}} q^{n_{2} \boldsymbol{A}_{1}^{\prime}+n_{1} m_{2}+n_{1} \boldsymbol{A}_{1}^{\prime}} Q_{\left(3 m_{1}+1,3 n_{1}+1\right)+\boldsymbol{A}^{\prime}+\left(3 m_{2}-1,3 n_{2}-1\right)} P_{\boldsymbol{A}^{\prime}} \\
&+\sum_{\boldsymbol{A}} q^{n_{2} m_{1}+\boldsymbol{A}_{2} m_{2}+\boldsymbol{A}_{2} m_{1}} Q_{\boldsymbol{A}+\left(3 m_{1}+1,3 n_{1}+1\right)+\left(3 m_{2}-1,3 n_{2}-1\right)} P_{\boldsymbol{A}} \\
&+\sum_{\boldsymbol{B}} q^{n_{2} m_{1}+\boldsymbol{B}_{2} m_{2}+\boldsymbol{B}_{2} m_{1}} Q_{\left(3 m_{1}+1,3 n_{1}+1\right)+\boldsymbol{B}+\left(3 m_{2}-1,3 n_{2}-1\right)} P_{\boldsymbol{B}} \\
&= q^{n_{1} m_{2}}\left(\sum_{\boldsymbol{A}^{\prime}} q^{\left(n_{1}+n_{2}\right) \boldsymbol{A}_{1}^{\prime}} Q_{\boldsymbol{A}^{\prime}+\left(3 m_{1}+3 m_{2}, 3 n_{1}+3 n_{2}\right)} P_{\boldsymbol{A}^{\prime}}+\frac{1}{2} \delta_{\left(m_{1}, n_{1}\right),\left(-m_{2}, n_{2}\right)}\right) \\
& \quad-q^{n_{2} m_{1}}\left(-\sum_{\boldsymbol{A}} q^{\boldsymbol{A}_{2}\left(m_{2}+m_{1}\right)} Q_{\boldsymbol{A}+\left(3 m_{1}+3 m_{2}, 3 n_{1}+3 n_{2}\right)} P_{\boldsymbol{A}}\right. \\
& \quad\left.-\sum_{\boldsymbol{B}} q^{\boldsymbol{B}_{2}\left(m_{2}+m_{1}\right)} Q_{\boldsymbol{B}+\left(3 m_{1}+3 m_{2}, 3 n_{1}+3 n_{2}\right)} P_{\boldsymbol{B}}-\frac{1}{2} \delta_{\left(m_{1}, n_{1}\right),\left(-m_{2}, n_{2}\right)}\right) \\
&= q^{n_{1} m_{2}} e^{(\mu)}{ }_{11}\left(m_{1}+m_{2}, n_{1}+n_{2}\right)-q^{n_{2} m_{1}} e^{(\mu)}{ }_{22}\left(m_{1}+m_{2}, n_{1}+n_{2}\right) .
\end{aligned}
$$

The following six brackets can be checked easily.

$$
\begin{aligned}
& {\left[e^{(\mu)}{ }_{12}\left(m_{1}, n_{1}\right), e^{(\mu)}{ }_{22}\left(m_{2}, n_{2}\right)\right]=q^{n_{1} m_{2}} e^{(\mu)}{ }_{12}\left(m_{1}+m_{2}, n_{1}+n_{2}\right),} \\
& {\left[e^{(\mu)}{ }_{12}\left(m_{1}, n_{1}\right), e^{(\mu)}{ }_{23}\left(m_{2}, n_{2}\right)\right]=q^{n_{1} m_{2}} e^{(\mu)}{ }_{13}\left(m_{1}+m_{2}, n_{1}+n_{2}\right),} \\
& {\left[e^{(\mu)}{ }_{12}\left(m_{1}, n_{1}\right), e^{(\mu)}{ }_{31}\left(m_{2}, n_{2}\right)\right]=-q^{m_{1} n_{2}} e^{(\mu)}{ }_{32}\left(m_{1}+m_{2}, n_{1}+n_{2}\right),} \\
& {\left[e^{(\mu)}{ }_{12}\left(m_{1}, n_{1}\right), e^{(\mu)}{ }_{32}\left(m_{2}, n_{2}\right)\right]=0,} \\
& {\left[e^{(\mu)}{ }_{12}\left(m_{1}, n_{1}\right), e^{(\mu)}{ }_{33}\left(m_{2}, n_{2}\right)\right]=0,} \\
& {\left[e^{(\mu)}{ }_{13}\left(m_{1}, n_{1}\right), e^{(\mu)}{ }_{13}\left(m_{2}, n_{2}\right)\right]=0 .}
\end{aligned}
$$

Next,

$$
\begin{aligned}
& {\left[e^{(\mu)}{ }_{13}\left(m_{1}, n_{1}\right), e^{(\mu)}{ }_{21}\left(m_{2}, n_{2}\right)\right]} \\
& =\left[\sum_{\boldsymbol{B}} q^{\boldsymbol{B}_{1} n_{1}} Q_{\boldsymbol{B}+\left(3 m_{1}+2,3 n_{1}+2\right)} P_{\boldsymbol{B}},-q^{-m_{2} n_{2}} \mu P_{-\left(3 m_{2}-1,3 n_{2}-1\right)}\right. \\
& \quad-\sum_{\boldsymbol{A}, \boldsymbol{A}^{\prime}} q^{n_{2} \boldsymbol{A}_{1}^{\prime}+\boldsymbol{A}_{2} m_{2}+\boldsymbol{A}_{2} \boldsymbol{A}_{1}^{\prime}} Q_{\boldsymbol{A}+\boldsymbol{A}^{\prime}+\left(3 m_{2}-1,3 n_{2}-1\right)} P_{\boldsymbol{A}} P_{\boldsymbol{A}^{\prime}} \\
& \left.\quad-\sum_{\boldsymbol{A}, \boldsymbol{B}} q^{n_{2} \boldsymbol{A}_{1}+\boldsymbol{B}_{2} m_{2}+\boldsymbol{B}_{2} \boldsymbol{A}_{1}} Q_{\boldsymbol{A}+\boldsymbol{B}+\left(3 m_{2}-1,3 n_{2}-1\right)} P_{\boldsymbol{A}} P_{\boldsymbol{B}}\right] \\
& =-\sum_{\boldsymbol{A}, \boldsymbol{B}} q^{\left(\boldsymbol{A}_{1}+\boldsymbol{B}_{1}+m_{2}\right) n_{1}+n_{2}+\boldsymbol{A}_{1}+\boldsymbol{B}_{2} m_{2}+\boldsymbol{B}_{2} \boldsymbol{A}_{1}} Q_{\left(3 m_{1}+2,3 n_{1}+2\right)+\boldsymbol{A}+\boldsymbol{B}+\left(3 m_{2}-1,3 n_{2}-1\right)} P_{\boldsymbol{A}} P_{\boldsymbol{B}} \\
& +\sum_{\boldsymbol{A}, \boldsymbol{B}} q^{n_{2}\left(m_{1}+\boldsymbol{B}_{1}\right)+\boldsymbol{A}_{2} m_{2}+\boldsymbol{A}_{2}\left(m_{1}+\boldsymbol{B}_{1}\right)+\boldsymbol{B}_{1} n_{1}} Q_{\boldsymbol{A}+\left(3 m_{1}+2,3 n_{1}+2\right)+\boldsymbol{B}+\left(3 m_{2}-1,3 n_{2}-1\right)} P_{\boldsymbol{A}} P_{\boldsymbol{B}} \\
& \quad+q^{-m_{2} n_{2}+\left(-m_{1}-m_{2}\right) n_{1}} \mu P_{\left(-3 m_{1}-3 m_{2}-1,-3 n_{1}-3 n_{2}-1\right)}
\end{aligned}
$$


UNITARY REPRESENTATIONS OF THE EXTENDED AFFINE LIE ALGEBRA $\widetilde{\mathfrak{g l}_{3}\left(\mathbb{C}_{q}\right)} \quad 487$

$$
\begin{aligned}
& +\sum_{\boldsymbol{A}^{\prime}, \boldsymbol{B}} q^{n_{2} \boldsymbol{A}_{1}^{\prime}+\left(n_{1}+\boldsymbol{B}_{2}\right) m_{2}+\left(n_{1}+\boldsymbol{B}_{2}\right) \boldsymbol{A}_{1}^{\prime}+\boldsymbol{B}_{1} n_{1}} Q_{\boldsymbol{A}^{\prime}+\left(3 m_{1}+2,3 n_{1}+2\right)+\boldsymbol{B}+\left(3 m_{2}-1,3 n_{2}-1\right)} P_{\boldsymbol{A}^{\prime}} P_{\boldsymbol{B}} \\
+ & \sum_{\boldsymbol{B}, \boldsymbol{B}^{\prime}} q^{n_{2}\left(\boldsymbol{B}_{1}^{\prime}+m_{1}\right)+\boldsymbol{B}_{2} m_{2}+\boldsymbol{B}_{2}\left(\boldsymbol{B}_{1}^{\prime}+m_{1}\right)+\boldsymbol{B}_{1}^{\prime} n_{1}} Q_{\boldsymbol{B}^{\prime}+\left(3 m_{1}+2,3 n_{1}+2\right)+\boldsymbol{B}+\left(3 m_{2}-1,3 n_{2}-1\right)} P_{\boldsymbol{B}^{\prime}} P_{\boldsymbol{B}}
\end{aligned}
$$

(using that the first and fourth terms cancel each other)

$$
\begin{aligned}
& =q^{n_{2} m_{1}}\left(q^{-\left(m_{1}+m_{2}\right)\left(n_{1}+n_{2}\right)} \mu P_{-\left(3 m_{1}+3 m_{2}-1,3 n_{1}+3 n_{2}-1\right)}\right. \\
& \quad+\sum_{\boldsymbol{A}, \boldsymbol{A}^{\prime}} q^{\left(n_{1}+n_{2}\right) \boldsymbol{A}_{1}^{\prime}+\boldsymbol{A}_{2}\left(m_{1}+m_{2}\right)+\boldsymbol{A}_{2} \boldsymbol{A}_{1}^{\prime}} Q_{\boldsymbol{A}+\boldsymbol{A}^{\prime}+\left(3 m_{1}+3 m_{2}-1,3 n_{1}+3 n_{2}-1\right)} P_{\boldsymbol{A}} P_{\boldsymbol{A}^{\prime}} \\
& \left.\quad-\sum_{\boldsymbol{A}, \boldsymbol{B}} q^{\left(n_{1}+n_{2}\right) \boldsymbol{A}_{1}+\boldsymbol{B}_{2}\left(m_{1}+m_{2}\right)+\boldsymbol{B}_{2} \boldsymbol{A}_{1}} Q_{\boldsymbol{A}+\boldsymbol{B}+\left(3 m_{1}+3 m_{2}-1,3 n_{1}+3 n_{2}-1\right)} P_{\boldsymbol{A}} P_{\boldsymbol{B}}\right) \\
& =-q^{n_{2} m_{1}} e^{(\mu)}{ }_{23}\left(m_{1}+m_{2}, n_{1}+n_{2}\right) .
\end{aligned}
$$

The following two brackets are easy.

Next,

$$
\begin{aligned}
& {\left[e^{(\mu)}{ }_{13}\left(m_{1}, n_{1}\right), e^{(\mu)}{ }_{22}\left(m_{2}, n_{2}\right)\right]=0,} \\
& {\left[e^{(\mu)}{ }_{13}\left(m_{1}, n_{1}\right), e^{(\mu)}{ }_{23}\left(m_{2}, n_{2}\right)\right]=0 .}
\end{aligned}
$$

$$
\begin{aligned}
- & {\left[e^{(\mu)}{ }_{13}\left(m_{2}, n_{2}\right), e^{(\mu)}{ }_{31}\left(m_{1}, n_{1}\right)\right]=\left[e^{(\mu)} 31\left(m_{1}, n_{1}\right), e^{(\mu)}{ }_{13}\left(m_{2}, n_{2}\right)\right] } \\
= & \sum_{\boldsymbol{A}, \boldsymbol{B}} q^{n_{1} \boldsymbol{A}_{1}+n_{2} \boldsymbol{B}_{1}}\left[Q_{\left(3 m_{1}-2,3 n_{1}-2\right)+\boldsymbol{A}} P_{\boldsymbol{A}}, Q_{\left(3 m_{2}+2,3 n_{2}+2\right)+\boldsymbol{B}} P_{\boldsymbol{B}}\right] \\
= & \sum_{\boldsymbol{B}} q^{n_{2} \boldsymbol{B}_{1}+n_{1}\left(m_{2}+\boldsymbol{B}_{1}\right)} Q_{\left(3 m_{1}+3 m_{2}, 3 n_{1}+3 n_{2}\right)+\boldsymbol{B}} P_{\boldsymbol{B}} \\
& -\sum_{\boldsymbol{A}} q^{n_{1} \boldsymbol{A}_{1}+n_{2}\left(m_{1}+\boldsymbol{A}_{1}\right)} Q_{\left(3 m_{1}+3 m_{2}, 3 n_{1}+3 n_{2}\right)+\boldsymbol{A}} P_{\boldsymbol{A}} \\
= & q^{n_{1} m_{2}}\left(\sum_{\boldsymbol{B}} q^{n_{2} \boldsymbol{B}_{1}+n_{1}\left(m_{2}+\boldsymbol{B}_{1}\right)} Q_{\left(3 m_{1}+3 m_{2}, 3 n_{1}+3 n_{2}\right)+\boldsymbol{B}} P_{\boldsymbol{B}}+\frac{1}{2} \mu \delta_{\left(m_{1}+m_{2}, n_{1}+n_{2}\right),(0,0)}\right) \\
& -q^{n_{2} m_{1}}\left(\sum_{\boldsymbol{A}} q^{n_{1} \boldsymbol{A}_{1}+n_{2}\left(m_{1}+\boldsymbol{A}_{1}\right)} Q_{\left(3 m_{1}+3 m_{2}, 3 n_{1}+3 n_{2}\right)+\boldsymbol{A}} P_{\boldsymbol{A}}+\frac{1}{2} \mu \delta_{\left(m_{1}+m_{2}, n_{1}+n_{2}\right),(0,0)}\right) \\
= & q^{n_{1} m_{2}} e^{(\mu)} 33\left(m_{1}+m_{2}, n_{1}+n_{2}\right)-q^{n_{2} m_{1}} e^{(\mu)}{ }_{11}\left(m_{1}+m_{2}, n_{1}+n_{2}\right) .
\end{aligned}
$$

The next two brackets are easy.

$$
\begin{gathered}
{\left[e^{(\mu)}{ }_{13}\left(m_{1}, n_{1}\right), e^{(\mu)}{ }_{32}\left(m_{2}, n_{2}\right)\right]=q^{n_{1} m_{2}} e^{(\mu)}{ }_{12}\left(m_{1}+m_{2}, n_{1}+n_{2}\right),} \\
{\left[e^{(\mu)}{ }_{13}\left(m_{1}, n_{1}\right), e^{(\mu)}{ }_{33}\left(m_{2}, n_{2}\right)\right]=q^{n_{1} m_{2}} e^{(\mu)}{ }_{13}\left(m_{1}+m_{2}, n_{1}+n_{2}\right) .} \\
{\left[e^{(\mu)}{ }_{21}\left(m_{1}, n_{1}\right), e^{(\mu)}{ }_{21}\left(m_{2}, n_{2}\right)\right]} \\
=\mu q^{-m_{1} n_{1}} \sum_{\boldsymbol{A}} q^{\left(-m_{1}-m_{2}-\boldsymbol{A}_{1}\right) n_{2}+\boldsymbol{A}_{2} m_{2}+\boldsymbol{A}_{2}\left(-m_{1}-m_{2}-\boldsymbol{A}_{1}\right)} P_{\boldsymbol{A}} P_{\left(-3 m_{1}-3 m_{2},-3 n_{1}-3 n_{2}\right)-\boldsymbol{A}} \\
-\mu q^{-m_{2} n_{2}} \sum_{\boldsymbol{A}^{\prime}} q^{n_{1} \boldsymbol{A}_{1}^{\prime}+\left(-n_{1}-n_{2}-\boldsymbol{A}_{2}^{\prime}\right) m_{1}+\left(-n_{1}-n_{2}-\boldsymbol{A}_{2}^{\prime}\right) \boldsymbol{A}_{1}^{\prime}} \boldsymbol{P}_{\boldsymbol{A}^{\prime}} P_{\left(-3 m_{1}-3 m_{2},-3 n_{1}-3 n_{2}\right)-\boldsymbol{A}^{\prime}}
\end{gathered}
$$




$$
\begin{aligned}
& +\left[\sum_{\boldsymbol{A}, \boldsymbol{A}^{\prime}} q^{n_{1} \boldsymbol{A}_{1}^{\prime}+\boldsymbol{A}_{2} m_{1}+\boldsymbol{A}_{2} \boldsymbol{A}_{1}^{\prime}} Q_{\boldsymbol{A}+\boldsymbol{A}^{\prime}+\left(3 m_{1}-1,3 n_{1}-1\right)} P_{\boldsymbol{A}} P_{\boldsymbol{A}^{\prime}},\right. \\
& \left.\sum_{\boldsymbol{A}, \boldsymbol{A}^{\prime}} q^{n_{2} \boldsymbol{A}_{1}^{\prime}+\boldsymbol{A}_{2} m_{2}+\boldsymbol{A}_{2} \boldsymbol{A}_{1}^{\prime}} Q_{\boldsymbol{A}+\boldsymbol{A}^{\prime}+\left(3 m_{2}-1,3 n_{2}-1\right)} P_{\boldsymbol{A}} P_{\boldsymbol{A}^{\prime}}\right] \\
& +\left[\sum_{\boldsymbol{A}, \boldsymbol{A}^{\prime}} q^{n_{1} \boldsymbol{A}_{1}^{\prime}+\boldsymbol{A}_{2} m_{1}+\boldsymbol{A}_{2} \boldsymbol{A}_{1}^{\prime}} Q_{\boldsymbol{A}+\boldsymbol{A}^{\prime}+\left(3 m_{1}-1,3 n_{1}-1\right)} P_{\boldsymbol{A}} P_{\boldsymbol{A}^{\prime}},\right. \\
& \left.\sum_{\boldsymbol{A}, \boldsymbol{B}} q^{n_{2} \boldsymbol{A}_{1}+\boldsymbol{B}_{2} m_{2}+\boldsymbol{B}_{2} \boldsymbol{A}_{1}} Q_{\boldsymbol{A}+\boldsymbol{B}+\left(3 m_{2}-1,3 n_{2}-1\right)} P_{\boldsymbol{A}} P_{\boldsymbol{B}}\right] \\
& +\sum_{\boldsymbol{A}, \boldsymbol{B}} q^{n_{1} \boldsymbol{A}_{1}+\boldsymbol{B}_{2} m_{1}+\boldsymbol{B}_{2} \boldsymbol{A}_{1}} Q_{\boldsymbol{A}+\boldsymbol{B}+\left(3 m_{1}-1,3 n_{1}-1\right)} P_{\boldsymbol{A}} P_{\boldsymbol{B}}, \\
& +\left[\sum_{\boldsymbol{A}, \boldsymbol{A}, \boldsymbol{A}} q^{n_{2} \boldsymbol{A}_{1}^{\prime}+\boldsymbol{A}_{2} m_{2}+\boldsymbol{A}_{2} \boldsymbol{A}_{1}^{\prime}} Q_{\boldsymbol{A}+\boldsymbol{A}^{\prime}+\left(3 m_{2}-1,3 n_{2}-1\right)} P_{\boldsymbol{A}} P_{\boldsymbol{A}^{\prime}}\right] \\
& \sum_{\boldsymbol{A}+\boldsymbol{B}+\left(3 m_{1}-1,3 n_{1}-1\right)} P_{\boldsymbol{A}} P_{\boldsymbol{B}}, \\
& \left.\sum_{\boldsymbol{A}, \boldsymbol{B}} q^{n_{2} \boldsymbol{A}_{1}+\boldsymbol{B}_{2} m_{2}+\boldsymbol{B}_{2} \boldsymbol{A}_{1}} Q_{\boldsymbol{A}+\boldsymbol{B}+\left(3 m_{2}-1,3 n_{2}-1\right)} P_{\boldsymbol{A}} P_{\boldsymbol{B}}\right]
\end{aligned}
$$

(the first and second terms cancel each other)

$$
\begin{aligned}
& =\sum_{\overline{\boldsymbol{A}}^{\prime} \overline{\boldsymbol{A}}^{\prime}} q^{n_{1}\left(\overline{\boldsymbol{A}}_{1}+\overline{\boldsymbol{A}}_{1}^{\prime}+m_{2}\right)+\boldsymbol{A}_{2} m_{1}+\boldsymbol{A}_{2}\left(\overline{\boldsymbol{A}}_{1}+\overline{\boldsymbol{A}}_{1}^{\prime}+m_{2}\right)+n_{2} \overline{\boldsymbol{A}}_{1}^{\prime}+\overline{\boldsymbol{A}}_{2} m_{2}+\overline{\boldsymbol{A}}_{2} \overline{\boldsymbol{A}}_{1}^{\prime}} \\
& \boldsymbol{A}, \overline{\boldsymbol{A}}, \overline{\boldsymbol{A}}^{\prime} \quad \times Q_{\boldsymbol{A}+\overline{\boldsymbol{A}}+\overline{\boldsymbol{A}}^{\prime}+\left(3 m_{1}-1,3 n_{1}-1\right)+\left(3 m_{2}-1,3 n_{2}-1\right)} P_{\boldsymbol{A}} P_{\overline{\boldsymbol{A}}} P_{\overline{\boldsymbol{A}}^{\prime}} \\
& +\sum_{\boldsymbol{A}^{\prime}, \overline{\boldsymbol{A}}, \overline{\boldsymbol{A}}^{\prime}} q^{n_{1} \boldsymbol{A}_{1}^{\prime}+\left(\overline{\boldsymbol{A}}_{2}+\overline{\boldsymbol{A}}_{2}^{\prime}+n_{2}\right) m_{1}+\left(\overline{\boldsymbol{A}}_{2}+\overline{\boldsymbol{A}}_{2}^{\prime}+n_{2}\right) \boldsymbol{A}_{1}^{\prime}+n_{2} \overline{\boldsymbol{A}}_{1}^{\prime}+\overline{\boldsymbol{A}}_{2} m_{2}+\overline{\boldsymbol{A}}_{2} \overline{\boldsymbol{A}}_{1}^{\prime}} \\
& \times Q_{\boldsymbol{A}^{\prime}+\overline{\boldsymbol{A}}+\overline{\boldsymbol{A}}^{\prime}+\left(3 m_{1}-1,3 n_{1}-1\right)+\left(3 m_{2}-1,3 n_{2}-1\right)} P_{\boldsymbol{A}^{\prime}} P_{\overline{\boldsymbol{A}}} P_{\overline{\boldsymbol{A}}}^{\prime} \\
& -\sum q^{n_{2}\left(\boldsymbol{A}_{1}+\boldsymbol{A}_{1}^{\prime}+m_{1}\right)+\overline{\boldsymbol{A}}_{2} m_{2}+\overline{\boldsymbol{A}}_{2}\left(\boldsymbol{A}_{1}+\boldsymbol{A}_{1}^{\prime}+m_{1}\right)+n_{1} \boldsymbol{A}_{1}^{\prime}+\boldsymbol{A}_{2} m_{1}+\boldsymbol{A}_{2} \boldsymbol{A}_{1}^{\prime}} \\
& \underset{\boldsymbol{A}, \boldsymbol{A}^{\prime}, \overline{\boldsymbol{A}}}{ } \times Q_{\boldsymbol{A}+\boldsymbol{A}^{\prime}+\overline{\boldsymbol{A}}+\left(3 m_{1}-1,3 n_{1}-1\right)+\left(3 m_{2}-1,3 n_{2}-1\right)} P_{\boldsymbol{A}} P_{\boldsymbol{A}^{\prime}} P_{\overline{\boldsymbol{A}}} \\
& -\sum q^{n_{2} \overline{\boldsymbol{A}}_{1}^{\prime}+\left(\boldsymbol{A}_{2}+\boldsymbol{A}_{2}^{\prime}+n_{1}\right) m_{2}+\left(\boldsymbol{A}_{2}+\boldsymbol{A}_{2}^{\prime}+n_{1}\right) \overline{\boldsymbol{A}}_{1}^{\prime}+n_{1} \boldsymbol{A}_{1}^{\prime}+\boldsymbol{A}_{2} m_{1}+\boldsymbol{A}_{2} \boldsymbol{A}_{1}^{\prime}} \\
& \boldsymbol{A}, \boldsymbol{A}^{\prime}, \overline{\boldsymbol{A}}^{\prime} \quad \times Q_{\boldsymbol{A}+\boldsymbol{A}^{\prime}+\overline{\boldsymbol{A}}^{\prime}+\left(3 m_{1}-1,3 n_{1}-1\right)+\left(3 m_{2}-1,3 n_{2}-1\right)} P_{\boldsymbol{A}} P_{\boldsymbol{A}^{\prime}} P_{\overline{\boldsymbol{A}}^{\prime}} \\
& \begin{aligned}
-\sum_{\boldsymbol{A}, \boldsymbol{A}^{\prime}, \boldsymbol{B}} q^{n_{2}\left(\boldsymbol{A}_{1}+\boldsymbol{A}_{1}^{\prime}+m_{1}\right)+\boldsymbol{B}_{2} m_{2}+\boldsymbol{B}_{2}\left(\boldsymbol{A}_{1}+\boldsymbol{A}_{1}^{\prime}+m_{1}\right)+n_{1} \boldsymbol{A}_{1}^{\prime}+\boldsymbol{A}_{2} m_{1} \boldsymbol{A}_{2} \boldsymbol{A}_{1}^{\prime}} \\
\times Q_{\boldsymbol{A}+\boldsymbol{A}^{\prime}+\boldsymbol{B}+\left(3 m_{1}-1,3 n_{1}-1\right)+\left(3 m_{2}-1,3 n_{2}\right.}
\end{aligned} \\
& \begin{aligned}
+\sum_{\boldsymbol{A}, \boldsymbol{A}^{\prime}, \boldsymbol{B}} q^{n_{1}\left(\boldsymbol{A}_{1}+\boldsymbol{A}_{1}^{\prime}+m_{2}\right)+\boldsymbol{B}_{2} m_{1}+\boldsymbol{B}_{2}\left(\boldsymbol{A}_{1}+\boldsymbol{A}_{1}^{\prime}+m_{2}\right)+n_{2} \boldsymbol{A}_{1}^{\prime}+\boldsymbol{A}_{2} m_{2}+\boldsymbol{A}_{2} \boldsymbol{A}_{1}^{\prime}} \\
\times Q_{\boldsymbol{A}+\boldsymbol{A}^{\prime}+\boldsymbol{B}+\left(3 m_{1}-1,3 n_{1}-1\right)+\left(3 m_{2}-1,3 n_{2}-1\right)} P_{\boldsymbol{A}} P_{\boldsymbol{A}^{\prime}} P_{\boldsymbol{B}}
\end{aligned} \\
& \begin{aligned}
+\sum_{\boldsymbol{A}, \overline{\boldsymbol{A}}, \boldsymbol{B}} q^{n_{1} \boldsymbol{A}_{1}+\left(\overline{\boldsymbol{A}}_{2}+\boldsymbol{B}_{2}+n_{2}\right) m_{1}+\left(\overline{\boldsymbol{A}}_{2}+\boldsymbol{B}_{2}+n_{2}\right) \boldsymbol{A}_{1}+n_{2} \overline{\boldsymbol{A}}_{1}+\boldsymbol{B}_{2} m_{2}+\boldsymbol{B}_{2} \overline{\boldsymbol{A}}_{1}} \\
\times Q_{\boldsymbol{A}+\overline{\boldsymbol{A}}+\boldsymbol{B}+\left(3 m_{1}-1,3 n_{1}-1\right)+\left(3 m_{2}-1,3 n\right.}
\end{aligned} \\
& \begin{aligned}
-\sum_{\boldsymbol{A}, \overline{\boldsymbol{A}}, \overline{\boldsymbol{B}}} q^{n_{2} \boldsymbol{A}_{1}+\left(\overline{\boldsymbol{A}}_{2}+\overline{\boldsymbol{B}}_{2}+n_{1}\right) m_{2}+\left(\overline{\boldsymbol{A}}_{2}+\overline{\boldsymbol{B}}_{2}+n_{1}\right) \boldsymbol{A}_{1}+n_{1} \overline{\boldsymbol{A}}_{1}+\overline{\boldsymbol{B}}_{2} m_{1}+\overline{\boldsymbol{B}}_{2} \overline{\boldsymbol{A}}_{1}} \\
\times Q_{\boldsymbol{A}+\overline{\boldsymbol{A}}+\overline{\boldsymbol{B}}+\left(3 m_{1}-1,3 n_{1}-1\right)+\left(3 m_{2}-1,3 n_{2}-1\right)} P_{\boldsymbol{A}} P_{\overline{\boldsymbol{A}}} P_{\overline{\boldsymbol{B}}}
\end{aligned}
\end{aligned}
$$$$
=0,
$$

where we used that the first term cancels the fourth, the second term cancels the third, the fifth term cancels the seventh, and the sixth term cancels the eighth. Next, 
UNITARY REPRESENTATIONS OF THE EXTENDED AFFINE LIE ALGEBRA $\widetilde{\mathfrak{g l}_{3}\left(\mathbb{C}_{q}\right)} \quad 489$

$$
\begin{aligned}
& -\left[e^{(\mu)}{ }_{21}\left(m_{2}, n_{2}\right), e^{(\mu)}{ }_{22}\left(m_{1}, n_{1}\right)\right]=\left[e^{(\mu)}{ }_{22}\left(m_{1}, n_{1}\right), e^{(\mu)}{ }_{21}\left(m_{2}, n_{2}\right)\right] \\
& =\mu \sum_{\boldsymbol{A}} q^{\boldsymbol{A}_{2} m_{1}-m_{2} n_{2}}\left[Q_{\left(3 m_{1}, 3 n_{1}\right)+\boldsymbol{A}} P_{\boldsymbol{A}}, P_{-\left(3 m_{2}-1,3 n_{2}-1\right)}\right] \\
& +\sum_{\boldsymbol{A}, \boldsymbol{A}^{\prime}, \overline{\boldsymbol{A}}} q^{\boldsymbol{A}_{2} m_{1}+n_{2} \boldsymbol{A}_{1}^{\prime}+\overline{\boldsymbol{A}}_{2} m_{2}+\overline{\boldsymbol{A}}_{2} \boldsymbol{A}_{1}^{\prime}}\left[Q_{\left(3 m_{1}, 3 n_{1}\right)+\boldsymbol{A}} P_{\boldsymbol{A}}, Q_{\overline{\boldsymbol{A}}+\boldsymbol{A}^{\prime}+\left(3 m_{2}-1,3 n_{2}-1\right)} P_{\boldsymbol{A}^{\prime}} P_{\overline{\boldsymbol{A}}}\right] \\
& +\sum_{\boldsymbol{A}, \overline{\boldsymbol{A}}, \boldsymbol{B}} q^{\boldsymbol{A}_{2} m_{1}+n_{2} \overline{\boldsymbol{A}}_{1}+\boldsymbol{B}_{2} m_{2}+\boldsymbol{B}_{2} \overline{\boldsymbol{A}}_{1}}\left[Q_{\left(3 m_{1}, 3 n_{1}\right)+\boldsymbol{A}} P_{\boldsymbol{A}}, Q_{\overline{\boldsymbol{A}}+\boldsymbol{B}+\left(3 m_{2}-1,3 n_{2}-1\right)} P_{\boldsymbol{A}} P_{\boldsymbol{B}}\right] \\
& +\sum_{\boldsymbol{A}, \boldsymbol{B}, \overline{\boldsymbol{B}}} q^{\boldsymbol{B}_{2} m_{1}+n_{2} \boldsymbol{A}_{1}+\overline{\boldsymbol{B}}_{2} m_{2}+\overline{\boldsymbol{B}}_{2} \boldsymbol{A}_{1}}\left[Q_{\left(3 m_{1}, 3 n_{1}\right)+\boldsymbol{B}} P_{\boldsymbol{B}}, Q_{\boldsymbol{A}+\overline{\boldsymbol{B}}+\left(3 m_{2}-1,3 n_{2}-1\right)} P_{\boldsymbol{A}} P_{\overline{\boldsymbol{B}}}\right] \\
& =-\mu q^{-m_{2} n_{2}+\left(-n_{1}-n_{2}\right) m_{1}} P_{-3 m_{1}-3 m_{2}+1,-3 n_{1}-3 n_{2}+1} \\
& +\sum_{\boldsymbol{A}, \boldsymbol{A}^{\prime}} q^{\left(\boldsymbol{A}_{2}+\boldsymbol{A}_{2}^{\prime}+n_{2}\right) m_{1}+n_{2} \boldsymbol{A}_{1}^{\prime}+\boldsymbol{A}_{2} m_{2}+\boldsymbol{A}_{2} \boldsymbol{A}_{1}^{\prime}} Q_{\left(3 m_{1}, 3 n_{1}\right)+\boldsymbol{A}+\boldsymbol{A}^{\prime}+\left(3 m_{2}-1,3 n_{2}-1\right)} P_{\boldsymbol{A}} P_{\boldsymbol{A}^{\prime}} \\
& -\sum_{\boldsymbol{A}, \overline{\boldsymbol{A}}} q^{n_{2}\left(m_{1}+\overline{\boldsymbol{A}}_{1}\right)+\boldsymbol{A}_{2} m_{2}+\boldsymbol{A}_{2}\left(m_{1}+\overline{\boldsymbol{A}}_{1}\right)+\overline{\boldsymbol{A}}_{2} m_{1}} Q_{\boldsymbol{A}+\overline{\boldsymbol{A}}+\left(3 m_{1}, 3 n_{1}\right)+\left(3 m_{2}-1,3 n_{2}-1\right)} P_{\boldsymbol{A}} P_{\overline{\boldsymbol{A}}} \\
& -\sum_{\boldsymbol{A}^{\prime}, \overline{\boldsymbol{A}}} q^{n_{2} \boldsymbol{A}_{1}^{\prime}+\left(\overline{\boldsymbol{A}}_{2}+n_{1}\right) m_{2}+\left(\overline{\boldsymbol{A}}_{2}+n_{1}\right) \boldsymbol{A}_{1}^{\prime}+\overline{\boldsymbol{A}}_{2} m_{1}} Q_{\boldsymbol{A}^{\prime}+\overline{\boldsymbol{A}}+\left(3 m_{1}, 3 n_{1}\right)+\left(3 m_{2}-1,3 n_{2}-1\right)} P_{\boldsymbol{A}^{\prime}} P_{\overline{\boldsymbol{A}}} \\
& -\sum_{\boldsymbol{A}, \boldsymbol{B}} q^{n_{2}\left(\boldsymbol{A}_{1}+m_{1}\right)+\boldsymbol{B}_{2} m_{2}+\boldsymbol{B}_{2}\left(\boldsymbol{A}_{1}+m_{1}\right)+\boldsymbol{A}_{2} m_{1}} Q_{\boldsymbol{A}+\boldsymbol{B}+\left(3 m_{1}, 3 n_{1}\right)+\left(3 m_{2}-1,3 n_{2}-1\right)} P_{\boldsymbol{A}} P_{\boldsymbol{B}} \\
& +\sum_{\boldsymbol{A}, \boldsymbol{B}} q^{\left(\boldsymbol{A}_{2}+\boldsymbol{B}_{2}+n_{2}\right) m_{1}+n_{2} \boldsymbol{A}_{1}+\boldsymbol{B}_{2} m_{2}+\boldsymbol{B}_{2} \boldsymbol{A}_{1}} Q_{\boldsymbol{A}+\boldsymbol{B}+\left(3 m_{1}, 3 n_{1}\right)+\left(3 m_{2}-1,3 n_{2}-1\right)} P_{\boldsymbol{A}} P_{\boldsymbol{B}} \\
& -\sum_{\boldsymbol{A}, \boldsymbol{B}} q^{n_{2} \boldsymbol{A}_{1}+\left(n_{1}+\boldsymbol{B}_{2}\right) m_{2}+\left(n_{1}+\boldsymbol{B}_{2}\right) \boldsymbol{A}_{1}+\boldsymbol{B}_{2} m_{1}} Q_{\boldsymbol{A}+\boldsymbol{B}+\left(3 m_{1}, 3 n_{1}\right)+\left(3 m_{2}-1,3 n_{2}-1\right)} P_{\boldsymbol{A}} P_{\boldsymbol{B}}
\end{aligned}
$$

(using that the second and third terms cancel, as do the fifth and sixth)

$$
\begin{aligned}
= & q^{n_{1} m_{2}}\left(-\mu q^{-\left(n_{1}+n_{2}\right)\left(m_{1}+m_{2}\right)} P_{-\left(3\left(m_{1}+m_{2}\right)-1,3\left(n_{1}+n_{2}\right)-1\right)}\right. \\
& -\sum_{\overline{\boldsymbol{A}}, \boldsymbol{A}^{\prime}} q^{\left(n_{1}+n_{2}\right) \boldsymbol{A}_{1}^{\prime}+\left(m_{1}+m_{2}\right) \overline{\boldsymbol{A}}_{2}+\overline{\boldsymbol{A}}_{2} \boldsymbol{A}_{1}^{\prime}} Q_{\left(3 m_{1}+3 m_{2}-1,3 n_{1}+3 n_{2}-1\right)+\boldsymbol{A}^{\prime}+\overline{\boldsymbol{A}}} P_{\boldsymbol{A}^{\prime}} P_{\overline{\boldsymbol{A}}} \\
& \left.-\sum_{\boldsymbol{A}, \boldsymbol{B}} q^{\left(n_{1}+n_{2}\right) \boldsymbol{A}_{1}+\left(m_{1}+m_{2}\right) \boldsymbol{B}_{2}+\boldsymbol{B}_{2} \boldsymbol{A}_{1}}\right) Q_{\left(3 m_{1}+3 m_{2}-1,3 n_{1}+3 n_{2}-1\right)+\boldsymbol{A}+\boldsymbol{B}} P_{\boldsymbol{A}} P_{\boldsymbol{B}} \\
= & q^{n_{1} m_{2}} e^{(\mu)}{ }_{21}\left(m_{1}+m_{2}, n_{1}+n_{2}\right) .
\end{aligned}
$$

Further,

$$
\begin{aligned}
& {\left[e^{(\mu)}{ }_{21}\left(m_{1}, n_{1}\right), e^{(\mu)}{ }_{23}\left(m_{2}, n_{2}\right)\right]} \\
& =\mu q^{-m_{1} n_{1}} \sum_{\boldsymbol{A}} q^{n_{2}\left(-m_{1}-m_{2}-\boldsymbol{A}_{1}\right)+\boldsymbol{A}_{2} m_{2}+\boldsymbol{A}_{2}\left(-m_{1}-m_{2}-\boldsymbol{A}_{1}\right)} P_{\boldsymbol{A}} P_{\left(-3 m_{1}-3 m_{2},-3 n_{1}-3 n_{2}\right)-\boldsymbol{A}} \\
& +\left[\sum_{\boldsymbol{A}, \boldsymbol{A}^{\prime}} q^{n_{1} \boldsymbol{A}_{1}^{\prime}+\boldsymbol{A}_{2} m_{1}+\boldsymbol{A}_{2} \boldsymbol{A}_{1}^{\prime}} Q_{\boldsymbol{A}+\boldsymbol{A}^{\prime}+\left(3 m_{1}-1,3 n_{1}-1\right)} P_{\boldsymbol{A}} P_{\boldsymbol{A}^{\prime}},\right. \\
& \left.\sum_{\boldsymbol{A}, \boldsymbol{B}} q^{n_{2} \boldsymbol{B}_{1}+\boldsymbol{A}_{2} m_{2}+\boldsymbol{A}_{2} \boldsymbol{B}_{1}} Q_{\left(3 m_{2}+1,3 n_{2}+1\right)+\boldsymbol{A}+\boldsymbol{B}} P_{\boldsymbol{A}} P_{\boldsymbol{B}}\right]
\end{aligned}
$$




$$
\begin{gathered}
-\mu q^{-m_{2} n_{2}} \sum_{\boldsymbol{A}} q^{n_{1} \boldsymbol{A}_{1}+\left(-n_{1}-n_{2}-\boldsymbol{A}_{2}\right) m_{1}+\left(-n_{1}-n_{2}-\boldsymbol{A}_{2}\right) \boldsymbol{A}_{1}} P_{\boldsymbol{A}} P_{\left(-3 m_{1}-3 m_{2},-3 n_{1}-3 n_{2}\right)-\boldsymbol{A}} \\
+\left[\sum_{\boldsymbol{A}, \boldsymbol{B}} q^{n_{1} \boldsymbol{A}_{1}+\boldsymbol{B}_{2} m_{1}+\boldsymbol{B}_{2} \boldsymbol{A}_{1}} Q_{\boldsymbol{A}+\boldsymbol{B}+\left(3 m_{1}-1,3 n_{1}-1\right)} P_{\boldsymbol{A}} P_{\boldsymbol{B}},\right. \\
\left.\sum_{\boldsymbol{A}, \boldsymbol{B}} q^{n_{2} \boldsymbol{B}_{1}+\boldsymbol{A}_{2} m_{2}+\boldsymbol{A}_{2} \boldsymbol{B}_{1}} Q_{\left(3 m_{2}+1,3 n_{2}+1\right)} P_{\boldsymbol{A}} P_{\boldsymbol{B}}\right] \\
+\left[\sum_{\boldsymbol{A}, \boldsymbol{B}} q^{n_{1} \boldsymbol{A}_{1}+\boldsymbol{B}_{2} m_{1}+\boldsymbol{B}_{2} \boldsymbol{A}_{1}} Q_{\boldsymbol{A}+\boldsymbol{B}+\left(3 m_{1}-1,3 n_{1}-1\right)} P_{\boldsymbol{A}} P_{\boldsymbol{B}},\right. \\
\sum_{\boldsymbol{B}, \boldsymbol{B}^{\prime}} q^{n_{2} \boldsymbol{B}_{1}^{\prime}+\boldsymbol{B}_{2} m_{2}+\boldsymbol{B}_{2} \boldsymbol{B}_{1}^{\prime}} Q_{\left.\left(3 m_{2}+1,3 n_{2}+1\right)+\boldsymbol{B}+\boldsymbol{B}^{\prime} P_{\boldsymbol{B}} P_{\boldsymbol{B}^{\prime}}\right]}
\end{gathered}
$$

(the first and third term cancel)

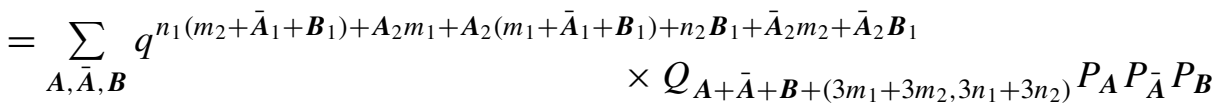

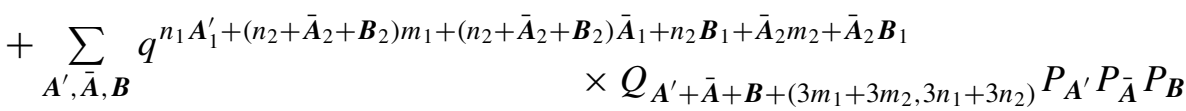

$$
\begin{aligned}
& \begin{aligned}
-\sum_{\boldsymbol{A}, \boldsymbol{A}^{\prime}, \boldsymbol{B}} q^{n_{2} \boldsymbol{B}_{1}+\left(\boldsymbol{A}_{2}+\boldsymbol{A}_{2}^{\prime}+n_{1}\right) m_{2}+\left(\boldsymbol{A}_{2}+\boldsymbol{A}_{2}^{\prime}+n_{1}\right) \boldsymbol{B}_{1}+n_{1} \boldsymbol{A}_{1}^{\prime}+\boldsymbol{A}_{2} m_{1}+\boldsymbol{A}_{2} \boldsymbol{A}_{1}^{\prime}} \\
\times Q_{\boldsymbol{A}^{\prime}+\boldsymbol{A}+\boldsymbol{B}+\left(3 m_{1}+3 m_{2}, 3 n_{1}+3 n_{2}\right)} P_{\boldsymbol{A}^{\prime}} P_{\boldsymbol{A}} P_{\boldsymbol{B}}
\end{aligned} \\
& +\sum_{\boldsymbol{B}, \boldsymbol{B}^{\prime}} q^{n_{1}\left(m_{2}+\boldsymbol{A}_{1}+\boldsymbol{B}_{1}^{\prime}\right)+\boldsymbol{B}_{2} m_{1}+\boldsymbol{B}_{2}\left(m_{2}+\boldsymbol{A}_{1}+\boldsymbol{B}_{1}^{\prime}\right)+n_{2} \boldsymbol{B}_{1}^{\prime}+\boldsymbol{A}_{2} m_{2}+\boldsymbol{A}_{2} \boldsymbol{B}_{1}^{\prime}} \\
& \sum_{\boldsymbol{A}, \boldsymbol{B}, \boldsymbol{B}^{\prime}} \times Q_{\boldsymbol{A}+\boldsymbol{B}+\boldsymbol{B}^{\prime}+\left(3 m_{1}+3 m_{2}, 3 n_{1}+3 n_{2}\right)} P_{\boldsymbol{A}} P_{\boldsymbol{B}} P_{\boldsymbol{B}^{\prime}} \\
& \begin{aligned}
-\sum_{\boldsymbol{A}, \overline{\boldsymbol{A}}, \boldsymbol{B}} q^{n_{2}\left(\boldsymbol{A}_{1}+\boldsymbol{B}_{1}+m_{1}\right)+\overline{\boldsymbol{A}}_{2} m_{2}+\overline{\boldsymbol{A}}_{2}\left(\boldsymbol{A}_{1}+\boldsymbol{B}+m_{1}\right)+n_{1} \boldsymbol{A}_{1}+\boldsymbol{B}_{2} m_{1}+\boldsymbol{B}_{2} \boldsymbol{A}_{1}} \\
\times Q_{\boldsymbol{A}+\overline{\boldsymbol{A}}+\boldsymbol{B}+\left(3 m_{1}+3 m_{2}, 3 n_{1}+3 n_{2}\right)} P_{\boldsymbol{A}} P_{\overline{\boldsymbol{A}}} P_{\boldsymbol{B}}
\end{aligned} \\
& \begin{aligned}
+\sum_{\boldsymbol{A}, \boldsymbol{B}, \boldsymbol{B}^{\prime}} q^{n_{1} \boldsymbol{A}_{1}+\left(n_{2}+\boldsymbol{B}_{2}+\boldsymbol{B}_{2}^{\prime}\right) m_{1}+\left(n_{2}+\boldsymbol{B}_{2}+\boldsymbol{B}_{2}^{\prime}\right) \boldsymbol{A}_{1}+n_{2} \boldsymbol{B}_{1}^{\prime}+\boldsymbol{B}_{2} m_{2}+\boldsymbol{B}_{2} \boldsymbol{B}_{1}^{\prime}} & \times Q_{\boldsymbol{A}+\boldsymbol{B}+\boldsymbol{B}^{\prime}+\left(3 m_{1}+3 m_{2}, 3 n_{1}+3 n_{2}\right)} P_{\boldsymbol{A}} P_{\boldsymbol{B}} P_{\boldsymbol{B}^{\prime}}
\end{aligned} \\
& \begin{aligned}
-\sum_{\boldsymbol{A}, \boldsymbol{B}, \overline{\boldsymbol{B}}} q^{n_{2}\left(\boldsymbol{A}_{1}+\overline{\boldsymbol{B}}_{1}+m_{1}\right)+\boldsymbol{B}_{2} m_{2}+\boldsymbol{B}_{2}\left(\boldsymbol{A}_{1}+\overline{\boldsymbol{B}}_{1}+m_{1}\right)+n_{1} \boldsymbol{A}_{1}+\overline{\boldsymbol{B}}_{2} m_{1}+\overline{\boldsymbol{B}}_{2} \boldsymbol{A}_{1}} \\
\times Q_{\boldsymbol{A}+\boldsymbol{B}+\overline{\boldsymbol{B}}+\left(3 m_{1}+3 m_{2}, 3 n_{1}+3 n_{2}\right)} P_{\boldsymbol{A}} P_{\boldsymbol{B}} P_{\overline{\boldsymbol{B}}}
\end{aligned} \\
& \begin{aligned}
-\sum_{\boldsymbol{A}, \boldsymbol{B}^{\prime}, \overline{\boldsymbol{B}}} q^{n_{2} \boldsymbol{B}_{1}^{\prime}+\left(\boldsymbol{A}_{2}+\overline{\boldsymbol{B}}_{2}+n_{1}\right) m_{2}+\left(\boldsymbol{A}_{2}+\overline{\boldsymbol{B}}_{2}+n_{1}\right) \boldsymbol{B}_{1}^{\prime}+n_{1} \boldsymbol{A}_{1}+\overline{\boldsymbol{B}}_{2} m_{1}+\overline{\boldsymbol{B}}_{2} \boldsymbol{A}_{1}} \\
\times Q_{\boldsymbol{A}+\boldsymbol{B}^{\prime}+\overline{\boldsymbol{B}}+\left(3 m_{1}+3 m_{2}, 3 n_{1}+3 n_{2}\right)} P_{\boldsymbol{A}} P_{\boldsymbol{B}^{\prime}} P_{\overline{\boldsymbol{B}}}
\end{aligned} \\
& =0 \text {, }
\end{aligned}
$$

where the first term and the third, the second and the fifth, the fourth and the eighth, and the sixth and the seventh cancel.

The following three brackets are easy.

$$
\begin{aligned}
& {\left[e^{(\mu)}{ }_{21}\left(m_{1}, n_{1}\right), e^{(\mu)}{ }_{31}\left(m_{2}, n_{2}\right)\right]=0,} \\
& {\left[e^{(\mu)}{ }_{21}\left(m_{1}, n_{1}\right), e^{(\mu)}{ }_{32}\left(m_{2}, n_{2}\right)\right]=-q^{n_{2} m_{1}} e^{(\mu)}{ }_{31}\left(m_{1}+m_{2}, n_{1}+n_{2}\right),} \\
& {\left[e^{(\mu)}{ }_{21}\left(m_{1}, n_{1}\right), e^{(\mu)}{ }_{33}\left(m_{2}, n_{2}\right)\right]=0 .}
\end{aligned}
$$




\section{Next,}

$$
\begin{aligned}
& {\left[e^{(\mu)}{ }_{22}\left(m_{1}, n_{1}\right), e^{(\mu)}{ }_{22}\left(m_{2}, n_{2}\right)\right]} \\
& =\sum_{\boldsymbol{A}, \boldsymbol{A}^{\prime}} q^{\boldsymbol{A}_{2} m_{1}+\boldsymbol{A}_{2}^{\prime} m_{2}}\left[Q_{\left(3 m_{1}, 3 n_{1}\right)+\boldsymbol{A}} P_{\boldsymbol{A}}, Q_{\left(3 m_{2}, 3 n_{2}\right)+\boldsymbol{A}^{\prime}} P_{\boldsymbol{A}^{\prime}}\right] \\
& +\sum_{\boldsymbol{B}, \boldsymbol{B}^{\prime}} q^{\boldsymbol{B}_{2} m_{1}+\boldsymbol{B}_{2}^{\prime} m_{2}}\left[Q_{\left(3 m_{1}, 3 n_{1}\right)+\boldsymbol{B}} P_{\boldsymbol{B}}, Q_{\left(3 m_{2}, 3 n_{2}\right)+\boldsymbol{B}^{\prime}} P_{\boldsymbol{B}^{\prime}}\right] \\
& =\sum_{\boldsymbol{A}^{\prime}} q^{\left(n_{2}+\boldsymbol{A}_{2}^{\prime}\right) m_{1}+\boldsymbol{A}_{2}^{\prime} m_{2}} Q_{\left(3 m_{1}, 3 n_{1}\right)+\left(3 m_{2}, 3 n_{2}\right)+\boldsymbol{A}^{\prime}} \boldsymbol{P}_{\boldsymbol{A}^{\prime}} \\
& -\sum_{\boldsymbol{A}} q^{\boldsymbol{A}_{2} m_{1}+\left(n_{1}+\boldsymbol{A}_{2}\right) m_{2}} Q_{\left(3 m_{1}, 3 n_{1}\right)+\left(3 m_{2}, 3 n_{2}\right)+\boldsymbol{A}} P_{\boldsymbol{A}} \\
& +\sum_{\boldsymbol{B}^{\prime}} q^{\left(n_{2}+\boldsymbol{B}_{2}^{\prime}\right) m_{1}+\boldsymbol{B}_{2}^{\prime} m_{2}} Q_{\left(3 m_{1}, 3 n_{1}\right)+\left(3 m_{2}, 3 n_{2}\right)+\boldsymbol{B}^{\prime}} P_{\boldsymbol{B}^{\prime}} \\
& -\sum_{\boldsymbol{B}} q^{\boldsymbol{B}_{2} m_{1}+\left(n_{1}+\boldsymbol{B}_{2}\right) m_{2}} Q_{\left(3 m_{1}, 3 n_{1}\right)+\left(3 m_{2}, 3 n_{2}\right)+\boldsymbol{B}} P_{\boldsymbol{B}} \\
& =q^{n_{1} m_{2}}\left(-\sum_{\boldsymbol{A}} q^{\boldsymbol{A}_{2}\left(m_{1}+m_{2}\right)} Q_{\left(3 m_{1}, 3 n_{1}\right)+\left(3 m_{2}, 3 n_{2}\right)+\boldsymbol{A}} P_{\boldsymbol{A}}\right. \\
& \left.-\sum_{\boldsymbol{B}} q^{\boldsymbol{B}_{2}\left(m_{1}+m_{2}\right)} Q_{\left(3 m_{1}, 3 n_{1}\right)+\left(3 m_{2}, 3 n_{2}\right)+\boldsymbol{B}} P_{\boldsymbol{B}}-\frac{1}{2} \mu \delta_{\left(m_{1}+m_{2}, n_{1}+n_{2}\right),(0,0)}\right) \\
& -q^{n_{2} m_{1}}\left(-\sum_{\boldsymbol{A}} q^{\boldsymbol{A}_{2}\left(m_{1}+m_{2}\right)} Q_{\left(3 m_{1}, 3 n_{1}\right)+\left(3 m_{2}, 3 n_{2}\right)+\boldsymbol{A}} P_{\boldsymbol{A}}\right. \\
& \left.-\sum_{\boldsymbol{B}} q^{\boldsymbol{B}_{2}\left(m_{1}+m_{2}\right)} Q_{\left(3 m_{1}, 3 n_{1}\right)+\left(3 m_{2}, 3 n_{2}\right)+\boldsymbol{B}} P_{\boldsymbol{B}}-\frac{1}{2} \mu \delta_{\left(m_{1}+m_{2}, n_{1}+n_{2}\right),(0,0)}\right) \\
& =q^{n_{1} m_{2}} e^{(\mu)}{ }_{22}\left(m_{1}+m_{2}, n_{1}+n_{2}\right)-q^{n_{2} m_{1}} e^{(\mu)}{ }_{22}\left(m_{1}+m_{2}, n_{1}+n_{2}\right) \text {. }
\end{aligned}
$$

$$
\begin{aligned}
& {\left[e^{(\mu)}{ }_{22}\left(m_{1}, n_{1}\right), e^{(\mu)}{ }_{23}\left(m_{2}, n_{2}\right)\right]} \\
& =\left[-\sum_{\boldsymbol{A}} q^{\boldsymbol{A}_{2} m_{1}} Q_{\left(3 m_{1}, 3 n_{1}\right)+\boldsymbol{A}} P_{\boldsymbol{A}}-\sum_{\boldsymbol{B}} q^{\boldsymbol{B}_{2} m_{1}} Q_{\left(3 m_{1}, 3 n_{1}\right)+\boldsymbol{B}} P_{\boldsymbol{B}},\right. \\
& -q^{-m_{2} n_{2}} \mu P_{-\left(3 m_{2}+1,3 n_{2}+1\right)}-\sum_{\boldsymbol{A}, \boldsymbol{B}} q^{n_{2} \boldsymbol{B}_{1}+\boldsymbol{A}_{2} m_{2}+\boldsymbol{A}_{2} \boldsymbol{B}_{1}} Q_{\boldsymbol{A}+\boldsymbol{B}+\left(3 m_{2}+1,3 n_{2}+1\right)} P_{\boldsymbol{A}} P_{\boldsymbol{B}} \\
& \left.-\sum_{\boldsymbol{B}, \boldsymbol{B}^{\prime}} q^{n_{2} \boldsymbol{B}_{1}^{\prime}+\boldsymbol{B}_{2} m_{2}+\boldsymbol{B}_{2} \boldsymbol{B}_{1}^{\prime}} Q_{\boldsymbol{B}^{\prime}+\boldsymbol{B}+\left(3 m_{2}+1,3 n_{2}+1\right)} P_{\boldsymbol{B}} P_{\boldsymbol{B}^{\prime}}\right] \\
& =\sum_{\boldsymbol{A}, \boldsymbol{B}} q^{\left(n_{2}+\boldsymbol{A}_{2}+\boldsymbol{B}_{2}\right) m_{1}+n_{2} \boldsymbol{B}_{1}+\boldsymbol{A}_{2} m_{2}+\boldsymbol{A}_{2} \boldsymbol{B}_{1}} Q_{\left(3 m_{1}, 3 n_{1}\right)+\boldsymbol{A}+\boldsymbol{B}+\left(3 m_{2}+1,3 n_{2}+1\right)} P_{\boldsymbol{A}} P_{\boldsymbol{B}} \\
& -\sum_{\boldsymbol{A}, \boldsymbol{B}} q^{n_{2} \boldsymbol{B}_{1}+\left(n_{1}+\boldsymbol{A}_{2}\right) m_{2}+\left(\boldsymbol{A}_{2}+n_{1}\right) \boldsymbol{B}_{1}+\boldsymbol{A}_{2} m_{1}} Q_{\left(3 m_{1}, 3 n_{1}\right)+\boldsymbol{A}+\boldsymbol{B}+\left(3 m_{2}+1,3 n_{2}+1\right)} P_{\boldsymbol{A}} P_{\boldsymbol{B}} \\
& -q^{-m_{2} n_{2}+\left(-n_{2}-n_{1}\right) m_{1}} \mu P_{-\left(3 m_{2}+1,3 n_{2}+1\right)-\left(3 m_{1}, 3 n_{1}\right)} \\
& -\sum_{\boldsymbol{A}, \boldsymbol{B}} q^{n_{2}\left(m_{1}+\boldsymbol{B}_{1}\right)+\boldsymbol{A}_{2} m_{2}+\boldsymbol{A}_{2}\left(m_{1}+\boldsymbol{B}_{1}\right)+\boldsymbol{B}_{2} m_{1}} Q_{\left(3 m_{1}, 3 n_{1}\right)+\boldsymbol{A}+\boldsymbol{B}+\left(3 m_{2}+1,3 n_{2}+1\right)} P_{\boldsymbol{A}} P_{\boldsymbol{B}} \\
& +\sum_{\boldsymbol{B}, \boldsymbol{B}^{\prime}} q^{\left(n_{2}+\boldsymbol{B}_{2}+\boldsymbol{B}_{2}^{\prime}\right) m_{1}+n_{2} \boldsymbol{B}_{1}^{\prime}+\boldsymbol{B}_{2} m_{2}+\boldsymbol{B}_{2} \boldsymbol{B}_{1}^{\prime}} Q_{\left(3 m_{1}, 3 n_{1}\right)+\boldsymbol{B}+\boldsymbol{B}^{\prime}+\left(3 m_{2}+1,3 n_{2}+1\right)} P_{\boldsymbol{B}} P_{\boldsymbol{B}^{\prime}}
\end{aligned}
$$


$-\sum_{\boldsymbol{B}, \boldsymbol{B}^{\prime}} q^{n_{2}\left(\boldsymbol{B}_{1}^{\prime}+m_{1}\right)+\boldsymbol{B}_{2} m_{2}+\boldsymbol{B}_{2}\left(\boldsymbol{B}_{1}^{\prime}+m_{1}\right)+\boldsymbol{B}_{2}^{\prime} m_{1}} Q_{\left(3 m_{1}, 3 n_{1}\right)+\boldsymbol{B}+\boldsymbol{B}^{\prime}+\left(3 m_{2}+1,3 n_{2}+1\right)} P_{\boldsymbol{B}} P_{\boldsymbol{B}^{\prime}}$
$-\sum_{\boldsymbol{B}, \boldsymbol{B}^{\prime}} q^{n_{2} \boldsymbol{B}_{1}^{\prime}+\left(n_{1}+\boldsymbol{B}_{2}\right) m_{2}+\left(\boldsymbol{B}_{2}+n_{1}\right) \boldsymbol{B}_{1}^{\prime}+\boldsymbol{B}_{2} m_{1}} Q_{\left(3 m_{1}, 3 n_{1}\right)+\boldsymbol{B}+\boldsymbol{B}^{\prime}+\left(3 m_{2}+1,3 n_{2}+1\right)} P_{\boldsymbol{B}} P_{\boldsymbol{B}^{\prime}}$ (the first term cancels the fourth, while the fifth cancels the sixth)

$$
\begin{aligned}
= & q^{m_{2} n_{1}}\left(-q^{-\left(m_{1}+m_{2}\right)\left(n_{1}+n_{2}\right)} \mu P_{-\left(3 m_{1}+3 m_{2}+1,3 n_{1}+3 n_{2}+1\right)}\right. \\
& -\sum_{\boldsymbol{A}, \boldsymbol{B}} q^{\boldsymbol{A}_{2}\left(m_{1}+m_{2}\right)+\left(n_{1}+n_{2}\right) \boldsymbol{B}_{1}+\boldsymbol{A}_{2} \boldsymbol{B}_{1}} Q_{\boldsymbol{A}+\boldsymbol{B}+\left(3 m_{1}+3 m_{2}+1,3 n_{1}+3 n_{2}+1\right)} P_{\boldsymbol{A}} P_{\boldsymbol{B}} \\
& -\sum_{\boldsymbol{B}, \boldsymbol{B}^{\prime}} q^{\left.\boldsymbol{B}_{1}^{\prime}\left(n_{1}+n_{2}\right)+\boldsymbol{B}_{2}\left(m_{1}+m_{2}\right) \boldsymbol{B}_{2} \boldsymbol{B}_{1}^{\prime}\right)} Q_{\boldsymbol{B}+\boldsymbol{B}^{\prime}+\left(3 m_{1}+3 m_{2}+1,3 n_{1}+3 n_{2}+1\right)} P_{\boldsymbol{B}} P_{\boldsymbol{B}^{\prime}} \\
= & q^{m_{2} n_{1}} e^{(\mu)}{ }_{23}\left(m_{1}+m_{2}, n_{1}+n_{2}\right) .
\end{aligned}
$$

The next three brackets are easy.

$$
\begin{aligned}
& {\left[e^{(\mu)}{ }_{22}\left(m_{1}, n_{1}\right), e^{(\mu)}{ }_{31}\left(m_{2}, n_{2}\right)\right]=0,} \\
& {\left[e^{(\mu)}{ }_{22}\left(m_{1}, n_{1}\right), e^{(\mu)} 32\left(m_{2}, n_{2}\right)\right]=-q^{n_{2} m_{1}} e^{(\mu)} 32\left(m_{1}+m_{2}, n_{1}+n_{2}\right),} \\
& {\left[e^{(\mu)}{ }_{22}\left(m_{1}, n_{1}\right), e^{(\mu)}{ }_{33}\left(m_{2}, n_{2}\right)\right]=0 .}
\end{aligned}
$$

Next,

$$
\begin{aligned}
& {\left[e^{(\mu)}{ }_{23}\left(m_{1}, n_{1}\right), e^{(\mu)}{ }_{23}\left(m_{2}, n_{2}\right)\right]} \\
& =\left[-q^{-m_{1} n_{1}} \mu P_{-\left(3 m_{1}+1,3 n_{1}+1\right)}-\sum_{\boldsymbol{A}, \boldsymbol{B}} q^{n_{1} \boldsymbol{B}_{1}+\boldsymbol{A}_{2} m_{1}+\boldsymbol{A}_{2} \boldsymbol{B}_{1}} Q_{\boldsymbol{A}+\boldsymbol{B}+\left(3 m_{1}+1,3 n_{1}+1\right)} P_{\boldsymbol{A}} P_{\boldsymbol{B}}\right. \\
& -\sum_{\boldsymbol{B}, \boldsymbol{B}^{\prime}} q^{n_{1} \boldsymbol{B}_{1}^{\prime}+\boldsymbol{B}_{2} m_{1}+\boldsymbol{B}_{2} \boldsymbol{B}_{1}^{\prime}} Q_{\boldsymbol{B}+\boldsymbol{B}^{\prime}+\left(3 m_{1}+1,3 n_{1}+1\right)} P_{\boldsymbol{B}} P_{\boldsymbol{B}^{\prime}} \\
& -q^{-m_{2} n_{2}} \mu P_{-\left(3 m_{2}+1,3 n_{2}+1\right)}-\sum_{\boldsymbol{A}, \boldsymbol{B}} q^{n_{2} \boldsymbol{B}_{1}+\boldsymbol{A}_{2} m_{2}+\boldsymbol{A}_{2} \boldsymbol{B}_{1}} Q_{\boldsymbol{A}+\boldsymbol{B}+\left(3 m_{2}+1,3 n_{2}+1\right)} P_{\boldsymbol{A}} P_{\boldsymbol{B}} \\
& \left.-\sum_{\boldsymbol{B}, \boldsymbol{B}^{\prime}} q^{n_{2} \boldsymbol{B}_{1}^{\prime}+\boldsymbol{B}_{2} m_{2}+\boldsymbol{B}_{2} \boldsymbol{B}_{1}^{\prime}} Q_{\boldsymbol{B}+\boldsymbol{B}^{\prime}+\left(3 m_{2}+1,3 n_{2}+1\right)} P_{\boldsymbol{B}} P_{\boldsymbol{B}^{\prime}}\right] \\
& =\sum_{\boldsymbol{B}} \mu q^{-m_{1} n_{1}+n_{2}\left(-m_{1}-m_{2}-\boldsymbol{B}_{1}\right)+\boldsymbol{B}_{2} m_{2}+\boldsymbol{B}_{2}\left(-m_{1}-m_{2}-\boldsymbol{B}_{1}\right)} \\
& \times P_{\boldsymbol{B}} P_{-}\left(3 m_{1}+1,3 n_{1}+1\right)-\left(3 m_{2}+1,3 n_{2}+1\right)-\boldsymbol{B} \\
& \begin{aligned}
-\sum_{\boldsymbol{B}} \mu q^{-m_{2} n_{2}+n_{1} \boldsymbol{B}_{1}+\left(-n_{2}-n_{1}-\boldsymbol{B}_{2}\right) m_{1}+\left(-n_{2}-n_{1}-\boldsymbol{B}_{2}\right) \boldsymbol{B}_{1}} & \\
& \times P_{\boldsymbol{B}} P_{-\left(3 m_{1}+1,3 n_{1}+1\right)-\left(3 m_{2}+1,3 n_{2}+1\right)-\boldsymbol{B}}
\end{aligned} \\
& \begin{aligned}
+\sum_{\boldsymbol{B}, \overline{\boldsymbol{B}}, \boldsymbol{B}^{\prime}} q^{n_{1}\left(\boldsymbol{B}_{1}^{\prime}+\overline{\boldsymbol{B}}_{1}+m_{2}\right)+\boldsymbol{B}_{2} m_{1}+\boldsymbol{B}_{2}\left(\boldsymbol{B}_{1}^{\prime}+\overline{\boldsymbol{B}}_{1}+m_{2}\right)+n_{2} \boldsymbol{B}_{1}^{\prime}+\overline{\boldsymbol{B}}_{2} m_{2}+\overline{\boldsymbol{B}}_{2} \boldsymbol{B}_{1}^{\prime}} & \\
& \times Q_{\boldsymbol{B}+\boldsymbol{B}^{\prime}+\overline{\boldsymbol{B}}+\left(3 m_{1}+1,3 n_{1}+1\right)+\left(3 m_{2}+1,3 n_{2}+1\right)} P_{\boldsymbol{B}} P_{\boldsymbol{B}^{\prime}} P_{\overline{\boldsymbol{B}}}
\end{aligned} \\
& \begin{array}{r}
+\sum_{\boldsymbol{B}, \overline{\boldsymbol{B}}, \boldsymbol{B}^{\prime}} q^{n_{1} \boldsymbol{B}_{1}^{\prime}+\left(\boldsymbol{B}_{2}+\overline{\boldsymbol{B}}_{2}+n_{2}\right) m_{1}+\boldsymbol{B}_{1}^{\prime}\left(\boldsymbol{B}_{2}+\overline{\boldsymbol{B}}_{2}+n_{2}\right)+n_{2} \overline{\boldsymbol{B}}_{1}+\boldsymbol{B}_{2} m_{2}+\boldsymbol{B}_{2} \overline{\boldsymbol{B}}_{1}} \\
\times Q_{\boldsymbol{B}+\boldsymbol{B}^{\prime}+\overline{\boldsymbol{B}}+\left(3 m_{1}+1,3 n_{1}+1\right)+\left(3 m_{2}+1\right.}
\end{array} \\
& \times Q_{\boldsymbol{B}+\boldsymbol{B}^{\prime}+\overline{\boldsymbol{B}}+\left(3 m_{1}+1,3 n_{1}+1\right)+\left(3 m_{2}+1,3 n_{2}+1\right)} P_{\boldsymbol{B}} P_{\boldsymbol{B}^{\prime}} P_{\overline{\boldsymbol{B}}}
\end{aligned}
$$




$$
\begin{aligned}
&-\sum_{\boldsymbol{B}, \overline{\boldsymbol{B}}, \boldsymbol{B}^{\prime}} q^{n_{2}\left(\boldsymbol{B}_{1}^{\prime}+\overline{\boldsymbol{B}}_{1}+m_{1}\right)+\boldsymbol{B}_{2} m_{2}+\boldsymbol{B}_{2}\left(\boldsymbol{B}_{1}^{\prime}+\overline{\boldsymbol{B}}_{1}+m_{1}\right)+n_{1} \boldsymbol{B}_{1}^{\prime}+\overline{\boldsymbol{B}}_{2} m_{1}+\overline{\boldsymbol{B}}_{2} \boldsymbol{B}_{1}^{\prime}} \times Q_{\boldsymbol{B}+\boldsymbol{B}^{\prime}+\overline{\boldsymbol{B}}+\left(3 m_{1}+1,3 n_{1}+1\right)+\left(3 m_{2}+1,3 n_{2}+1\right)} P_{\boldsymbol{B}} P_{\boldsymbol{B}^{\prime}} P_{\overline{\boldsymbol{B}}} \\
&-\sum_{\boldsymbol{B}, \overline{\boldsymbol{B}}, \boldsymbol{B}^{\prime}} q^{n_{2} \boldsymbol{B}_{1}^{\prime}+\left(\boldsymbol{B}_{2}+\overline{\boldsymbol{B}}_{2}+n_{1}\right)} m_{2}+\left(\boldsymbol{B}_{2}+\overline{\boldsymbol{B}}_{2}+n_{1}\right) \boldsymbol{B}_{1}^{\prime}+n_{1} \overline{\boldsymbol{B}}_{1}+\boldsymbol{B}_{2} m_{1}+\boldsymbol{B}_{2} \overline{\boldsymbol{B}}_{1} \\
& \times Q_{\boldsymbol{B}+\boldsymbol{B}^{\prime}+\overline{\boldsymbol{B}}+\left(3 m_{1}+1,3 n_{1}+1\right)+\left(3 m_{2}+1,3 n_{2}+1\right)} P_{\boldsymbol{B}} P_{\boldsymbol{B}^{\prime}} P_{\overline{\boldsymbol{B}}} \\
&+\sum_{\boldsymbol{A}, \boldsymbol{B}, \boldsymbol{B}^{\prime}} q^{n_{1}\left(\boldsymbol{B}_{1}+\boldsymbol{B}_{1}^{\prime}+m_{2}\right)+\boldsymbol{A}_{2} m_{1}+\boldsymbol{A}_{2}\left(\boldsymbol{B}_{1}+\boldsymbol{B}_{1}^{\prime}+m_{2}\right)+n_{2} \boldsymbol{B}_{1}^{\prime}+\boldsymbol{B}_{2} m_{2}+\boldsymbol{B}_{2} \boldsymbol{B}_{1}^{\prime}} \\
& \times Q_{\boldsymbol{A}+\boldsymbol{B}+\boldsymbol{B}^{\prime}+\left(3 m_{1}+1,3 n_{1}+1\right)+\left(3 m_{2}+1,3 n_{2}+1\right)} P_{\boldsymbol{A}} P_{\boldsymbol{B}} P_{\boldsymbol{B}^{\prime}} \\
&+\sum_{\boldsymbol{A}, \boldsymbol{B}, \boldsymbol{B}^{\prime}} q^{n_{1} \boldsymbol{B}_{1}+\left(\boldsymbol{A}_{2}+\boldsymbol{B}_{2}^{\prime}+n_{2}\right) m_{1}+\left(\boldsymbol{A}_{2}+\boldsymbol{B}_{2}^{\prime}+n_{2}\right) \boldsymbol{B}_{1}+n_{2} \boldsymbol{B}_{1}^{\prime}+\boldsymbol{A}_{2} m_{2}+\boldsymbol{A}_{2} \boldsymbol{B}_{1}^{\prime}} \\
& \times Q_{\boldsymbol{A}+\boldsymbol{B}+\boldsymbol{B}^{\prime}+\left(3 m_{1}+1,3 n_{1}+1\right)+\left(3 m_{2}+1,3 n_{2}+1\right)} P_{\boldsymbol{A}} P_{\boldsymbol{B}} P_{\boldsymbol{B}^{\prime}} \\
&-\sum_{\boldsymbol{A}, \boldsymbol{B}, \boldsymbol{B}^{\prime}} q^{n_{2}\left(\boldsymbol{B}_{1}+\boldsymbol{B}_{1}^{\prime}+m_{1}\right)+\boldsymbol{A}_{2} m_{2}+\boldsymbol{A}_{2}\left(\boldsymbol{B}_{1}+\boldsymbol{B}_{1}^{\prime}+m_{1}\right)+n_{1} \boldsymbol{B}_{1}^{\prime}+\boldsymbol{B}_{2} m_{1}+\boldsymbol{B}_{2} \boldsymbol{B}_{1}^{\prime}} \\
& \times Q_{\boldsymbol{A}+\boldsymbol{B}+\boldsymbol{B}^{\prime}+\left(3 m_{1}+1,3 n_{1}+1\right)+\left(3 m_{2}+1,3 n_{2}+1\right)} P_{\boldsymbol{A}} P_{\boldsymbol{B}} P_{\boldsymbol{B}^{\prime}} \\
&-\sum_{\boldsymbol{A}, \boldsymbol{B}, \boldsymbol{B}^{\prime}} q^{n_{2} \boldsymbol{B}_{1}+\left(\boldsymbol{A}_{2}+\boldsymbol{B}_{2}^{\prime}+n_{1}\right) m_{2}+\left(\boldsymbol{A}_{2}+\boldsymbol{B}_{2}^{\prime}+n_{1}\right) \boldsymbol{B}_{1}+n_{1} \boldsymbol{B}_{1}^{\prime}+\boldsymbol{A}_{2} m_{1}+\boldsymbol{A}_{2} \boldsymbol{B}_{1}^{\prime}} \\
& \times Q_{\boldsymbol{A}+\boldsymbol{B}+\boldsymbol{B}^{\prime}+\left(3 m_{1}+1,3 n_{1}+1\right)+\left(3 m_{2}+1,3 n_{2}+1\right)} P_{\boldsymbol{A}} P_{\boldsymbol{B}} P_{\boldsymbol{B}^{\prime}} \\
&=0, \quad
\end{aligned}
$$

where the first two terms cancel, as do the third and the sixth, the fourth and the fifth, and the last two.

$$
\begin{aligned}
& {\left[e^{(\mu)}{ }_{23}\left(m_{1}, n_{1}\right), e^{(\mu)}{ }_{31}\left(m_{2}, n_{2}\right)\right]} \\
& =\left[-q^{-m_{1} n_{1}} \mu P_{-\left(3 m_{1}+1,3 n_{1}+1\right)}-\sum_{\boldsymbol{A}, \boldsymbol{B}} q^{n_{1} \boldsymbol{B}_{1}+\boldsymbol{A}_{2} m_{1}+\boldsymbol{A}_{2} \boldsymbol{B}_{1}} Q_{\boldsymbol{A}+\boldsymbol{B}+\left(3 m_{1}+1,3 n_{1}+1\right)} P_{\boldsymbol{A}} P_{\boldsymbol{B}}\right. \\
& \left.\quad-\sum_{\boldsymbol{B}, \boldsymbol{B}^{\prime}} q^{n_{1} \boldsymbol{B}_{1}^{\prime}+\boldsymbol{B}_{2} m_{1}+\boldsymbol{B}_{2} \boldsymbol{B}_{1}^{\prime}} Q_{\boldsymbol{B}+\boldsymbol{B}^{\prime}+\left(3 m_{1}+1,3 n_{1}+1\right)} P_{\boldsymbol{B}} P_{\boldsymbol{B}^{\prime}}, \sum_{\boldsymbol{A}} q^{\boldsymbol{A}_{1} n_{2}} Q_{\left(3 m_{2}-2,3 n_{2}-2\right)+\boldsymbol{A}} P_{\boldsymbol{A}}\right] \\
& =-q^{-m_{1} n_{1}+\left(-m_{1}-m_{2}\right) n_{2}} \mu P_{-\left(3 m_{1}+1,3 n_{1}+1\right)-\left(3 m_{2}-2,3 n_{2}-2\right)} \\
& -\sum_{\boldsymbol{A}, \boldsymbol{A}^{\prime}} q^{n_{1}\left(m_{2}+\boldsymbol{A}_{1}^{\prime}\right)+\boldsymbol{A}_{2} m_{1}+\boldsymbol{A}_{2}\left(m_{2}+\boldsymbol{A}_{1}^{\prime}\right)+\boldsymbol{A}_{1}^{\prime} n_{2}} Q_{\boldsymbol{A}+\boldsymbol{A}^{\prime}+\left(3 m_{2}-2,3 n_{2}-2\right)+\left(3 m_{1}+1,3 n_{1}+1\right)} P_{\boldsymbol{A}} P_{\boldsymbol{A}^{\prime}} \\
& \quad+\sum_{\boldsymbol{A}, \boldsymbol{B}} q^{\left(\boldsymbol{A}_{1}+\boldsymbol{B}_{1}+m_{1}\right) n_{2}+\boldsymbol{A}_{2} m_{1}+\boldsymbol{A}_{2} \boldsymbol{B}_{1}+n_{1} \boldsymbol{B}_{1}} Q_{\boldsymbol{A}+\boldsymbol{B}+\left(3 m_{2}-2,3 n_{2}-2\right)+\left(3 m_{1}+1,3 n_{1}+1\right)} P_{\boldsymbol{A}} P_{\boldsymbol{B}} \\
& \quad-\sum_{\boldsymbol{A}, \boldsymbol{B}} q^{n_{1}\left(m_{2}+\boldsymbol{A}_{1}\right)+\boldsymbol{B}_{2} m_{1}+\boldsymbol{B}_{2}\left(m_{2}+\boldsymbol{A}_{1}\right)+\boldsymbol{A}_{1} n_{2}} Q_{\boldsymbol{A}+\boldsymbol{B}+\left(3 m_{2}-2,3 n_{2}-2\right)+\left(3 m_{1}+1,3 n_{1}+1\right)} P_{\boldsymbol{A}} P_{\boldsymbol{B}} \\
& \quad-\sum_{\boldsymbol{A}, \boldsymbol{B}} q^{n_{1} \boldsymbol{B}_{1}+\left(\boldsymbol{A}_{2}+n_{2}\right) m_{1}+\left(\boldsymbol{A}_{2}+n_{2}\right) \boldsymbol{B}_{1}+\boldsymbol{A}_{1} n_{2}} Q_{\boldsymbol{A}+\boldsymbol{B}+\left(3 m_{2}-2,3 n_{2}-2\right)+\left(3 m_{1}+1,3 n_{1}+1\right)} P_{\boldsymbol{A}} P_{\boldsymbol{B}}
\end{aligned}
$$

(the third term and the fifth cancel)

$$
\begin{aligned}
=q^{n_{1} m_{2}}( & -q^{-\left(m_{1}+m_{2}\right)\left(n_{1}+n_{2}\right)} \mu P_{-\left(3 m_{1}+3 m_{2}-1,3 n_{1}+3 n_{2}-1\right)} \\
& -\sum_{\boldsymbol{A}, \boldsymbol{A}^{\prime}} q^{\left(n_{1}+n_{2}\right) \boldsymbol{A}_{1}^{\prime}+\boldsymbol{A}_{2}\left(m_{1}+m_{2}\right)+\boldsymbol{A}_{2} \boldsymbol{A}_{1}^{\prime}} Q_{\boldsymbol{A}+\boldsymbol{A}^{\prime}+\left(3 m_{2}+3 m_{1}-1,3 n_{2}+3 n_{1}-1\right)} P_{\boldsymbol{A}} P_{\boldsymbol{A}^{\prime}}
\end{aligned}
$$




$$
\begin{aligned}
& \left.-\sum_{\boldsymbol{A}, \boldsymbol{B}} q^{\left(n_{1}+n_{2}\right) \boldsymbol{A}_{1}+\boldsymbol{B}_{2}\left(m_{1}+m_{2}\right)+\boldsymbol{B}_{2} \boldsymbol{A}_{1}} Q_{\boldsymbol{A}+\boldsymbol{B}+\left(3 m_{2}+3 m_{1}-1,3 n_{2}+3 n_{1}-1\right)} P_{\boldsymbol{A}} P_{\boldsymbol{B}}\right) \\
& =q^{n_{1} m_{2}} e^{(\mu)}{ }_{21}\left(m_{1}+m_{2}, n_{1}+n_{2}\right) \\
& {\left[e^{(\mu)}{ }_{23}\left(m_{1}, n_{1}\right), e^{(\mu)} 32\left(m_{2}, n_{2}\right)\right]} \\
& =\left[-\mu q^{-m_{1} n_{1}} P_{-\left(3 m_{1}+1,3 n_{1}+1\right)}-\sum_{\boldsymbol{A}, \boldsymbol{B}} q^{n_{1} \boldsymbol{B}_{1}+\boldsymbol{A}_{2} m_{1}+\boldsymbol{A}_{2} \boldsymbol{B}_{1}} Q_{\left(3 m_{1}+1,3 n_{1}+1\right)+\boldsymbol{A}+\boldsymbol{B}} P_{\boldsymbol{A}} P_{\boldsymbol{B}}\right. \\
& \left.-\sum_{\boldsymbol{B}, \boldsymbol{B}^{\prime}} q^{n_{1} \boldsymbol{B}_{1}^{\prime}+\boldsymbol{B}_{2} m_{1}+\boldsymbol{B}_{2} \boldsymbol{B}_{1}^{\prime}} Q_{\left(3 m_{1}+1,3 n_{1}+1\right)+\boldsymbol{B}+\boldsymbol{B}^{\prime}} P_{\boldsymbol{B}} P_{\boldsymbol{B}^{\prime}}, Q_{\left(3 m_{2}-1,3 n_{2}-1\right)}\right] \\
& =-\mu q^{-m_{1} n_{1}} \delta_{\left(-m_{1},-n_{1}\right),\left(m_{2}, n_{2}\right)} \\
& -\sum_{\boldsymbol{A}} q^{n_{1} m_{2}+\boldsymbol{A}_{2} m_{1}+\boldsymbol{A}_{2} m_{2}} Q_{\left(3 m_{1}+1,3 n_{1}+1\right)+\boldsymbol{A}+\left(3 m_{2}-1,3 n_{2}-1\right)} P_{\boldsymbol{A}} \\
& -\sum_{\boldsymbol{B}} q^{n_{1} m_{2}+\boldsymbol{B}_{2} m_{1}+\boldsymbol{B}_{2} m_{2}} Q_{\left(3 m_{1}+1,3 n_{1}+1\right)+\boldsymbol{B}+\left(3 m_{2}-1,3 n_{2}-1\right)} P_{\boldsymbol{B}} \\
& -\sum_{\boldsymbol{B}^{\prime}} q^{n_{1} \boldsymbol{B}_{1}^{\prime}+n_{2} m_{1}+n_{2} \boldsymbol{B}_{1}^{\prime}} Q_{\left(3 m_{1}+1,3 n_{1}+1\right)+\boldsymbol{B}^{\prime}+\left(3 m_{2}-1,3 n_{2}-1\right)} P_{\boldsymbol{B}^{\prime}} \\
& =q^{n_{1} m_{2}}\left(-\sum_{\boldsymbol{A}} q^{\boldsymbol{A}_{2}\left(m_{1}+m_{2}\right)} Q_{\left(3 m_{1}+3 m_{2}, 3 n_{1}+3 n_{2}\right)+\boldsymbol{A}} P_{\boldsymbol{A}}\right. \\
& \left.-\sum_{\boldsymbol{B}} q^{\boldsymbol{B}_{2}\left(m_{1}+m_{2}\right)} Q_{\left(3 m_{1}+3 m_{2}, 3 n_{1}+3 n_{2}\right)+\boldsymbol{B}} P_{\boldsymbol{B}}-\frac{1}{2} \mu \delta_{\left(m_{1}+m_{2}, n_{1}+n_{2}\right),(0,0)}\right) \\
& -q^{n_{2} m_{1}}\left(\sum_{\boldsymbol{B}} q^{\boldsymbol{B}_{1}\left(n_{1}+n_{2}\right)} Q_{\left(3 m_{1}+3 m_{2}, 3 n_{1}+3 n_{2}\right)+\boldsymbol{B}} P_{\boldsymbol{B}}+\frac{1}{2} \mu \delta_{\left(m_{1}+m_{2}, n_{1}+n_{2}\right),(0,0)}\right) \\
& =q^{n_{1} m_{2}} e^{(\mu)}{ }_{22}\left(m_{1}+m_{2}, n_{1}+n_{2}\right)-q^{n_{2} m_{1}} e^{(\mu)}{ }_{33}\left(m_{1}+m_{2}, n_{1}+n_{2}\right) \text {. } \\
& {\left[e^{(\mu)}{ }_{23}\left(m_{1}, n_{1}\right), e^{(\mu)} 33\left(m_{2}, n_{2}\right)\right]} \\
& =\left[-\mu q^{-m_{1} n_{1}} P_{-\left(3 m_{1}+1,3 n_{1}+1\right)}-\sum_{\boldsymbol{A}, \boldsymbol{B}} q^{n_{1} \boldsymbol{B}_{1}+\boldsymbol{A}_{2} m_{1}+\boldsymbol{A}_{2} \boldsymbol{B}_{1}} Q_{\left(3 m_{1}+1,3 n_{1}+1\right)+\boldsymbol{A}+\boldsymbol{B}} P_{\boldsymbol{A}} P_{\boldsymbol{B}}\right. \\
& -\sum_{\boldsymbol{B}, \boldsymbol{B}^{\prime}} q^{n_{1} \boldsymbol{B}_{1}^{\prime}+\boldsymbol{B}_{2} m_{1}+\boldsymbol{B}_{2} \boldsymbol{B}_{1}^{\prime}} Q_{\left(3 m_{1}+1,3 n_{1}+1\right)+\boldsymbol{B}+\boldsymbol{B}^{\prime}} P_{\boldsymbol{B}} P_{\boldsymbol{B}^{\prime}}, \\
& \left.\sum_{\boldsymbol{B}} q^{\boldsymbol{B}_{1} n_{2}} Q_{\left(3 m_{2}, 3 n_{2}\right)+\boldsymbol{B}} P_{\boldsymbol{B}}\right] \\
& =-q^{-m_{1} n_{1}+\left(-m_{1}-m-2\right) n_{2}} \mu P_{-\left(3 m_{1}+1,3 n_{1}+1\right)-\left(3 m_{2}, 3 n_{2}\right)} \\
& -\sum_{\boldsymbol{A}, \boldsymbol{B}} q^{n_{1}\left(m_{2}+\boldsymbol{B}_{1}\right)+\boldsymbol{A}_{2} m_{1}+\boldsymbol{B}_{2}\left(m_{2}+\boldsymbol{B}_{1}\right)+\boldsymbol{B}_{1} n_{2}} Q_{\boldsymbol{A}+\boldsymbol{B}+\left(3 m_{1}+1,3 n_{1}+1\right)+\left(3 m_{2}, 3 n_{2}\right)} P_{\boldsymbol{A}} P_{\boldsymbol{B}} \\
& -\sum_{\boldsymbol{B}, \boldsymbol{B}^{\prime}} q^{n_{1}\left(m_{2}+\boldsymbol{B}_{1}^{\prime}\right)+\boldsymbol{B}_{2} m_{1}+\boldsymbol{B}_{2}\left(m_{2}+\boldsymbol{B}_{1}^{\prime}\right)+\boldsymbol{B}_{1}^{\prime} n_{2}} Q_{\boldsymbol{B}+\left(3 m_{2}, 3 n_{2}\right)+\boldsymbol{B}^{\prime}+\left(3 m_{1}+1,3 n_{1}+1\right)} P_{\boldsymbol{B}} P_{\boldsymbol{B}^{\prime}} \\
& -\sum_{\boldsymbol{B}, \boldsymbol{B}^{\prime}} q^{n_{1} \boldsymbol{B}_{1}^{\prime}+\left(n_{2}+\boldsymbol{B}_{2}\right) m_{1}+\left(\boldsymbol{B}_{2}+n_{2}\right) \boldsymbol{B}_{1}^{\prime}+\boldsymbol{B}_{1} n_{2}} Q_{\boldsymbol{B}+\left(3 m_{2}, 3 n_{2}\right)+\boldsymbol{B}^{\prime}+\left(3 m_{1}+1,3 n_{1}+1\right)} P_{\boldsymbol{B}} P_{\boldsymbol{B}^{\prime}} \\
& +\sum_{\boldsymbol{B}, \boldsymbol{B}^{\prime}} q^{\left(\boldsymbol{B}_{1}+\boldsymbol{B}_{1}^{\prime}+m_{1}\right) n_{2}+n_{1} \boldsymbol{B}_{1}^{\prime}+\boldsymbol{B}_{2} m_{1}+\boldsymbol{B}_{2} \boldsymbol{B}_{1}^{\prime}} Q_{\boldsymbol{B}+\left(3 m_{2}, 3 n_{2}\right)+\boldsymbol{B}^{\prime}+\left(3 m_{1}+1,3 n_{1}+1\right)} P_{\boldsymbol{B}} P_{\boldsymbol{B}^{\prime}}
\end{aligned}
$$


(the last two terms cancel)

$$
\begin{aligned}
=q^{n_{1} m_{2}} & -q^{-\left(m_{1}+m_{2}\right)\left(n_{1}+n_{2}\right)} \mu P_{-\left(3 m_{1}+3 m_{2}+1,3 n_{1}+3 n_{2}+1\right)} \\
& -\sum_{\boldsymbol{A}, \boldsymbol{B}} q^{\left(n_{1}+n_{2}\right) \boldsymbol{B}_{1}+\boldsymbol{A}_{2}\left(m_{1}+m_{2}\right)+\boldsymbol{A}_{2} \boldsymbol{B}_{1}} Q_{\boldsymbol{A}+\boldsymbol{B}+\left(3 m_{1}+3 m_{2}+1,3 n_{1}+3 n_{2}+1\right)} P_{\boldsymbol{A}} P_{\boldsymbol{B}} \\
& \left.\quad-\sum_{\boldsymbol{B}, \boldsymbol{B}^{\prime}} q^{\left(n_{1}+n_{2}\right) \boldsymbol{B}_{1}^{\prime}+\boldsymbol{B}_{2}\left(m_{1}+m_{2}\right)+\boldsymbol{B}_{2} \boldsymbol{B}_{1}^{\prime}} Q_{\boldsymbol{B}+\boldsymbol{B}^{\prime}+\left(3 m_{1}+3 m_{2}+1,3 n_{1}+3 n_{2}+1\right)} P_{\boldsymbol{B}} P_{\boldsymbol{B}^{\prime}}\right) \\
= & q^{n_{1} m_{2}} e^{(\mu)}{ }_{23}\left(m_{1}+m_{2}, n_{1}+n_{2}\right) .
\end{aligned}
$$

The next five brackets are easy.

$$
\begin{aligned}
& {\left[e^{(\mu)} 31\left(m_{1}, n_{1}\right), e^{(\mu)} 31\left(m_{2}, n_{2}\right)\right]=0, } \\
& {\left[e^{(\mu)} 31\left(m_{1}, n_{1}\right), e^{(\mu)} 32\left(m_{2}, n_{2}\right)\right]=0, } \\
& {\left[e^{(\mu)} 31\left(m_{1}, n_{1}\right), e^{(\mu)} 33\left(m_{2}, n_{2}\right)\right]=-q^{m_{1} n_{2}} e^{(\mu)}{ }_{31}\left(m_{1}+m_{2}, n_{1}+n_{2}\right), } \\
& {\left[e^{(\mu)} 32\left(m_{1}, n_{1}\right), e^{(\mu)} 32\left(m_{2}, n_{2}\right)\right]=0, } \\
& {\left[e^{(\mu)}{ }_{32}\left(m_{1}, n_{1}\right), e^{(\mu)}{ }_{33}\left(m_{2}, n_{2}\right)\right]=-q^{m_{1} n_{2}} e^{(\mu)}{ }_{32}\left(m_{1}+m_{2}, n_{1}+n_{2}\right) . } \\
& {\left[e^{(\mu)}{ }_{33}\left(m_{1}, n_{1}\right), e^{(\mu)} 33\left(m_{2}, n_{2}\right)\right] }-\sum_{\boldsymbol{B}} q^{\left(\boldsymbol{B}_{1}+m_{1}\right) n_{2}+\boldsymbol{B}_{1} n_{1}} Q_{\left(3 m_{1}, 3 n_{1}\right)+\left(3 m_{2}, 3 n_{2}\right)+\boldsymbol{B}} P_{\boldsymbol{B}} \\
&= {\left[\sum_{\boldsymbol{B}} q^{\boldsymbol{B}_{1} n_{1}} Q_{\left(3 m_{1}, 3 n_{1}\right)+\boldsymbol{B}} P_{\boldsymbol{B}}, \sum_{\boldsymbol{B}} q^{\boldsymbol{B}_{1} n_{2}} Q_{\left(3 m_{2}, 3 n_{2}\right)+\boldsymbol{B}} P_{\boldsymbol{B}}\right] } \\
&= \sum_{\boldsymbol{B}} q^{\left(\boldsymbol{B}_{1}+m_{2}\right) n_{1}+\boldsymbol{B}_{1} n_{2}} Q_{\left(3 m_{1}, 3 n_{1}\right)+\left(3 m_{2}, 3 n_{2}\right)+\boldsymbol{B}} P_{\boldsymbol{B}} \\
&= q^{n_{1} m_{2}} \sum_{\boldsymbol{B}} q^{\boldsymbol{B}_{1}\left(n_{1}+n_{2}\right.} Q_{\left(3 m_{1}, 3 n_{1}\right)+\left(3 m_{2}, 3 n_{2}\right)+\boldsymbol{B}} P_{\boldsymbol{B}} \\
&=-q^{m_{1} n_{2}} \sum_{\boldsymbol{B}} q^{\boldsymbol{B}_{1}\left(n_{1}+n_{2}\right.} Q_{\left(3 m_{1}, 3 n_{1}\right)+\left(3 m_{2}, 3 n_{2}\right)+\boldsymbol{B}} P_{\boldsymbol{B}} \\
& \\
& n_{1} m_{2} e^{(\mu)} 33\left(m_{1}+m_{2}, n_{1}+n_{2}\right)-q^{n_{2} m_{1}} e^{(\mu)} 33\left(m_{1}+m_{2}, n_{1}+n_{2}\right) .
\end{aligned}
$$

Next we check the brackets involving $D_{1}^{(\mu)}$ and $D_{2}^{(\mu)}$.

$$
\begin{aligned}
{\left[D_{1}^{(\mu)}\right.} & \left., e^{(\mu)}{ }_{11}\left(m_{1}, n_{1}\right)\right] \\
& =\left[\sum_{\boldsymbol{A}} \boldsymbol{A}_{1} Q_{\boldsymbol{A}} P_{\boldsymbol{A}}+\sum_{\boldsymbol{B}} \boldsymbol{B}_{1} Q_{\boldsymbol{B}} P_{\boldsymbol{B}}, \sum_{\boldsymbol{A}} q^{\boldsymbol{A}_{1} n_{1}} Q_{\left(3 m_{1}, 3 n_{1}\right)+\boldsymbol{A}} P_{\boldsymbol{A}}+\frac{1}{2} \mu \delta_{\left(m_{1}, n_{1}\right),(0,0)}\right] \\
& =\sum_{\boldsymbol{A}}\left(m_{1}+\boldsymbol{A}_{1}\right) q^{\boldsymbol{A}_{1} n_{1}} Q_{\left(3 m_{1}, 3 n_{1}\right)+\boldsymbol{A}} P_{\boldsymbol{A}}-\sum_{\boldsymbol{A}} \boldsymbol{A}_{1} q^{\boldsymbol{A}_{1} n_{1}} Q_{\left(3 m_{1}, 3 n_{1}\right)+\boldsymbol{A}} P_{\boldsymbol{A}} \\
& =m_{1}\left(\sum_{\boldsymbol{A}} q^{\boldsymbol{A}_{1} n_{1}} Q_{\left(3 m_{1}, 3 n_{1}\right)+\boldsymbol{A}} P_{\boldsymbol{A}}+\frac{1}{2} \mu \delta_{\left(m_{1}, n_{1}\right),(0,0)}\right)=m_{1} e^{(\mu)}{ }_{11}\left(m_{1}, n_{1}\right) .
\end{aligned}
$$

The next two brackets are easy. 


$$
\begin{aligned}
& {\left[D_{1}^{(\mu)}, e^{(\mu)}{ }_{12}\left(m_{1}, n_{1}\right)\right]=m_{1} e^{(\mu)}{ }_{12}\left(m_{1}, n_{1}\right),} \\
& {\left[D_{1}^{(\mu)}, e^{(\mu)}{ }_{13}\left(m_{1}, n_{1}\right)\right]=m_{1} e^{(\mu)}{ }_{13}\left(m_{1}, n_{1}\right) .}
\end{aligned}
$$

$$
\begin{aligned}
& {\left[D_{1}^{(\mu)}, e^{(\mu)}{ }_{21}\left(m_{1}, n_{1}\right)\right]} \\
& =\left[\sum_{\boldsymbol{A}} \boldsymbol{A}_{1} Q_{\boldsymbol{A}} P_{\boldsymbol{A}}+\sum_{\boldsymbol{B}} \boldsymbol{B}_{1} Q_{\boldsymbol{B}} P_{\boldsymbol{B}},\right. \\
& \quad-q^{-m_{1} n_{1}} \mu P_{-\left(3 m_{1}-1,3 n_{1}-1\right)}-\sum_{\boldsymbol{A}, \boldsymbol{A}^{\prime}} q^{n_{1} \boldsymbol{A}_{1}^{\prime}+\boldsymbol{A}_{2} m_{1}+\boldsymbol{A}_{2} \boldsymbol{A}_{1}^{\prime}} Q_{\boldsymbol{A}+\boldsymbol{A}^{\prime}+\left(3 m_{1}-1,3 n_{1}-1\right)} P_{\boldsymbol{A}} P_{\boldsymbol{A}^{\prime}} \\
& \left.\quad-\sum_{\boldsymbol{A}, \boldsymbol{B}} q^{n_{1} \boldsymbol{A}_{1}+\boldsymbol{B}_{2} m_{1}+\boldsymbol{B}_{2} \boldsymbol{A}_{1}} Q_{\boldsymbol{A}+\boldsymbol{B}+\left(3 m_{1}-1,3 n_{1}-1\right)} P_{\boldsymbol{A}} P_{\boldsymbol{B}}\right]
\end{aligned}
$$$$
=q^{-m_{1} n_{1}} \mu\left(-m_{1}\right) P_{-\left(3 m_{1}-1,3 n_{1}-1\right)}
$$$$
-\sum_{\boldsymbol{A}, \boldsymbol{A}^{\prime}}\left(\boldsymbol{A}_{1}+\boldsymbol{A}_{1}^{\prime}+m_{1}\right) q^{n_{1} \boldsymbol{A}_{1}^{\prime}+\boldsymbol{A}_{2} m_{1}+\boldsymbol{A}_{2} \boldsymbol{A}_{1}^{\prime}} Q_{\boldsymbol{A}+\boldsymbol{A}^{\prime}+\left(3 m_{1}-1,3 n_{1}-1\right)} P_{\boldsymbol{A}} P_{\boldsymbol{A}^{\prime}}
$$$$
+\sum_{\boldsymbol{A}, \boldsymbol{A}^{\prime}} \boldsymbol{A}_{1}^{\prime} q^{n_{1} \boldsymbol{A}_{1}^{\prime}+\boldsymbol{A}_{2} m_{1}+\boldsymbol{A}_{2} \boldsymbol{A}_{1}^{\prime}} Q_{\boldsymbol{A}+\boldsymbol{A}^{\prime}+\left(3 m_{1}-1,3 n_{1}-1\right)} P_{\boldsymbol{A}} P_{\boldsymbol{A}^{\prime}}
$$$$
+\sum_{\boldsymbol{A}, \boldsymbol{A}^{\prime}} \boldsymbol{A}_{1} q^{n_{1} \boldsymbol{A}_{1}^{\prime}+\boldsymbol{A}_{2} m_{1}+\boldsymbol{A}_{2} \boldsymbol{A}_{1}^{\prime}} Q_{\boldsymbol{A}+\boldsymbol{A}^{\prime}+\left(3 m_{1}-1,3 n_{1}-1\right)} P_{\boldsymbol{A}} P_{\boldsymbol{A}^{\prime}}
$$$$
-\sum_{\boldsymbol{A}, \boldsymbol{B}}\left(\boldsymbol{A}_{1}+\boldsymbol{B}_{1}+m_{1}\right) q^{n_{1} \boldsymbol{A}_{1}+\boldsymbol{B}_{2} m_{1}+\boldsymbol{B}_{2} \boldsymbol{A}_{1}} Q_{\boldsymbol{A}+\boldsymbol{B}+\left(3 m_{1}-1,3 n_{1}-1\right)} P_{\boldsymbol{A}} P_{\boldsymbol{B}}
$$$$
+\sum_{\boldsymbol{A}, \boldsymbol{B}} \boldsymbol{B}_{1} q^{n_{1} \boldsymbol{A}_{1}+\boldsymbol{B}_{2} m_{1}+\boldsymbol{B}_{2} \boldsymbol{A}_{1}} Q_{\boldsymbol{A}+\boldsymbol{B}+\left(3 m_{1}-1,3 n_{1}-1\right)} P_{\boldsymbol{A}} P_{\boldsymbol{B}}
$$$$
+\sum_{\boldsymbol{A}, \boldsymbol{B}} \boldsymbol{A}_{1} q^{n_{1} \boldsymbol{A}_{1}+\boldsymbol{B}_{2} m_{1}+\boldsymbol{B}_{2} \boldsymbol{A}_{1}} Q_{\boldsymbol{A}+\boldsymbol{B}+\left(3 m_{1}-1,3 n_{1}-1\right)} P_{\boldsymbol{A}} P_{\boldsymbol{B}}
$$$$
=m_{1}\left(-q^{-m_{1} n_{1}} \mu P_{-\left(3 m_{1}-1,3 n_{1}-1\right)}\right.
$$$$
-\sum_{\boldsymbol{A}, \boldsymbol{A}^{\prime}} q^{n_{1} \boldsymbol{A}_{1}^{\prime}+\boldsymbol{A}_{2} m_{1}+\boldsymbol{A}_{2} \boldsymbol{A}_{1}^{\prime}} Q_{\boldsymbol{A}+\boldsymbol{A}^{\prime}+\left(3 m_{1}-1,3 n_{1}-1\right)} P_{\boldsymbol{A}} P_{\boldsymbol{A}^{\prime}}
$$$$
\left.-\sum_{\boldsymbol{A}, \boldsymbol{B}} q^{n_{1} \boldsymbol{A}_{1}+\boldsymbol{B}_{2} m_{1}+\boldsymbol{B}_{2} \boldsymbol{A}_{1}} Q_{\boldsymbol{A}+\boldsymbol{B}+\left(3 m_{1}-1,3 n_{1}-1\right)} P_{\boldsymbol{A}} P_{\boldsymbol{B}}\right)
$$

$=m_{1} e^{(\mu)}{ }_{21}\left(m_{1}, n_{1}\right)$

$\left[D_{1}^{(\mu)}, e^{(\mu)}{ }_{22}\left(m_{1}, n_{1}\right)\right]$

$=\left[\sum_{\boldsymbol{A}} \boldsymbol{A}_{1} Q_{\boldsymbol{A}} P_{\boldsymbol{A}}+\sum_{\boldsymbol{B}} \boldsymbol{B}_{1} Q_{\boldsymbol{B}} P_{\boldsymbol{B}},-\sum_{\boldsymbol{A}} q^{\boldsymbol{A}_{2} m_{1}} Q_{\left(3 m_{1}, 3 n_{1}\right)+\boldsymbol{A}} P_{\boldsymbol{A}}\right.$ $\left.-\sum_{\boldsymbol{B}} q^{\boldsymbol{B}_{2} m_{1}} Q_{\left(3 m_{1}, 3 n_{1}\right)+\boldsymbol{B}} P_{\boldsymbol{B}}-\frac{1}{2} \mu \delta_{\left(m_{1}, n_{1}\right),(0,0)}\right]$

$=-\sum_{\boldsymbol{A}}\left(\boldsymbol{A}_{1}+m_{1}\right) q^{\boldsymbol{A}_{2} m_{1}} Q_{\left(3 m_{1}, 3 n_{1}\right)+\boldsymbol{A}} P_{\boldsymbol{A}}+\sum_{\boldsymbol{A}} \boldsymbol{A}_{1} q^{\boldsymbol{A}_{2} m_{1}} Q_{\left(3 m_{1}, 3 n_{1}\right)+\boldsymbol{A}} P_{\boldsymbol{A}}$

$$
-\sum_{\boldsymbol{B}}\left(m_{1}+\boldsymbol{B}_{1}\right) q^{\boldsymbol{B}_{2} m_{1}} Q_{\left(3 m_{1}, 3 n_{1}\right)+\boldsymbol{B}} P_{\boldsymbol{B}}+\sum_{\boldsymbol{B}} \boldsymbol{B}_{1} q^{\boldsymbol{B}_{2} m_{1}} Q_{\left(3 m_{1}, 3 n_{1}\right)+\boldsymbol{B}} P_{\boldsymbol{B}}
$$


UNITARY REPRESENTATIONS OF THE EXTENDED AFFINE LIE ALGEBRA $\widetilde{\mathfrak{g l}_{3}\left(\mathbb{C}_{q}\right)} 497$

$$
\begin{aligned}
& =m_{1}\left(-\sum_{\boldsymbol{A}} q^{\boldsymbol{A}_{2} m_{1}} Q_{\left(3 m_{1}, 3 n_{1}\right)+\boldsymbol{A}} P_{\boldsymbol{A}}-\sum_{\boldsymbol{B}} q^{\boldsymbol{B}_{2} m_{1}} Q_{\left(3 m_{1}, 3 n_{1}\right)+\boldsymbol{B}} P_{\boldsymbol{B}}-\frac{1}{2} \mu \delta_{\left(m_{1}, n_{1}\right),(0,0)}\right) \\
& =m_{1} e^{(\mu)}{ }_{22}\left(m_{1}, n_{1}\right)
\end{aligned}
$$

$$
\begin{aligned}
& {\left[D_{1}^{(\mu)}, e^{(\mu)}{ }_{23}\left(m_{1}, n_{1}\right)\right]} \\
& =\left[\sum_{\boldsymbol{A}} \boldsymbol{A}_{1} Q_{\boldsymbol{A}} P_{\boldsymbol{A}}+\sum_{\boldsymbol{B}} \boldsymbol{B}_{1} Q_{\boldsymbol{B}} P_{\boldsymbol{B}},\right. \\
& \quad-q^{-m_{1} n_{1}} \mu P_{-\left(3 m_{1}+1,3 n_{1}+1\right)}-\sum_{\boldsymbol{A}, \boldsymbol{B}} q^{n_{1} \boldsymbol{B}_{1}+\boldsymbol{A}_{2} m_{1}+\boldsymbol{A}_{2} \boldsymbol{B}_{1}} Q_{\boldsymbol{A}+\boldsymbol{B}+\left(3 m_{1}+1,3 n_{1}+1\right)} P_{\boldsymbol{A}} P_{\boldsymbol{B}} \\
& \left.\quad-\sum_{\boldsymbol{B}, \boldsymbol{B}^{\prime}} q^{n_{1} \boldsymbol{B}_{1}^{\prime}+\boldsymbol{B}_{2} m_{1}+\boldsymbol{B}_{2} \boldsymbol{B}_{1}^{\prime}} Q_{\boldsymbol{B}+\boldsymbol{B}^{\prime}+\left(3 m_{1}+1,3 n_{1}+1\right)} P_{\boldsymbol{B}} P_{\boldsymbol{B}^{\prime}}\right]
\end{aligned}
$$$$
=q^{-m_{1} n_{1}} \mu\left(-m_{1}\right) P_{-\left(3 m_{1}+1,3 n_{1}+1\right)}
$$$$
-\sum_{\boldsymbol{A}, \boldsymbol{B}}\left(\boldsymbol{A}_{1}+\boldsymbol{B}_{1}+m_{1}\right) q^{n_{1} \boldsymbol{B}_{1}+\boldsymbol{A}_{2} m_{1}+\boldsymbol{A}_{2} \boldsymbol{B}_{1}} Q_{\boldsymbol{A}+\boldsymbol{B}+\left(3 m_{1}+1,3 n_{1}+1\right)} P_{\boldsymbol{A}} P_{\boldsymbol{B}}
$$$$
+\sum_{\boldsymbol{A}, \boldsymbol{B}} \boldsymbol{A}_{1} q^{n_{1} \boldsymbol{B}_{1}+\boldsymbol{A}_{2} m_{1}+\boldsymbol{A}_{2} \boldsymbol{B}_{1}} Q_{\boldsymbol{A}+\boldsymbol{B}+\left(3 m_{1}+1,3 n_{1}+1\right)} P_{\boldsymbol{A}} P_{\boldsymbol{B}}
$$$$
+\sum_{\boldsymbol{A}, \boldsymbol{B}} \boldsymbol{B}_{1} q^{n_{1} \boldsymbol{B}_{1}+\boldsymbol{A}_{2} m_{1}+\boldsymbol{A}_{2} \boldsymbol{B}_{1}} Q_{\boldsymbol{A}+\boldsymbol{B}+\left(3 m_{1}+1,3 n_{1}+1\right)} P_{\boldsymbol{A}} P_{\boldsymbol{B}}
$$$$
-\sum_{\boldsymbol{B}, \boldsymbol{B}^{\prime}}\left(\boldsymbol{B}_{1}+\boldsymbol{B}_{1}^{\prime}+m_{1}\right) q^{n_{1} \boldsymbol{B}_{1}^{\prime}+\boldsymbol{B}_{2} m_{1}+\boldsymbol{B}_{2} \boldsymbol{B}_{1}^{\prime}} Q_{\boldsymbol{B}+\boldsymbol{B}^{\prime}+\left(3 m_{1}+1,3 n_{1}+1\right)} P_{\boldsymbol{B}} P_{\boldsymbol{B}^{\prime}}
$$$$
+\sum_{\boldsymbol{B}, \boldsymbol{B}^{\prime}} \boldsymbol{B}_{1} q^{n_{1} \boldsymbol{B}_{1}^{\prime}+\boldsymbol{B}_{2} m_{1}+\boldsymbol{B}_{2} \boldsymbol{B}_{1}^{\prime}} Q_{\boldsymbol{B}+\boldsymbol{B}^{\prime}+\left(3 m_{1}+1,3 n_{1}+1\right)} P_{\boldsymbol{B}} P_{\boldsymbol{B}^{\prime}}
$$$$
+\sum_{\boldsymbol{B}, \boldsymbol{B}^{\prime}} \boldsymbol{B}_{1}^{\prime} q^{n_{1} \boldsymbol{B}_{1}^{\prime}+\boldsymbol{B}_{2} m_{1}+\boldsymbol{B}_{2} \boldsymbol{B}_{1}^{\prime}} Q_{\boldsymbol{B}+\boldsymbol{B}^{\prime}+\left(3 m_{1}+1,3 n_{1}+1\right)} P_{\boldsymbol{B}} P_{\boldsymbol{B}^{\prime}}
$$$$
=m_{1}\left(-q^{-m_{1} n_{1}} \mu P_{-\left(3 m_{1}+1,3 n_{1}+1\right)}\right.
$$$$
-\sum_{\boldsymbol{A}, \boldsymbol{B}} q^{n_{1} \boldsymbol{B}_{1}+\boldsymbol{A}_{2} m_{1}+\boldsymbol{A}_{2} \boldsymbol{B}_{1}} Q_{\boldsymbol{A}+\boldsymbol{B}+\left(3 m_{1}+1,3 n_{1}+1\right)} P_{\boldsymbol{A}} P_{\boldsymbol{B}}
$$$$
\left.-\sum_{\boldsymbol{B}, \boldsymbol{B}^{\prime}} q^{n_{1} \boldsymbol{B}_{1}^{\prime}+\boldsymbol{B}_{2} m_{1}+\boldsymbol{B}_{2} \boldsymbol{B}_{1}^{\prime}} Q_{\boldsymbol{B}+\boldsymbol{B}^{\prime}+\left(3 m_{1}+1,3 n_{1}+1\right)} P_{\boldsymbol{B}} P_{\boldsymbol{B}^{\prime}}\right)
$$

$$
=m_{1} e^{(\mu)}{ }_{23}\left(m_{1}, n_{1}\right) \text {. }
$$

The following two brackets are easy.

$$
\begin{aligned}
& {\left[D_{1}^{(\mu)}, e^{(\mu)}{ }_{31}\left(m_{1}, n_{1}\right)\right]=m_{1} e^{(\mu)}{ }_{31}\left(m_{1}, n_{1}\right),} \\
& {\left[D_{1}^{(\mu)}, e^{(\mu)}{ }_{32}\left(m_{1}, n_{1}\right)\right]=m_{1} e^{(\mu)}{ }_{32}\left(m_{1}, n_{1}\right) .}
\end{aligned}
$$

$$
\begin{aligned}
& {\left[D_{1}^{(\mu)}, e^{(\mu)}{ }_{33}\left(m_{1}, n_{1}\right)\right]} \\
& \quad=\left[\sum_{\boldsymbol{A}} \boldsymbol{A}_{1} Q_{\boldsymbol{A}} P_{\boldsymbol{A}}+\sum_{\boldsymbol{B}} \boldsymbol{B}_{1} Q_{\boldsymbol{B}} P_{\boldsymbol{B}}, \sum_{\boldsymbol{B}} q^{\boldsymbol{B}_{1} n_{1}} Q_{\left(3 m_{1}, 3 n_{1}\right)+\boldsymbol{B}} P_{\boldsymbol{B}}+\frac{1}{2} \mu \delta_{\left(m_{1}, n_{1}\right),(0,0)}\right]
\end{aligned}
$$




$$
\begin{aligned}
& =\sum_{\boldsymbol{B}}\left(m_{1}+\boldsymbol{B}_{1}\right) q^{\boldsymbol{B}_{1} n_{1}} Q_{\left(3 m_{1}, 3 n_{1}\right)+\boldsymbol{B}} P_{\boldsymbol{B}}-\sum_{\boldsymbol{B}} \boldsymbol{B}_{1} q^{\boldsymbol{B}_{1} n_{1}} Q_{\left(3 m_{1}, 3 n_{1}\right)+\boldsymbol{B}} P_{\boldsymbol{B}} \\
& =m_{1}\left(\sum_{\boldsymbol{B}} q^{\boldsymbol{B}_{1} n_{1}} Q_{\left(3 m_{1}, 3 n_{1}\right)+\boldsymbol{B}} P_{\boldsymbol{B}}+\frac{1}{2} \mu \delta_{\left(m_{1}, n_{1}\right),(0,0)}\right) \\
& =m_{1} e^{(\mu)}{ }_{33}\left(m_{1}, n_{1}\right) .
\end{aligned}
$$

Similarly, we can get

$$
\left[D_{2}^{(\mu)}, e^{(\mu)}{ }_{i j}\left(m_{1}, n_{1}\right)\right]=n_{1} e^{(\mu)}{ }_{i j}\left(m_{1}, n_{1}\right)
$$

for $1 \leq i, j \leq 3$. Finally,

$$
\left[D_{1}^{(\mu)}, D_{2}^{(\mu)}\right]=\left[\sum_{\boldsymbol{A}} \boldsymbol{A}_{1} Q_{\boldsymbol{A}} P_{\boldsymbol{A}}+\sum_{\boldsymbol{B}} \boldsymbol{B}_{1} Q_{\boldsymbol{B}} P_{\boldsymbol{B}}, \sum_{\boldsymbol{A}} \boldsymbol{A}_{2} Q_{\boldsymbol{A}} P_{\boldsymbol{A}}+\sum_{\boldsymbol{B}} \boldsymbol{B}_{2} Q_{\boldsymbol{B}} P_{\boldsymbol{B}}\right]=0 .
$$

Hence $\pi: \widetilde{\mathfrak{g l}_{3}\left(\mathbb{C}_{q}\right)} \rightarrow \operatorname{End}(V)$ is a Lie algebra homomorphism.

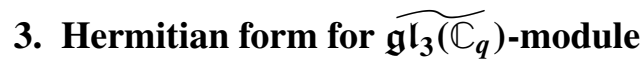

From now on we need to assume that $|q|=1$.

Define a $\mathbb{R}$-linear map $\omega: \overline{\mathfrak{g l}_{3}\left(\mathbb{C}_{q}\right)} \mapsto \widehat{\mathfrak{g l}_{3}\left(\mathbb{C}_{q}\right)}$ by

$$
\begin{aligned}
\omega(\lambda x) & =\bar{\lambda} \omega(x) \quad \text { for all } \lambda \in \mathbb{C} \text { and } x \in \widetilde{\mathfrak{g l}_{3}\left(\mathbb{C}_{q}\right)}, \\
\omega\left(E_{i j}(a)\right)=(-1)^{i+j} E_{j i}(\bar{a}) & \text { for } a \in \mathbb{C}_{q}, \\
& \omega\left(d_{s}\right)=d_{s}, \quad \omega\left(c_{s}\right)=c_{s}, \\
& \omega\left(d_{t}\right)=d_{t}, \quad \omega\left(c_{t}\right)=c_{t} .
\end{aligned}
$$

Here, the $\mathbb{R}$-linear function $-: \mathbb{C}_{q} \rightarrow \mathbb{C}_{q}$ is defined as $\overline{\lambda s^{m} t^{n}}=\bar{\lambda} t^{-n} s^{-m}=$ $\bar{\lambda} q^{m n} s^{-m} t^{-n}$, where $\bar{\lambda}$ is the complex conjugate for any $\lambda \in \mathbb{C}$ and $m, n \in \mathbb{Z}$.

From [Gao and Zeng 2006, Lemma 3.4], we have

Lemma 3.1. $\omega$ is an antilinear antiinvolution of $\widetilde{\mathfrak{g l}_{3}\left(\mathbb{C}_{q}\right)}$.

We write $\pi\left(E_{i j}(r)\right) \cdot v$ more simply as $E_{i j}(r) \cdot v$, for any $v \in V, r \in \mathbb{C}_{q}$.

In [Gao and Zeng 2006], we defined a hermitian form on the basis consisting of monomials and then used another basis consisting of iterated module actions on the "highest weight vector" 1 to determine the condition for the form being positive definite. Here we will use the second basis directly to define the hermitian form which is much simpler.

Lemma 3.2. Consider, for $k, l \in \mathbb{Z}_{+} \cup\{0\}$, the "vectors"

$$
E_{12}\left(\alpha_{1}\right) E_{12}\left(\alpha_{2}\right) \cdots E_{12}\left(\alpha_{k}\right) E_{32}\left(\beta_{1}\right) E_{32}\left(\beta_{2}\right) \cdots E_{32}\left(\beta_{l}\right) \cdot 1,
$$


where

$$
\begin{array}{ll}
\alpha_{i}=s^{m_{i}} t^{n_{i}} & \text { for } i=1, \ldots, k, \\
\beta_{j}=s^{u_{j}} t^{v_{j}} & \text { for } j=1, \ldots, l,
\end{array}
$$

and $m_{i}, n_{i}, u_{j}, v_{j} \in \mathbb{Z}$. We say each is in level $(k, l)$ in $W$, and together they form a basis for $V$.

Proof. Define

$$
f_{\boldsymbol{A}, \boldsymbol{B}}=\prod_{(m, n) \in \mathbb{Z}^{2}} x_{(3 m+1,3 n+1)}^{\boldsymbol{A}_{(m, n)}} \cdot \prod_{\left(m^{\prime}, n^{\prime}\right) \in \mathbb{Z}^{2}} x_{\left(3 m^{\prime}-1,3 n^{\prime}-1\right)}^{\boldsymbol{B}_{\left(r^{\prime}, n^{\prime}\right)}},
$$

for $\boldsymbol{A}_{(m, n)}, \boldsymbol{B}_{\left(m^{\prime}, n^{\prime}\right)} \in \mathbb{Z}_{+} \cup\{0\}$, where only finitely many $\boldsymbol{A}_{(m, n)}, \boldsymbol{B}_{\left(m^{\prime}, n^{\prime}\right)}$ are nonzero. The $f_{\boldsymbol{A}, \boldsymbol{B}}$ form a basis for $V$.

Let

$$
g_{\boldsymbol{A}}=\prod_{(m, n) \in \mathbb{Z}^{2}} x_{(3 m+1,3 n+1)}^{\boldsymbol{A}_{(m, n)}} \quad \text { and } \quad h_{\boldsymbol{B}}=\prod_{\left(m^{\prime}, n^{\prime}\right) \in \mathbb{Z}^{2}} x_{\left(3 m^{\prime}-1,3 n^{\prime}-1\right)}^{\boldsymbol{B}_{\left(m^{\prime}, n^{\prime}\right)}}
$$

In a way similar to [Gao and Zeng 2006, Lemma 4.2], we can write $g_{A}$ as a linear combination of $E_{12}\left(\alpha_{1}\right) \cdots E_{12}\left(\alpha_{k}\right) \cdot 1$ for $k \leq \sum_{(m, n)} \boldsymbol{A}_{(m, n)}$, and $h_{\boldsymbol{B}}$ can be written as a linear combination of $E_{32}\left(\beta_{1}\right) \cdots E_{32}\left(\beta_{l}\right) \cdot 1$ for $l \leq \sum_{\left(m^{\prime}, n^{\prime}\right)} \boldsymbol{B}_{\left(m^{\prime}, n^{\prime}\right)}$.

Since $E_{12}(\alpha) E_{32}(\beta) \cdot u=E_{32}(\beta) E_{12}(\alpha) \cdot u$ for any $u \in V$, we can write $f_{(\boldsymbol{A}, \boldsymbol{B})}$ as a linear combination of $E_{12}\left(\alpha_{1}\right) \cdots E_{12}\left(\alpha_{k}\right) E_{32}\left(\beta_{1}\right), \ldots, E_{32}\left(\beta_{l}\right) \cdot 1$. Hence the collection of $E_{12}\left(\alpha_{1}\right) \cdots E_{12}\left(\alpha_{k}\right) E_{32}\left(\beta_{1}\right), \ldots, E_{32}\left(\beta_{l}\right) \cdot 1$ form a basis for $V$.

Denote this basis in $V$ by

$$
\mathscr{B}=\left\{E_{12}\left(\alpha_{1}\right) \cdots E_{12}\left(\alpha_{k}\right) E_{32}\left(\beta_{1}\right) \cdots E_{32}\left(\beta_{l}\right) \cdot 1 \mid \text { for all } k, l \in \mathbb{N}, \alpha_{i}, \beta_{j} \in \mathbb{C}_{q}\right\} .
$$

Lemma 3.3. For any $v \in V$,

- $\operatorname{lev}(v)=\operatorname{lev}\left(E_{i i}(a) \cdot v\right)$ for $i=1,2,3$;

- $\operatorname{lev}\left(E_{12}(a)(v)\right)=\operatorname{lev}(v)+(1,0)$;

- $\operatorname{lev}\left(E_{32}(a) \cdot v\right)=\operatorname{lev}(v)+(0,1) ;$

- $\operatorname{lev}\left(E_{21}(a) \cdot v\right)=\operatorname{lev}(v)-(1,0)$ or $E_{21}(a) \cdot v=0$ if $\operatorname{lev}(v)-(1,0) \notin \mathbb{Z}_{+}^{2}$;

- $\operatorname{lev}\left(E_{23}(a) \cdot v\right)=\operatorname{lev}(v)-(0,1)$ or $E_{23}(a) \cdot v=0$ if $\operatorname{lev}(v)-(0,1) \notin \mathbb{Z}_{+}^{2}$,

for any nonzero $a \in \mathbb{C}_{q}$.

Proof. We only check those $v$ in the basis $\mathscr{B}$.

$$
\begin{aligned}
& E_{22}(a) E_{12}\left(\alpha_{1}\right) E_{12}\left(\alpha_{2}\right) \cdots E_{12}\left(\alpha_{k}\right) E_{32}\left(\beta_{1}\right) E_{32}\left(\beta_{2}\right) \cdots E_{32}\left(\beta_{l}\right) \cdot 1 \\
& =E_{12}\left(\alpha_{1}\right) E_{22}(a) E_{12}\left(\alpha_{2}\right) \cdots E_{12}\left(\alpha_{k}\right) E_{32}\left(\beta_{1}\right) E_{32}\left(\beta_{2}\right) \cdots E_{32}\left(\beta_{l}\right) \cdot 1 \\
& \quad-E_{12}\left(\alpha_{1} a\right) E_{12}\left(\alpha_{2}\right) \cdots E_{12}\left(\alpha_{k}\right) E_{32}\left(\beta_{1}\right) E_{32}\left(\beta_{2}\right) \cdots E_{32}\left(\beta_{l}\right) \cdot 1 \\
& =E_{12}\left(\alpha_{1}\right) E_{12}\left(\alpha_{2}\right) \cdots E_{12}\left(\alpha_{k}\right) E_{32}\left(\beta_{1}\right) E_{32}\left(\beta_{2}\right) \cdots E_{32}\left(\beta_{l}\right) \cdot\left(\frac{1}{2} \mu\right) \kappa(a) \cdot 1
\end{aligned}
$$




$$
\begin{aligned}
& -\sum_{i=1}^{k} E_{12}\left(\alpha_{1}\right) \cdots E_{12}\left(\alpha_{i} a\right) \cdots E_{12}\left(\alpha_{k}\right) E_{32}\left(\beta_{1}\right) E_{32}\left(\beta_{2}\right) \cdots E_{32}\left(\beta_{l}\right) \cdot 1 \\
& +\sum_{i=1}^{l} E_{12}\left(\alpha_{1}\right) E_{12}\left(\alpha_{2}\right) \cdots E_{12}\left(\alpha_{k}\right) E_{32}\left(\beta_{1}\right) \cdots E_{32}\left(\beta_{i} a\right) \cdots E_{32}\left(\beta_{l}\right) \cdot 1
\end{aligned}
$$

so $\operatorname{lev}(v)=\operatorname{lev}\left(E_{22}(a) \cdot v\right)$. It is similar for $E_{11}(a), E_{33}(a)$.

Further, $\operatorname{lev}\left(E_{12}(a)(v)\right)=\operatorname{lev}(v)+(1,0)$ and $\operatorname{lev}\left(E_{32}(a) \cdot v\right)=\operatorname{lev}(v)+(0,1)$ are the definition of level.

For $E_{21}(a) . v$, we prove by induction on the level of $v: E_{21}(a) \cdot v=0$ if $\operatorname{lev}(v)=$ $(0, n), n \in \mathbb{Z}_{+} \cup\{0\}$. If $n=0$, it is obvious that $E_{21}(a) \cdot 1=0$. Suppose it is true for $n$, then

$$
\begin{aligned}
& E_{21}(a) E_{32}\left(\beta_{1}\right) E_{32}\left(\beta_{2}\right) \cdots E_{32}\left(\beta_{n+1}\right) \cdot 1 \\
& \quad=E_{32}\left(\beta_{1}\right) E_{21}(a) E_{32}\left(\beta_{2}\right) \cdots E_{32}\left(\beta_{n+1}\right) \cdot 1-E_{31}\left(\beta_{1} a\right) E_{32}\left(\beta_{2}\right) \cdots E_{32}\left(\beta_{n+1}\right) \cdot 1 \\
& \quad=-E_{32}\left(\beta_{2}\right) \cdots E_{32}\left(\beta_{n+1}\right) E_{31}\left(\beta_{1} a\right) \cdot 1 \\
& \quad=0
\end{aligned}
$$

by induction.

Supposing $\operatorname{lev}\left(E_{21}(a) \cdot v\right)=\operatorname{lev}(v)-(1,0)$ or $E_{21}(a) \cdot v=0$ is true for $\operatorname{lev}(v)=$ $(m-1, n)$, then for $v=E_{12}(b) v^{\prime}$ with $\operatorname{lev}\left(v^{\prime}\right)=(m-1, n)$ and $0 \neq b \in \mathbb{C}_{q}$, we have

$$
E_{21}(a) \cdot E_{12}(b) \cdot v^{\prime}=E_{12}(b) E_{21}(a) \cdot v^{\prime}+E_{22}(a b) \cdot v^{\prime}-E_{11}(b a) \cdot v^{\prime} .
$$

Since $\operatorname{lev}\left(E_{21}(a) \cdot v^{\prime}\right)=(m-2, n)$, we have $\operatorname{lev}\left(E_{21}(a) \cdot E_{12}(b) \cdot v^{\prime}\right)=(m-1, n)$ or $E_{21}(a) \cdot E_{12}(b) \cdot v^{\prime}=0$. It is similar for $E_{23}(a)$.

We easily define a contravariant (with respect to $\pi, \omega$ ) hermitian form on $V$ by using the basis $\mathscr{B}$.

Assuming that $\mu$ is a real number, define the conjugate bilinear form on the elements in $\mathscr{B}$ by induction on the level:

$$
(1,1)=1,(1, f)=0 \quad \text { if } \quad \operatorname{lev}(f) \neq(0,0)
$$

Suppose for any $v \in \mathscr{B},(u, v)$ is defined for any $u$ such that lev $(u)=\left(k^{\prime}, l^{\prime}\right)$ with $k^{\prime}+l^{\prime}=r-1$ if $\operatorname{lev}(u)=(k, l)$, with $k+l=r$. Then there exists a $u^{\prime}$ such that $\operatorname{lev}\left(u^{\prime}\right)=(k-1, l)$ or $\operatorname{lev}\left(u^{\prime}\right)=(k, l-1)$ and some $a \in \mathbb{C}_{q}$ such that $u=E_{12}(a) \cdot u^{\prime}$ or $u=E_{32}(a) \cdot u^{\prime}$.

Theorem 3.4. The conjugate bilinear form defined through

$$
\left(E_{12}(a) \cdot u^{\prime}, v\right)=\left(u^{\prime}, \omega\left(E_{12}(a)\right) \cdot v\right) \quad \text { and } \quad\left(E_{32}(a) \cdot u^{\prime}, v\right)=\left(u^{\prime}, \omega\left(E_{32}(a)\right) \cdot v\right)
$$

is a hermitian form on $V$. 
Proof. We must check that $\left(E_{i j}(a) \cdot u, v\right)=\left(u, \omega\left(E_{i j}(a)\right) \cdot v\right)$ for $1 \leq i, j \leq 3$, and $a \in \mathbb{C}_{q}$. We must also check $\left(D_{i} \cdot u, v\right)=\left(u, \omega\left(D_{i}\right) \cdot v\right)$ for $i=1,2$.

By definition,

$$
\left(E_{12}(a) u, v\right)=\left(u, \omega\left(E_{12}(a)\right) v\right) \quad \text { and } \quad\left(E_{32}(a) u, v\right)=\left(u, \omega\left(E_{32}(a)\right) v\right),
$$

and so

$$
\begin{aligned}
\left(E_{13}(a) u, v\right) & =\left(\left[E_{12}(1), E_{23}(a)\right] u, v\right) \\
& =\left(E_{12}(1) E_{23}(a) u, v\right)-\left(E_{23}(a) E_{12}(1) u, v\right) \\
& =\left(u, \omega\left(E_{23}(a)\right) \omega\left(E_{12}(1)\right) v\right)-\left(u, \omega\left(E_{12}(1)\right) \omega\left(E_{23}(a)\right) v\right) \\
& =\left(u,-\omega\left(\left[E_{23}(a), E_{12}(1)\right]\right) v\right)=\left(u, \omega\left(E_{13}(a)\right) v\right) .
\end{aligned}
$$

We use induction on $\operatorname{lev}(u)$ to prove $\left(E_{11}(a) \cdot u, v\right)=\left(u, \omega\left(E_{11}(a)\right) \cdot v\right)$. For any $v \in \mathscr{B}$,

$$
\left(E_{11}(a) 1, v\right)=\frac{1}{2} \mu \kappa(a)(1, v)=\frac{1}{2} \mu \kappa(a) \delta_{1, v} .
$$

Since $\operatorname{lev}\left(E_{11}(a) \cdot v\right)=\operatorname{lev}(v)$ for any $v \in \mathscr{B}$,

$$
\left(1, \omega\left(E_{11}(a)\right) \cdot v\right)=\left(1, E_{11}(\bar{a}) \cdot v\right)=\frac{1}{2} \mu \kappa(\bar{a}) \delta_{1, v} .
$$

Hence

$$
\left(E_{11}(a) \cdot 1, v\right)=\left(1, \omega\left(E_{11}(a)\right) \cdot v\right) .
$$

Suppose $\left(E_{11}(a) \cdot u, v\right)=\left(u, \omega\left(E_{11}(a)\right) \cdot v\right)$ holds true for any $\operatorname{lev}(u)=(l, k)$ with $l+k=r-1$. Then for $\operatorname{lev}(u)=(l, k)$ with $l+k=r$, we have $u=E_{32}(b) \cdot u^{\prime}$, with $\operatorname{lev}\left(u^{\prime}\right)=(l, k-1)$, and

$$
\begin{aligned}
\left(E_{11}(a) E_{32}(b) \cdot u^{\prime}, v\right) & =\left(E_{32}(b) E_{11}(a) \cdot u^{\prime}, v\right)=\left(E_{11}(a) \cdot u^{\prime}, \omega\left(E_{32}(b)\right) \cdot v\right) \\
& =\left(u^{\prime}, \omega\left(E_{11}(a)\right) \omega\left(E_{32}(b)\right) \cdot v\right) \\
& =\left(u^{\prime}, \omega\left(E_{32}(b)\right) \omega\left(E_{11}(a)\right) \cdot v\right) \\
& =\left(E_{32}(b) \cdot u^{\prime}, \omega\left(E_{11}(a)\right) \cdot v\right)=\left(u, \omega\left(E_{11}(a)\right) \cdot v\right),
\end{aligned}
$$

or $u=E_{12}(b) \cdot u^{\prime}$, with $\operatorname{lev}\left(u^{\prime}\right)=(l-1, k)$, and

$$
\begin{aligned}
\left(E_{11}(a) E_{12}(b)\right. & \left.\cdot u^{\prime}, v\right) \\
& =\left(E_{12}(b) E_{11}(a) \cdot u^{\prime}, v\right)+\left(\left[E_{11}(a), E_{12}(b)\right] \cdot u^{\prime}, v\right) \\
& =\left(E_{11}(a) \cdot u^{\prime}, \omega\left(E_{12}(b)\right) \cdot v\right)+\left(u^{\prime}, \omega\left(\left[E_{11}(a), E_{12}(b)\right]\right) \cdot v\right) \\
& =\left(u^{\prime}, \omega\left(E_{11}(a)\right) \omega\left(E_{12}(b)\right) \cdot v\right)-\left(u^{\prime},\left[\omega\left(E_{11}(a)\right), \omega\left(E_{12}(b)\right)\right] \cdot v\right) \\
& =\left(u^{\prime}, \omega\left(E_{12}(b)\right) \omega\left(E_{11}(a)\right) \cdot v\right) \\
& =\left(E_{12}(b) u^{\prime}, \omega\left(E_{11}(a)\right) \cdot v\right)=\left(u, \omega\left(E_{11}(a)\right) \cdot v\right) .
\end{aligned}
$$


Thus $\left(E_{11}(a) \cdot u, v\right)=\left(u, \omega\left(E_{11}(a)\right) \cdot v\right)$; and

$$
\begin{aligned}
\left(E_{22}(a) \cdot u, v\right)= & \left(\left[E_{21}(a), E_{12}(1)\right] \cdot u, v\right)+\left(E_{11}(a) u, v\right) \\
= & \left(E_{21}(a) E_{12}(1) \cdot u, v\right)-\left(E_{12}(1) E_{21}(a) \cdot u, v\right)+\left(E_{11}(a) u, v\right) \\
= & \left(u, \omega\left(E_{12}(1)\right) \omega\left(E_{21}(a)\right) \cdot v\right) \\
& \quad-\left(u, \omega\left(E_{21}(a)\right) \omega\left(E_{12}(1)\right) \cdot v\right)+\left(u, \omega\left(E_{11}(a)\right) \cdot v\right) \\
= & \left(u, \omega\left(\left[E_{21}(a), E_{12}(1)\right] \cdot v\right)\right)+\left(u, \omega\left(E_{11}(a)\right) \cdot v\right) \\
= & \left(u, \omega\left(E_{22}(a)\right) \cdot v\right) .
\end{aligned}
$$

It is similar for $\left(E_{33}(a) \cdot u, v\right)=\left(u, \omega\left(E_{33}(a)\right) \cdot v\right)$.

For $D_{1}, D_{2}$, we also proceed by induction on the level of $u$. It is obvious that $\left(D_{1} \cdot 1, v\right)=0$ for any $v \in \mathscr{B}$, and so $\left(D_{1} \cdot 1,1\right)=\left(1, D_{1} \cdot 1\right)=0$. Suppose $\left(1, D_{1} \cdot v\right)=0$ is true for those $\operatorname{lev}(v)=\left(k^{\prime}, l^{\prime}\right)$ with $k^{\prime}+l^{\prime}=r>0$. Then

$$
\begin{aligned}
& \left(1, D_{1} E_{12}\left(s^{m} t^{n}\right) \cdot v\right)=\left(1, E_{12} D_{1} \cdot v\right)+(1, m \cdot v)=0, \\
& \left(1, D_{1} E_{32}\left(s^{m} t^{n}\right) \cdot v\right)=\left(1, E_{32} D_{1} \cdot v\right)+(1, m \cdot v)=0 .
\end{aligned}
$$

Thus $\left(D_{1} \cdot 1, v\right)=\left(1, D_{1} \cdot v\right)$.

Suppose for any $v \in \mathscr{B}$ that $\left(D_{1} \cdot u, v\right)=\left(u, D_{1} \cdot v\right)$ is true for all $\operatorname{lev}(u)=\left(k^{\prime}, l^{\prime}\right)$ such that $k^{\prime}+l^{\prime}=r$, then

$$
\begin{aligned}
\left(D_{1} \cdot E_{12}\left(s^{m} t^{n}\right) \cdot u, v\right) & =\left(E_{12}\left(s^{m} t^{n}\right) D_{1} \cdot u, v\right)+(m \cdot u, v) \\
& =\left(D_{1} \cdot u, \omega\left(E_{12}\left(s^{m} t^{n}\right)\right) \cdot v\right)+(u, m \cdot v) \\
& =\left(u, D_{1} \omega\left(E_{12}\left(s^{m} t^{n}\right)\right) \cdot v\right)+(u, m \cdot v) \\
& =\left(u, \omega\left(E_{12}\left(s^{m} t^{n}\right)\right) D_{1} \cdot v\right) \\
& =\left(E_{12}\left(s^{m} t^{n}\right) u, D_{1} \cdot v\right) .
\end{aligned}
$$

It is similar for $\left(D_{1} \cdot E_{32}\left(s^{m} t^{n}\right) \cdot u, v\right)=\left(E_{32}\left(s^{m} t^{n}\right) \cdot u, D_{1} \cdot v\right)$.

Hence $\left(D_{1} \cdot u, v\right)=\left(u, D_{1} \cdot v\right)$, and $\left(D_{2} \cdot u, v\right)=\left(u, D_{2} \cdot v\right)$. Note that $\omega\left(D_{i}\right)=D_{i}$ for $i=1,2$.

\section{Conditions for unitarity}

In this section we will determine when the hermitian form given last section is positive definite.

Let $i \in \mathbb{N}, \gamma=\left(\gamma_{1}, \ldots, \gamma_{s}\right)$ be the $s$-partition of $i$. We denote by $\operatorname{Par}_{s}(i)$ be the set of all $s$-partition of $i$.

Let $\gamma \in \operatorname{Par}_{s}(N)$. We say $p i_{1}^{\prime} \times \pi_{2}^{\prime} \in S_{N} \times S_{N}$ is equivalent to $\pi_{1} \times \pi_{2} \in S_{N} \times S_{N}$, where $S_{N}$ is the permutation group of $N$ letters, if, for all $z_{1}, \ldots, z_{N} \in \mathbb{C}_{q}$ and 
UNITARY REPRESENTATIONS OF THE EXTENDED AFFINE LIE ALGEBRA $\widetilde{\mathfrak{g l}_{3}\left(\mathbb{C}_{q}\right)} 503$

$$
\begin{aligned}
& w_{1}, \ldots, w_{N} \in \mathbb{C}_{q}, \\
& \kappa\left(z_{\pi_{1}^{\prime}(1)} w_{\pi_{2}^{\prime}(1)}, \ldots, z_{\pi_{1}^{\prime}\left(\gamma_{1}\right)} w_{\pi_{2}^{\prime}\left(\gamma_{1}\right)}\right) \ldots \\
& \quad \kappa\left(z_{\pi_{1}^{\prime}\left(\gamma_{1}+, \ldots, \gamma_{s-1}+1\right)} w_{\pi_{2}^{\prime}\left(\gamma_{1}+, \ldots,+\gamma_{s-1}+1\right)}, \ldots, z_{\pi_{1}^{\prime}(N)} w_{\pi_{2}^{\prime}(N)}\right)
\end{aligned}
$$

can be obtained from the analogous expression for $\pi_{1} \times \pi_{2}$ by only rotating the variables. For example, $\kappa\left(z_{1} w_{1} z_{2} w_{2} z_{3} w_{3}\right)=\kappa\left(z_{3} w_{3} z_{1} w_{1} z_{2} w_{2}\right)$.

The following lemma is due to Jakobsen and Kac [1989].

Lemma 4.1. Let $z_{1}, z_{2}, \ldots, z_{N}, w_{1}, w_{2}, \ldots, w_{N} \in \mathbb{C}_{q}\left[s^{ \pm 1}, t^{ \pm 1}\right]$

$$
\left(\begin{array}{cc}
0 & z_{1} \\
0 & 0
\end{array}\right)\left(\begin{array}{cc}
0 & z_{2} \\
0 & 0
\end{array}\right) \cdots\left(\begin{array}{cc}
0 & z_{N} \\
0 & 0
\end{array}\right)\left(\begin{array}{cc}
0 & 0 \\
w_{1} & 0
\end{array}\right)\left(\begin{array}{cc}
0 & 0 \\
w_{2} & 0
\end{array}\right) \cdots\left(\begin{array}{cc}
0 & 0 \\
w_{N} & 0
\end{array}\right) \cdot 1
$$

$=\sum_{s=1}^{N} \sum_{\gamma \in \operatorname{Par}_{s}(N)\left[\pi_{1} \times \pi_{2}\right] \in\left(S_{N} \times S_{N}\right)(\gamma)}(-1)^{\gamma_{1}-1}(-\mu) \kappa\left(z_{\pi_{1}(1)} w_{\pi_{2}(1)} \cdots z_{\pi_{1}\left(\gamma_{1}\right)} w_{\pi_{2}\left(\gamma_{1}\right)}\right)$

$$
\begin{array}{r}
\cdot(-1)^{\gamma_{2}-1}(-\mu) \kappa\left(z_{\pi_{1}\left(\gamma_{1}+1\right)} w_{\pi_{2}\left(\gamma_{1}+1\right)} \cdots z_{\pi_{1}\left(\gamma_{2}\right)} w_{\pi_{2}\left(\gamma_{2}\right)}\right) \cdot \\
\cdots(-1)^{\gamma_{s}-1}(-\mu) \kappa\left(z_{\pi_{1}\left(\gamma_{1}+\cdots+\gamma_{s-1}+1\right)} w_{\pi_{2}\left(\gamma_{1}+\cdots+\gamma_{s-1}+1\right)} \cdots z_{\pi_{1}(N)} w_{\pi_{2}(N)}\right) \cdot 1
\end{array}
$$

Lemma 4.2. Let $a_{i}, c_{i}, b_{j}, d_{j} \in \mathbb{C}_{q}$ for $i=1, \ldots, m$ and $j=1, \ldots, n$. Let $R=$ $\left(a_{i} c_{j}\right)_{m \times m}$ and $U=\left(b_{i} d_{j}\right)_{n \times n}$, and set

$$
\Lambda=\left(\begin{array}{cc}
R & 0 \\
0 & U
\end{array}\right)_{(m+n) \times(m+n)}=\left(\lambda_{i, j}\right)_{(m+n) \times(m+n)} .
$$

Then

(4-3) $\quad E_{21}\left(a_{1}\right) \cdots E_{21}\left(a_{m}\right) E_{23}\left(b_{1}\right) \cdots E_{23}\left(b_{n}\right) E_{12}\left(c_{1}\right) \cdots$

$$
E_{12}\left(c_{m}\right) E_{32}\left(d_{1}\right) \cdots E_{32}\left(d_{n}\right) \cdot 1
$$

$$
\begin{array}{r}
\sum_{s=1}^{m+n} \sum_{\gamma \in \operatorname{Par}_{s}(m+n)\left[\pi_{1} \times \pi_{2}\right] \in\left(S_{m+n} \times S_{m+n}\right)(\gamma)}(-1)^{\gamma_{1}-1}(-\mu) \kappa\left(\lambda_{\pi_{1}(1), \pi_{2}(1)} \cdots \lambda_{\pi_{1}\left(\gamma_{1}\right) \pi_{2}\left(\gamma_{1}\right)}\right) \\
\cdot(-1)^{\gamma_{2}-1}(-\mu) \kappa\left(\lambda_{\pi_{1}\left(\gamma_{1}+1\right), \pi_{2}\left(\gamma_{1}+1\right)} \cdots \lambda_{\pi_{1}\left(\gamma_{2}\right), \pi_{2}\left(\gamma_{2}\right)}\right) \\
\cdots(-1)^{\gamma_{s}-1}(-\mu) \kappa\left(\lambda_{\pi_{1}\left(\gamma_{1}+\cdots \gamma_{s-1}+1\right), \pi_{2}\left(\gamma_{1}+\cdots+\gamma_{s-1}+1\right)} \cdots \lambda_{\pi_{1}(N), \pi_{2}(N)}\right) \cdot 1 .
\end{array}
$$

Remark 4.3. It is easy to see that $\lambda_{i, j}$ in every summand should be from different rows and different columns of $\Lambda$. And if the summand of (4-3) contains some $\lambda_{i, j}=0$, then this summand is 0 . Hence (4-3) is in fact the sum of those $\lambda_{i, j}$ from $R$ and $U$.

Proof. We proceed by induction on $n$. For $n=0$, (4-3) is just (4-2). Next assume (4-3) is true up to $n-1$,

$$
E_{21}\left(a_{1}\right) \cdots E_{21}\left(a_{m}\right) E_{23}\left(b_{1}\right) \cdots E_{23}\left(b_{n}\right) E_{12}\left(c_{1}\right) \cdots E_{12}\left(c_{m}\right) E_{32}\left(d_{1}\right) \cdots E_{32}\left(d_{n}\right) \cdot 1
$$




$$
\begin{aligned}
& =E_{21}\left(a_{1}\right) \cdots E_{21}\left(a_{m}\right) E_{23}\left(b_{1}\right) \cdots E_{23}\left(b_{n-1}\right) \\
& \cdot\left(E_{12}\left(c_{1}\right) E_{23}\left(b_{n}\right)-E_{13}\left(c_{1} b_{n}\right)\right) E_{12}\left(c_{2}\right) \cdots E_{12}\left(c_{m}\right) E_{32}\left(d_{1}\right) \cdots E_{32}\left(d_{n}\right) \cdot 1 \\
& =E_{21}\left(a_{1}\right) \cdots E_{21}\left(a_{m}\right) E_{23}\left(b_{1}\right) \cdots E_{23}\left(b_{n-1}\right) E_{12}\left(c_{1}\right) E_{23}\left(b_{n}\right) E_{12}\left(c_{2}\right) \\
& \cdots E_{12}\left(c_{m}\right) E_{32}\left(d_{1}\right) \cdots E_{32}\left(d_{n}\right) \cdot 1 \\
& -E_{21}\left(a_{1}\right) \cdots E_{21}\left(a_{m}\right) E_{23}\left(b_{1}\right) \cdots E_{23}\left(b_{n-1}\right) E_{12}\left(c_{1}\right) \cdots E_{12}\left(c_{m}\right)\left(E_{12}\left(c_{1} b_{n} d_{1}\right)\right. \\
& \left.+E_{32}\left(d_{1}\right) E_{13}\left(c_{1} b_{n}\right)\right) E_{32}\left(d_{2}\right) \cdots E_{32}\left(d_{n}\right) \cdot 1 \\
& =E_{21}\left(a_{1}\right) \cdots E_{21}\left(a_{m}\right) E_{23}\left(b_{1}\right) \cdots E_{23}\left(b_{n-1}\right) E_{12}\left(c_{1}\right) E_{23}\left(b_{n}\right) E_{12}\left(c_{2}\right) \\
& \cdots E_{12}\left(c_{m}\right) E_{32}\left(d_{1}\right) \cdots E_{32}\left(d_{n}\right) \cdot 1 \\
& +\sum_{i=1}^{n} E_{21}\left(a_{1}\right) \cdots E_{21}\left(a_{m}\right) E_{23}\left(b_{1}\right) \cdots E_{23}\left(b_{n-1}\right) \\
& \left.\cdot E_{12}\left(-c_{1} b_{n} d_{i}\right) E_{12}\left(c_{2}\right) \cdots E_{12}\left(c_{m}\right) E_{32}\left(d_{1}\right) \cdots \widehat{E_{32}\left(d_{i}\right.}\right) \cdots E_{32}\left(d_{n}\right) \cdot 1 \\
& =E_{21}\left(a_{1}\right) \cdots E_{21}\left(a_{m}\right) E_{23}\left(b_{1}\right) \cdots E_{23}\left(b_{n-1}\right) E_{12}\left(c_{1}\right) E_{12}\left(c_{2}\right) \\
& \cdots E_{12}\left(c_{m}\right) E_{23}\left(b_{n}\right) E_{32}\left(d_{1}\right) \cdots E_{32}\left(d_{n}\right) \cdot 1 \\
& +\sum_{i=1}^{n} \sum_{j=1}^{m} E_{21}\left(a_{1}\right) \cdots E_{21}\left(a_{m}\right) E_{23}\left(b_{1}\right) \cdots E_{23}\left(b_{n-1}\right) \\
& \left.\cdot E_{12}\left(c_{1}\right) \cdots E_{12}\left(-c_{j} b_{n} d_{i}\right) \cdots E_{12}\left(c_{m}\right) E_{32}\left(d_{1}\right) \cdots \widehat{E_{32}\left(d_{i}\right.}\right) \cdots E_{32}\left(d_{n}\right) \cdot 1 \\
& =\sum_{i=1}^{n} \sum_{j>i} E_{21}\left(a_{1}\right) \cdots E_{21}\left(a_{m}\right) E_{23}\left(b_{1}\right) \cdots E_{23}\left(b_{n-1}\right) E_{12}\left(c_{1}\right) E_{12}\left(c_{2}\right) \cdots E_{12}\left(c_{m}\right) \\
& \left.\cdot E_{32}\left(d_{1}\right) \cdots \widehat{E_{32}\left(d_{i}\right.}\right) \cdots E_{32}\left(d_{j-1}\right) E_{32}\left(-d_{i} b_{n} d_{j}-d_{j} b_{n} d_{i}\right) \cdots E_{32}\left(d_{n}\right) \cdot 1 \\
& +\sum_{i=1}^{n} E_{21}\left(a_{1}\right) \cdots E_{21}\left(a_{m}\right) E_{23}\left(b_{1}\right) \cdots E_{23}\left(b_{n-1}\right) \\
& \left.\cdot E_{12}\left(c_{1}\right) E_{12}\left(c_{2}\right) \cdots E_{12}\left(c_{m}\right) E_{32}\left(d_{1}\right) \cdots \widehat{E_{32}\left(d_{i}\right.}\right) \cdots E_{32}\left(d_{n}\right)(-\mu) \kappa\left(b_{n} d_{i}\right) \cdot 1 \\
& +\sum_{i=1}^{n} \sum_{j=1}^{m} E_{21}\left(a_{1}\right) \cdots E_{21}\left(a_{m}\right) E_{23}\left(b_{1}\right) \cdots E_{23}\left(b_{n-1}\right) \\
& \left.\cdot E_{12}\left(c_{1}\right) \cdots E_{12}\left(-c_{j} b_{n} d_{i}\right) \cdots E_{12}\left(c_{m}\right) E_{32}\left(d_{1}\right) \cdots \widehat{E_{32}\left(d_{i}\right.}\right) \cdots E_{32}\left(d_{n}\right) \cdot 1 \text {. }
\end{aligned}
$$

Because (4-3) is true for $n-1$, we expand to see it is also true for $n$.

Lemma 4.4. The levels are orthogonal with respect to the the hermitian form; that is, the form vanishes when applied to two vectors from different levels.

Proof. Only need to prove those elements in the basis $\mathscr{B}$. Let

$$
\begin{aligned}
& u=E_{12}\left(a_{1}\right) \cdots E_{12}\left(a_{m}\right) E_{32}\left(b_{1}\right) \cdots E_{32}\left(b_{n}\right) \cdot 1 \\
& v=E_{12}\left(c_{1}\right) \cdots E_{12}\left(c_{k}\right) E_{32}\left(d_{1}\right) \cdots E_{32}\left(d_{l}\right) \cdot 1
\end{aligned}
$$

and suppose $(m, n) \neq(k, l)$. 
First we prove $(u, v)=0$ with $m=0$. If $k=0$, then, supposing $n>l$, we have

$$
\begin{aligned}
(u, v) & =\left(E_{32}\left(b_{1}\right) \cdots E_{32}\left(b_{n}\right) \cdot 1, E_{32}\left(d_{1}\right) \cdots E_{32}\left(d_{l}\right) \cdot 1\right) \\
& =\left((-1)^{l} E_{23}\left(\bar{d}_{l}\right) \cdots E_{23}\left(\bar{d}_{1}\right) E_{32}\left(b_{1}\right) \cdots E_{32}\left(b_{n}\right) \cdot 1,1\right) .
\end{aligned}
$$

Then, by Lemma $3.3, \operatorname{lev}\left(E_{23}\left(\bar{d}_{l}\right) \cdots E_{23}\left(\bar{d}_{1}\right) E_{32}\left(b_{1}\right) \cdots E_{32}\left(b_{n}\right) \cdot 1\right)=(0, n-l)$, or, if $E_{23}\left(\bar{d}_{l}\right) \cdots E_{23}\left(\bar{d}_{1}\right) E_{32}\left(b_{1}\right) \cdots E_{32}\left(b_{n}\right) \cdot 1=0$, then $(u, v)=0$.

For $k>0$,

$$
\begin{aligned}
(u, v)= & \left(E_{32}\left(b_{1}\right) \cdots E_{32}\left(b_{n}\right) \cdot 1, E_{12}\left(c_{1}\right) \cdots E_{12}\left(c_{k}\right) E_{32}\left(d_{1}\right) \cdots E_{32}\left(d_{l}\right) \cdot 1\right) \\
= & \left(-E_{21}\left(\overline{c_{1}}\right) E_{32}\left(b_{1}\right) \cdots E_{32}\left(b_{n}\right) \cdot 1,\right. \\
& \left.E_{12}\left(c_{2}\right) \cdots E_{12}\left(c_{k}\right) E_{32}\left(d_{1}\right) \cdots E_{32}\left(d_{l}\right) \cdot 1\right) .
\end{aligned}
$$

Then from Lemma 3.3, we have $-E_{21}\left(\overline{c_{1}}\right) E_{32}\left(b_{1}\right) \cdots E_{32}\left(b_{n}\right) \cdot 1=0$, and $(u, v)=0$.

Without loss of generality, we can assume that $m \leq k$; then

$$
\begin{aligned}
(u, v)= & \left(E_{12}\left(a_{1}\right) \cdots E_{12}\left(a_{m}\right) E_{32}\left(b_{1}\right) \cdots E_{32}\left(b_{n}\right) \cdot 1,\right. \\
& \left.E_{12}\left(c_{1}\right) \cdots E_{12}\left(c_{k}\right) E_{32}\left(d_{1}\right) \cdots E_{32}\left(d_{l}\right) \cdot 1\right) \\
= & \left(E_{32}\left(b_{1}\right) \cdots E_{32}\left(b_{n}\right) \cdot 1,\right. \\
& \left.(-1)^{m} E_{21}\left(\overline{a_{m}}\right) \cdots E_{21}\left(\overline{a_{1}}\right) \cdot E_{12}\left(c_{1}\right) \cdots E_{12}\left(c_{k}\right) E_{32}\left(d_{1}\right) \cdots E_{32}\left(d_{l}\right) \cdot 1\right) .
\end{aligned}
$$

From Lemma 3.3,

$$
\operatorname{lev}\left(E_{21}\left(\overline{a_{m}}\right) \cdots E_{21}\left(\overline{a_{1}}\right) \cdot E_{12}\left(c_{1}\right) \cdots E_{12}\left(c_{k}\right) E_{32}\left(d_{1}\right) \cdots E_{32}\left(d_{l}\right) \cdot 1\right)=(k-m, n)
$$

or

$$
E_{21}\left(\overline{a_{m}}\right) \cdots E_{21}\left(\overline{a_{1}}\right) \cdot E_{12}\left(c_{1}\right) \cdots E_{12}\left(c_{k}\right) E_{32}\left(d_{1}\right) \cdots E_{32}\left(d_{l}\right) \cdot 1=0 .
$$

Then, going back to the case $m=0$, we get $(u, v)=0$.

Similarly to [Gao and Zeng 2006, Proposition 4.11] and together with Lemma 4.2, we have

Proposition 4.5. The hermitian form on the element $h$ in level $(m, n)$ is a polynomial in $\mu$, with leading term $c(-1)^{m+n}(-\mu)^{m+n}=c \mu^{m+n}$ for some constant $c>0$.

Theorem 4.6. $(\pi, V)$ can be made unitary if and only if $\mu>0$.

Proof. From [Gao and Zeng 2006, Theorem 4.12], the hermitian form in level $(0, n)$ and $(m, 0)$ is positive definite if and only if $\mu>0$.

Define

$$
T_{a, b}\left(s^{m_{1}} t^{n_{1}} s^{m_{2}} t^{n_{2}} \cdots s^{m_{k}} t^{n_{k}}\right)=s^{m_{1}+a} t^{n_{1}+b} s^{m_{2}+a} t^{n_{2}+b} \cdots s^{m_{k}+a} t^{n_{k}+b}
$$


for $a, b \in \mathbb{Z}$. Extend this operator to the linear operator $\widetilde{T_{a, b}}$ on $V$ by

$$
\begin{aligned}
& \widetilde{T_{a, b}}\left(E_{12}\left(\alpha_{1}\right) E_{12}\left(\alpha_{2}\right) \cdots E_{12}\left(\alpha_{k}\right) E_{32}\left(\beta_{1}\right) E_{32}\left(\beta_{2}\right) \cdots E_{32}\left(\beta_{l}\right) \cdot 1\right) \\
& =E_{12}\left(T_{a, b} \alpha_{1}\right) E_{12}\left(T_{a, b} \alpha_{2}\right) \cdots E_{12}\left(T_{a, b} \alpha_{k}\right) \\
& \cdot E_{32}\left(T_{a, b} \beta_{1}\right) E_{32}\left(T_{a, b} \beta_{2}\right) \cdots E_{32}\left(T_{a, b} \beta_{l}\right) \cdot 1 .
\end{aligned}
$$

Following Lemma 4.2, $\widetilde{T_{a, b}}$ preserves the hermitian form on $V$. Denote

$$
L_{l, r}(M, N)=\operatorname{span}\left\{E_{12}\left(s^{m_{1}} t^{n_{1}}\right) \cdots E_{12}\left(s^{m_{l}} t^{n_{l}}\right) E_{32}\left(s^{j_{1}} t^{k_{1}}\right) \cdots E_{32}\left(s^{j_{r}} t^{k_{r}}\right) \cdot 1\right.
$$

for $\mid m_{i}, n_{i} \geqq 0$ and $i=1, \ldots, l$, with $j_{\iota}, k_{\iota} \geqq 0$,

$$
\left.\sum_{i=1}^{l} m_{i}+\sum_{\iota=1}^{r} j_{\iota} \leqq M, \quad \text { and } \quad \sum_{i=1}^{r} n_{i}+\sum_{\iota=1}^{r} k_{\iota} \leqq N\right\} .
$$

Since the hermitian form on two different levels is 0 , we will prove the unitarity by induction on the level.

For any $\mu>0$, the form is definite in level $(0, n)$; see [Gao and Zeng 2006, Theorem 4.12]. Suppose it is definite in level $(r, n)$ for those $r<m$ and it is not definite in level $(m, n)$.

From Proposition 4.5, we know that the hermitian form restricted to this level should be positive definite for $\mu$ big enough. Assuming it is not positive definite for some $\mu>0$, there exist $M, N$ such that the form restricted to $L_{m, n}(M, N)$ is not positive definite. From Proposition 4.5, the form on $L_{l, r}(M, N)$ varies smoothly with $\mu$. Then we can find a $\mu_{0}$ for which the form is not positive definite and, for all $\mu>\mu_{0}$, it is positive definite. We write $(\cdot, \cdot)_{\mu}$ for the hermitian form at $\mu$.

Thus the radical of the form is nontrivial at $\mu_{0}$, that is, there exists a nonzero $\widetilde{h} \in L_{m, n}(M, N)$ such that, for any $h \in L_{m, n}(M, N)$, we have

$$
\widetilde{h}, h)_{\mu_{0}}=0 \text {. }
$$

Therefore for any arbitrary element $h_{m-1, n}$ in $L_{m-1, n}(M, N)$ and any $c \in \mathbb{C}$, we have

$$
\left(E_{21}(c) \cdot \widetilde{h}, h_{m-1, n}\right)_{\mu_{0}}=0 .
$$

Since the form is positive definite in level $(m-1, n)$, we have $E_{21}(c) \cdot \widetilde{h}=0$ for any $c \in \mathbb{C}$. Replacing $\widetilde{h}$ by $\widetilde{T_{-a,-b}}(\tilde{h})$ if necessary, we can write

$$
\widetilde{h}=\sum_{i=1}^{m} a_{i}\left(E_{12}(1)\right)^{i} x_{i}
$$

where $x_{i}=\sum E_{12}\left(\alpha_{i+1}\right) \cdots E_{12}\left(\alpha_{m}\right) E_{32}\left(\beta_{1}\right) \cdots E_{32}\left(\beta_{n}\right) \cdot 1$ (here it is a finite sum), $\alpha_{i}, \beta_{j}$ is the of form $s^{l} t^{k}$, and $l, k$ cannot both be 0 .

Let $i_{0}$ be the smallest index such that $a_{i_{0}} \neq 0$; then $i_{0} \geq 1$. 


\section{Since}

$$
\begin{aligned}
E_{21}(c) & \left(E_{12}(1)\right)^{i} E_{12}\left(\alpha_{i+1}\right) \cdots E_{12}\left(\alpha_{m}\right) E_{32}\left(\beta_{1}\right) \cdots E_{32}\left(\beta_{n}\right) \cdot 1 \\
= & \left(E_{12}(1)\right)^{i} E_{21}(c) E_{12}\left(\alpha_{i+1}\right) \cdots E_{12}\left(\alpha_{m}\right) E_{32}\left(\beta_{1}\right) \cdots E_{32}\left(\beta_{n}\right) \cdot 1 \\
& +i\left(E_{12}(1)\right)^{i-1}\left(E_{22}(c)-E_{11}(c)\right) E_{12}\left(\alpha_{i+1}\right) \cdots E_{12}\left(\alpha_{m}\right) E_{32}\left(\beta_{1}\right) \cdots E_{32}\left(\beta_{n}\right) \cdot 1 \\
& +(-2 c) \frac{i(i-1)}{2}\left(E_{12}(1)\right)^{i-1} E_{12}\left(\alpha_{i+1}\right) \cdots E_{12}\left(\alpha_{m}\right) E_{32}\left(\beta_{1}\right) \cdots E_{32}\left(\beta_{n}\right) \cdot 1 \\
= & \left(E_{12}(1)\right)^{i} E_{21}(c) E_{12}\left(\alpha_{i+1}\right) \cdots E_{12}\left(\alpha_{m}\right) E_{32}\left(\beta_{1}\right) \cdots E_{32}\left(\beta_{n}\right) \cdot 1 \\
& +i\left(E_{12}(1)\right)^{i-1}((-2 c)(m-i)-n) E_{12}\left(\alpha_{i+1}\right) \cdots E_{12}\left(\alpha_{m}\right) E_{32}\left(\beta_{1}\right) \cdots E_{32}\left(\beta_{n}\right) \cdot 1 \\
& +i\left(E_{12}(1)\right)^{i-1} E_{12}\left(\alpha_{i+1}\right) \cdots E_{12}\left(\alpha_{m}\right) E_{32}\left(\beta_{1}\right) \cdots E_{32}\left(\beta_{n}\right)\left(E_{22}(c)-E_{11}(c)\right) \cdot 1 \\
& +(-2 c) \frac{i(i-1)}{2}\left(E_{12}(1)\right)^{i-1} E_{12}\left(\alpha_{i+1}\right) \cdots E_{12}\left(\alpha_{m}\right) E_{32}\left(\beta_{1}\right) \cdots E_{32}\left(\beta_{n}\right) \cdot 1 \\
= & \left(E_{12}(1)\right)^{i} E_{21}(c) E_{12}\left(\alpha_{i+1}\right) \cdots E_{12}\left(\alpha_{m}\right) E_{32}\left(\beta_{1}\right) \cdots E_{32}\left(\beta_{n}\right) \cdot 1 \\
& +\left[i c\left(-\mu_{0}\right)+i((-2 c)(m-i)-n)+(-2 c) \frac{i(i-1)}{2}\right] . \\
& \left(E_{12}(1)\right)^{i-1} E_{12}\left(\alpha_{i+1}\right) \cdots E_{12}\left(\alpha_{m}\right) E_{32}\left(\beta_{1}\right) \cdots E_{32}\left(\beta_{n}\right) \cdot 1,
\end{aligned}
$$

we have

$$
E_{21}(c) \tilde{h}=\gamma a_{i_{0}}\left(E_{12}(1)\right)^{i_{0}-1} x_{i_{0}}+R,
$$

where $R$ contains those terms with powers of $E_{12}(1)$ greater than $i_{0}-1$ and

$$
\begin{aligned}
\gamma & =i_{0} c\left(-\mu_{0}\right)+i_{0}\left((-2 c)\left(m-i_{0}\right)-n\right)+(-2 c) \frac{i_{0}\left(i_{0}-1\right)}{2} \\
& =c i_{0}\left(-\mu_{0}-\left(m-i_{0}\right)-(m-1)\right) .
\end{aligned}
$$

Since $m \geq i_{0} \geq 1, \mu_{0} \geq 0$, and $\gamma \neq 0$, this contradicts $E_{21}(c) \widetilde{h}=0$. Thus, for any $\mu>0$, the hermitian form is positive definite.

\section{Acknowledgments}

I am grateful to my supervisors Professors Nantel Bergeron and Yun Gao for their encouragement and support during the preparation of this paper. I am especially grateful to Professor Yun Gao for drawing my attention to this subject.

\section{References}

[Allison et al. 1997] B. N. Allison, S. Azam, S. Berman, Y. Gao, and A. Pianzola, "Extended affine Lie algebras and their root systems", Mem. Amer. Math. Soc. 126:603 (1997), x+122. MR 97i:17015 Zbl 0879.17012

[Baranovsky et al. 2000] V. Baranovsky, S. Evens, and G. V., "Representations of quantum tori and double-affine Hecke algebras", preprint, 2000. math.RT/0005024

[Berman and Szmigielski 1999] S. Berman and J. Szmigielski, "Principal realization for the extended affine Lie algebra of type $\mathrm{sl}_{2}$ with coordinates in a simple quantum torus with two generators", pp. 39-67 in Recent developments in quantum affine algebras and related topics (Raleigh, 
NC, 1998), edited by N. Jing and K. C. Misra, Contemp. Math. 248, Amer. Math. Soc., Providence, RI, 1999. MR 2001h:17038 Zbl 1031.17014

[Berman et al. 1996] S. Berman, Y. Gao, and Y. S. Krylyuk, "Quantum tori and the structure of elliptic quasi-simple Lie algebras", J. Funct. Anal. 135:2 (1996), 339-389. MR 97b:17007 Zbl 0847.17009

[Billig and Zhao 2004] Y. Billig and K. Zhao, "Vertex operator representations of quantum tori at roots of unity”, Comm. Contemp. Math. 6:1 (2004), 195-220. MR 2005b:17054 Zbl pre02078551

[Eswara Rao 2003] S. Eswara Rao, "Unitary modules for EALAs co-ordinatized by a quantum torus", Comm. Algebra 31:5 (2003), 2245-2256. MR 2004e:17024 Zbl 1106.17029

[Eswara Rao 2004] S. Eswara Rao, "A class of integrable modules for the core of EALA coordinatized by quantum tori”, J. Algebra 275:1 (2004), 59-74. MR 2005a:17024 Zbl pre02105787

[Eswara Rao and Batra 2002] S. Eswara Rao and P. Batra, "A new class of representations of EALA coordinated by quantum tori in two variables", Canad. Math. Bull. 45:4 (2002), 672-685. MR 2003k:17031 Zbl 1034.17015

[Eswara Rao and Zhao 2004] S. Eswara Rao and K. Zhao, "Highest weight irreducible representations of rank 2 quantum tori”, Math. Res. Lett. 11:5-6 (2004), 615-628. MR 2005j:17006 Zbl 1094.17011

[Etingof et al. 1998] P. I. Etingof, I. B. Frenkel, and A. A. Kirillov, Jr., Lectures on representation theory and Knizhnik-Zamolodchikov equations, Mathematical Surveys and Monographs 58, American Mathematical Society, Providence, RI, 1998. MR 2001b:32028 Zbl 0903.17006

[Fĕgin and Frenkel 1990] B. L. Fer̆gin and E. V. Frenkel, "Affine Kac-Moody algebras and semiinfinite flag manifolds", Comm. Math. Phys. 128 (1990), 161-189. MR 92f:17026 Zbl 0722.17019

[Gao 2000a] Y. Gao, "Representations of extended affine Lie algebras coordinatized by certain quantum tori”, Compositio Math. 123:1 (2000), 1-25. MR 2002c:17036 Zbl 0958.17008

[Gao 2000b] Y. Gao, "Vertex operators arising from the homogeneous realization for $\widehat{\mathrm{gl}}_{N}$ ", Comm. Math. Phys. 211:3 (2000), 745-777. MR 2001k:17044 Zbl 0982.17012

[Gao 2002] Y. Gao, "Fermionic and bosonic representations of the extended affine Lie algebra $\mathfrak{g l}_{N}\left(\mathbb{C}_{q}\right)$ ", Canad. Math. Bull. 45:4 (2002), 623-633. MR 2003h:17030 Zbl 1040.17017

[Gao and Zeng 2006] Y. Gao and Z. Zeng, "Hermitian representations of the extended affine Lie algebra $\mathfrak{g l}_{2}\left(\mathbb{C}_{q}\right)$ ", Adv. Math. 207:1 (2006), 244-265. MR 2007g:17023 Zbl 1106.17030

[Golenishcheva-Kutuzova and Kac 1998] M. I. Golenishcheva-Kutuzova and V. G. Kac, "Г-conformal algebras”, J. Math. Phys. 39:4 (1998), 2290-2305. MR 99d:81048 Zbl 1031.81527

[Høegh-Krohn and Torrésani 1990] R. Høegh-Krohn and B. Torrésani, "Classification and construction of quasisimple Lie algebras", J. Funct. Anal. 89:1 (1990), 106-136. MR 91a:17008 Zbl 0792.17019

[Jakobsen and Kac 1989] H. P. Jakobsen and V. Kac, "A new class of unitarizable highest weight representations of infinite-dimensional Lie algebras. II”, J. Funct. Anal. 82:1 (1989), 69-90. MR 89m:17032 Zbl 0688.17007

[Lau 2005] M. Lau, "Bosonic and fermionic representations of Lie algebra central extensions", $A d v$. Math. 194:2 (2005), 225-245. MR 2005m:17008 Zbl pre02183827

[Lin and Tan 2004] W. Lin and S. Tan, "Representations of the Lie algebra of derivations for quantum torus", J. Algebra 275:1 (2004), 250-274. MR 2005a:17014 Zbl 1106.17020

[Lin and Tan 2006] W. Lin and S. Tan, "Harish-Chandra modules for the $q$-analog Virasoro-like algebra”, J. Algebra 297:1 (2006), 254-272. MR 2007a:17040 Zbl pre05028847 
[Manin 1991] Y. I. Manin, Topics in noncommutative geometry, M. B. Porter Lectures, Princeton University Press, Princeton, NJ, 1991. MR 92k:58024 Zbl 0724.17007

[Miki 2004] K. Miki, "Integrable irreducible highest weight modules for $\operatorname{sl}_{2}\left(\mathbf{C}_{p}\left[x^{ \pm 1}, y^{ \pm 1}\right]\right)$ ", Osaka J. Math. 41:2 (2004), 295-326. MR 2005d:17024 Zbl 02111459

[Su and Zhu 2005] Y. Su and L. Zhu, "Representations of the derivation algebra of the localization of the quantum plane at $q=-1$ ”, Comm. Algebra 33:12 (2005), 4375-4382. MR 2006m:17007 Zbl pre02248208

[Varagnolo and Vasserot 1998] M. Varagnolo and E. Vasserot, "Double-loop algebras and the Fock space”, Invent. Math. 133:1 (1998), 133-159. MR 99g:17035 Zbl 0904.17014

[Wakimoto 1985] M. Wakimoto, "Extended affine Lie algebras and a certain series of Hermitian representations", manuscript, 1985.

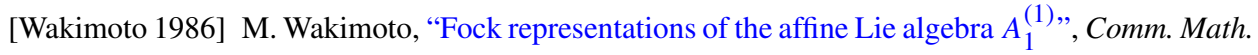
Phys. 104:4 (1986), 605-609. MR 87m:17011 Zbl 0587.17009

[Zhang and Zhao 1996] H. Zhang and K. Zhao, "Representations of the Virasoro-like algebra and its $q$-analog”, Comm. Algebra 24:14 (1996), 4361-4372. MR 97m:17031 Zbl 0891.17016

Received October 2, 2006. Revised November 12, 2006.

\section{ZITING ZENG}

SCHOOL OF MATHEMATICAL SCIENCES

BEIJING NORMAL UNIVERSITY

100875 BEIJING

CHINA

zengzt@bnu.edu.cn 